\title{
Dissociating Inherent Emotional and \\ Associated Motivational Salience in Human Face Processing
}

Dissertation

Zur Erlangung des mathematisch-naturwissenschaftlichen Doktorgrades

"Doctor rerum naturalium"

der Georg-August-Universität Göttingen

im Promotionsprogramm Behavior and Cognition (BeCog)

der Georg-August University School of Science (GAUSS)

vorgelegt von

\section{Wiebke Hammerschmidt}

aus Bergisch Gladbach

Göttingen, Januar 2018 


\section{Betreuungsausschuss}

Prof. Dr. Annekathrin Schacht

Affektive Neurowissenschaft und Psychophysiologie, Institut für Psycholgie, Universität Göttingen

Prof. Dr. Julia Fischer

Kognitive Ethologie, Deutsches Primatenzentrum, Göttingen

Dr. Igor Kagan

Decision and Awareness Group/Kognitive Neurowissenschaften, Deutsches

Primatenzentrum, Göttingen

\section{Mitglieder der Prüfungskommision}

Referent/in: Prof. Dr. Annekathrin Schacht

Affektive Neurowissenschaft und Psychophysiologie, Institut für Psycholgie, Universität Göttingen

Koreferent/in: Dr. Igor Kagan

Decision and Awareness Group/Kognitive Neurowissenschaften, Deutsches

Primatenzentrum, Göttingen

Weitere Mitglieder der Prüfungskommision:

Prof. Dr. Julia Fischer

Kognitive Ethologie, Deutsches Primatenzentrum, Göttingen

Dr. Tanya Behne

Kognitive Entwicklungspsychologie, Institut für Psycholgie, Universität Göttingen

Dr. Arezoo Pooresmaeili

Perception and Cognition Group, European Neurosciene Institute (ENI), Göttingen

Dr. Oliver Schülke

Verhaltensökologie, Johann-Friedrich-Blumenbach Institut für Zoologie and

Anthropologie, Göttingen

Tag der mündlichen Prüfung: 11. April 2018 


"It is our choices, Harry, that show what we truly are, far more than our abilities."

J.K. Rowling

Harry Potter and the Chamber of Secrets 



\begin{abstract}
The human brain is challenged to select only the most relevant stimuli, assured by salienceand goal-driven attention mechanisms. As not only stimuli carrying inherent salience (e.g., facial expressions of emotion), but also learned stimulus-reward associations were shown to elicit attentional prioritization, an additional value-driven attention mechanism (B. A. Anderson, 2013) was suggested. A direct comparison, however, was absent. Therefore, the aim of the present $\mathrm{PhD}$ project was to fill this gap in the literature by investigating the dissociation of neural impacts of associated motivational and inherent emotional salience on human face processing. Three studies, measuring event-related brain potentials (ERPs), were conducted to examine the potential processing advantage of neutral expressions previously associated with motivational salience via explicit (Study 1) and implicit (Study 2) associative learning. In addition, ERP modulations elicited by associated motivational salience were compared to those evoked by facial expressions of emotion. Study 3 aimed to assess the integration of inherent emotional and associative motivational salience. The results demonstrated that inherently neutral stimuli can acquire increased salience via associative learning and might impact initial perceptual (Study 1) or elaborative processing stages (Study 2 and 3). The sensory encoding, however, was limited to biologically determined sources of emotional salience (Study 1 to 3 ) as provided by facial expressions of emotion. In summary, the findings confirmed that emotionally and motivationally relevant stimuli elicit a comparable attentional prioritization, as proposed by the value-driven attention mechanism. Associated motivational salience, however, does not seem to trigger an inherent emotional meaning.
\end{abstract}

Keywords: Event-related brain potentials (ERPs), Motivational salience, Emotional expressions, Associative learning. 



\section{Zusammenfassung}

Das menschliche Gehirn steht vor der Herausforderung nur die relevantesten Reize zu selektieren, was durch salienz- und zielgetriebene Aufmerksamkeitsprozesse sichergestellt wird. Da aber nicht nur Stimuli, denen eine inhärente Salienz inne liegt (z.B. emotionale Gesichtsausdrücke), sondern auch gelernte Stimulus-Belohnungs-Assoziationen eine bevorzugte Aufmerksamkeitszuteilung hervorrufen, wurde die Existenz eines weiteren, wertigkeitsgetriebenen Aufmerksamkeitsprozesses (B. A. Anderson, 2013) vorgeschlagen. Das Ziel des vorliegenden Dissertationsprojekts war die Prüfung einer Abgrenzung von neuralen Auswirkungen von assoziierter motivationaler und inhärent emotionaler Salienz in der menschlichen Gesichterverarbeitung, da ein direkter Vergleich dieser bisher fehlt. Drei Studien, in denen ereignis-korrelierte Hirnpotentiale (EKPs) gemessen wurden, wurden durchgeführt, um diese Lücke in der bisherigen Literatur zu füllen. Ein möglicher Verarbeitungsvorteil von neutralen Gesichtsausdrücken, die vorher durch explizites (Studie 1) und implizites (Studie 2) assoziatives Lernen mit motivationaler Salienz assoziiert wurden, wurde untersucht. Zusätzlich wurden die von assoziierter motivationaler Salienz hervorgerufenen EKP Modulationen, mit denen von inhärent emotionaler Salienz verglichen. Das Ziel von Studie 3 war es, eine mögliche Integration von inhärent emotionaler und assoziierter motivational Salienz zu beurteilen. Die Ergebnisse zeigen, dass inhärent neutrale Stimuli eine verstärkte Salienz durch assoziatives Lernen erlangen können und dass diese die frühe perzeptuelle (Studie 1) oder die spätere ausführlichere Verarbeitung (Studien 2 und 3) beeinflussen können. Eine sensorische Enkodierung war allerdings den biologisch bestimmten Quellen von emotionaler Salienz, den emotionalen Gesichtsausdrücken, vorbehalten (Studien 1 bis 3). Zusammenfassend bestätigen die Ergebnisse, dass emotional und motivational relevante Stimuli ein vergleichbares priorisiertes Verarbeiten hervorrufen können, wie vom wertigkeitsgetriebenen Aufmerksamkeitsprozess vorhergesagt. Allerdings scheint assoziierte motivationale Salienz keinen inhärenten emotionalen Inhalt auszulösen.

Schlagwörter: Ereignis-korrelierte Hirnpotentiale (EKPs), Motivationale Salienz, Emotionale Gesichtsausdrücke, Assoziatives Lernen. 



\section{Contents}

1 General Introduction 1

2 Associated Motivational Salience Impacts Early Sensory Processing of Human Faces

3 Implicit Reward Associations Impact Face Processing: Time-resolved Evidence from Event-related Brain Potentials and Pupil Dilations $\quad 10$

3.1 Introduction . . . . . . . . . . . . . . . . . . . . . . . . . 11

3.2 Materials and Methods . . . . . . . . . . . . . . . . . . . 15

3.3 Results . . . . . . . . . . . . . . . . . . . . . 20

3.4 Discussion . . . . . . . . . . . . . . . . . . . . . . 25

4 Money or Smiles: Independent ERP Effects of Associated Monetary Reward and Happy Faces $\quad 30$

4.1 Introduction . . . . . . . . . . . . . . . . . . . . . . 31

4.2 Materials and Method . . . . . . . . . . . . . . . . . 33

4.3 Results . . . . . . . . . . . . . . . . . . . 36

4.4 Discussion . . . . . . . . . . . . . . . . . . . . . 40

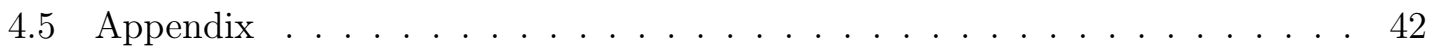

5 General Discussion $\quad 46$

$\begin{array}{ll}\text { References } & 59\end{array}$ 



\section{Chapter 1}

\section{General Introduction}

In our daily life, the human brain is confronted with an overload of visual input and thus challenged to select and integrate only the most relevant information to assure our wellbeing and survival. A preferential processing is, therefore, elicited either by stimuli that are physically distinct or by stimuli that are behaviorally relevant. As humans are social beings, interpreting emotional stimuli (e.g., facial expressions of emotion) are important in social interactions to allow a continuous adaptation of behavior (for a review, see Adolphs, 2003). Emotional stimuli can also be motivationally relevant, as the onset of a happy facial expression might be a reinforcer activating the reward circuitry, whereas an angry face might predict punishment. Thus, the evaluation of a facial stimulus as rewarding or punishing (happy or angry face) might result in a motivational behavior, to approach or to avoid (e.g., Nikitin \& Freund, 2010; Paulus \& Wentura, 2014, 2016). In the course of evolution, to assure a rapid detection of and reaction to both emotionally and motivationally relevant stimuli (for reviews, see Pessoa, 2008; Vuilleumier, 2015), two attention mechanisms were shown to interact with visual perception: a salience-driven and goaldriven attention mechanism (Connor, Egeth, \& Yantis, 2004; Corbetta \& Shulman, 2002; Fecteau \& Munoz, 2006; Theeuwes, 2010, for a review). While salience-driven (bottomup) attention is automatically elicited by distinct physical stimulus features (e.g., a red coat that appears in a crowd of black coats), goal-driven attention is linked to top-down processes guided by selection goals and previous knowledge (e.g., searching for a friend with a red coat in a crowd). However, the debate on the exclusive role of stimulus-driven and goal-driven attention is ongoing and far from settled. Awh, Belopolsky, and Theeuwes (2012) suggested that this dichotomy might not be sufficient, as studies demonstrated an impact of reward history on attention, which can neither be dependent on the physical stimulus salience nor on the participants' goal. Therefore, the authors suggested that both selection and reward history might play an important role on attentional processes. B. A. Anderson (2013) proposed the extension of the conventional dichotomy by suggesting an additional value-driven attention mechanism. To support his conceptual framework, he provided evidence from studies, which employed associative learning and delayed testing tasks (e.g., Della Libera \& Chelazzi, 2009; Raymond \& O'Brien, 2009), showing that learned stimulus-reward associations elicit attentional prioritization, even when the stimuli themselves do not carry inherent salience, if they are task-irrelevant or if the available 
reward is absent. This suggests that also neutral stimuli previously associated with reward gain attentional prioritization, similar to stimuli carrying inherent emotional salience (e.g., facial expressions of emotion).

The well-known motivated attention model of affect (Lang, Bradley, Cuthbert, et al., 1997) provides a theoretical framework, which aims at explaining the prioritized processing of both pleasant and unpleasant stimuli. Lang and colleagues assume that emotions are action dispositions driven by two opponent systems, the appetitive and the aversive system stimulated by reward and punishment. The appetitive or aversive motivation directs the observer's attention and therefore facilitates the perceptual processing of pleasant and unpleasant stimuli (Bradley et al., 2003; Keil et al., 2002). In other words, for Lang, Bradley, Cuthbert, et al. (1997) emotion and motivation are directly linked concepts that play a crucial role in determining human behavior. Integrated in this model are the assumptions of the circumplex model of affect (Russell, 1980, see Figure 1.1) which posits that emotional and motivational aspects elicit a neurophysiological response defined by two dimensions, valence (positive-negative) and arousal (high-low). The arousal reflects the organism's underlying valence evaluation of the stimulus, which, in turn, prompts approach or withdrawal reactions (Posner, Russell, \& Peterson, 2005; Russell, 1980). To explain the similarities of emotion and motivation on a neural level, Pourtois, Schettino, and Vuilleumier (2013) proposed in their Multiple Attention Gain Control (MAGiC) model (see Figure 1.2) that both emotionally and motivationally relevant stimuli can bias visual perception, presumably enabled by an interplay of several brain regions including amongst others the amygdala and the anterior cingulate cortex (ACC). In summary, motivational aspects might be equated with emotional stimulus valence and, thus, they can be seen as a precursor of emotional significance (e.g., Lang \& Bradley, 2010). This supports the existence of a fundamental value-driven attention mechanism (B. A. Anderson, 2013) that is reflected in a facilitated processing of both inherent emotional and motivational salience.

The attentional prioritization elicited by both emotional and motivational factors is often investigated by recordings of event-related brain potentials (ERPs) due to their excellent time resolution, as the neural processing can be traced from early perceptual $(\sim 60-100 \mathrm{~ms}$ after stimulus onset) to later elaborate processing stages (starting $\sim 300 \mathrm{~ms}$ after stimulus onset; Luck, 2014). Stimuli carrying inherent emotional salience are generally known to capture attentional resources (Pessoa, 2008), which holds true for facial expressions of emotion (e.g., Pourtois, Grandjean, Sander, \& Vuilleumier, 2004; Rellecke, Palazova, Sommer, \& Schacht, 2011; Schupp et al., 2004), pictures of affective scenes (e.g., Cuthbert, Schupp, Bradley, Birbaumer, \& Lang, 2000; Olofsson, Nordin, Sequeira, \& Polich, 2008, for a review), or emotional words (Bayer, Sommer, \& Schacht, 2012; Kissler, Herbert, Peyk, \& Junghofer, 2007; Schacht \& Sommer, 2009b). However, in a systematic comparison of the different stimulus domains, it was demonstrated that pictorial stimuli are processed more automated than written words (Bayer \& Schacht, 2014), presumably due to an evolutionary prepared processing of facial expressions of emotion and pictures of affective scenes (Rellecke et al., 2011). Facial stimuli are particularly salient as they automatically attract the observer's attention due to their social significance and biological preparedness (Mehu, 


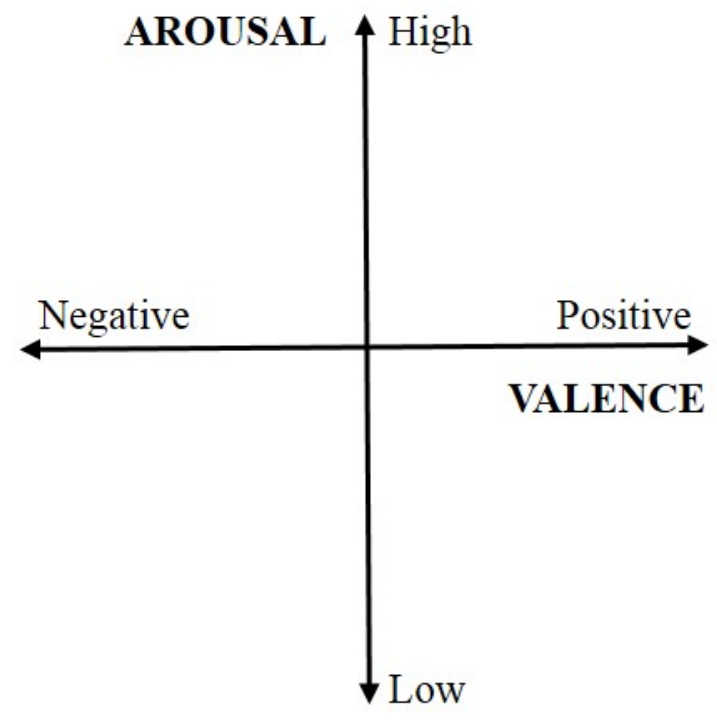

Figure 1.1: The Circumplex Model of Affect [adapted from Russell (1980)].

2014; Öhman, 1986; Vuilleumier, 2005), consistently demonstrated as face-superiority effect (e.g., Crouzet, Kirchner, \& Thorpe, 2010; Langton, Law, Burton, \& Schweinberger, 2008). Compared to neutral facial expressions, faces showing positive (happiness), negative (anger, fear, sadness, disgust) or ambiguous expressions (surprise; e.g., Ekman, 1992) elicit a preferential processing, commonly investigated via event-related brain potentials. Two major ERP components, linked to the preferential processing of emotional stimuli, have been consistently shown to be modulated by facial expressions of emotions, the earlyposterior negativity (EPN) and the late positive complex (LPC), also referred to as late positive potential (LPP). The EPN, a posterior negativity occurring around $200 \mathrm{~ms}$ after stimulus onset, was consistently shown to be modulated by happy, angry or fearful expressions (e.g., Holmes, Nielsen, Tipper, \& Green, 2009; Schacht \& Sommer, 2009a), and linked to an enhanced sensory encoding of emotional stimuli. The LPC, a centro-parietal positivity starting around $300 \mathrm{~ms}$ after stimulus onset and typically lasting for several hundred milliseconds, is linked to higher-order evaluations of emotional stimuli. The LPC is mainly known to be modulated by angry facial expressions, presumably due to an evolutionary threat-related processing bias (Öhman, 1986; Schupp et al., 2004, for more details). The processing advantage of facial expressions of emotion has been, however, outlined to modulate even earlier ERP components. For instance, due to the early occurrence, the $P 1$ component was for a long time considered as solely driven by physical stimulus features, however, it could be demonstrated that threat-related (angry and fearful) expressions elicit enhanced amplitudes on the P1 component (e.g., Pourtois et al., 2004; Rellecke, Sommer, \& Schacht, 2012; Santesso et al., 2008; Vuilleumier \& Pourtois, 2007). The P1 component, an occipital positivity occurring around $100 \mathrm{~ms}$ after stimulus onset, is linked to the activation of the extrastriate visual cortex (Di Russo, Martínez, \& Hillyard, 2003; Di Russo, Martínez, Sereno, Pitzalis, \& Hillyard, 2001) and reflects the early allocation of one's attention to visual stimuli enabling their rapid perceptual processing. Although there is clear evidence that the face-sensitive N170 component, a temporo-occipital negativity 


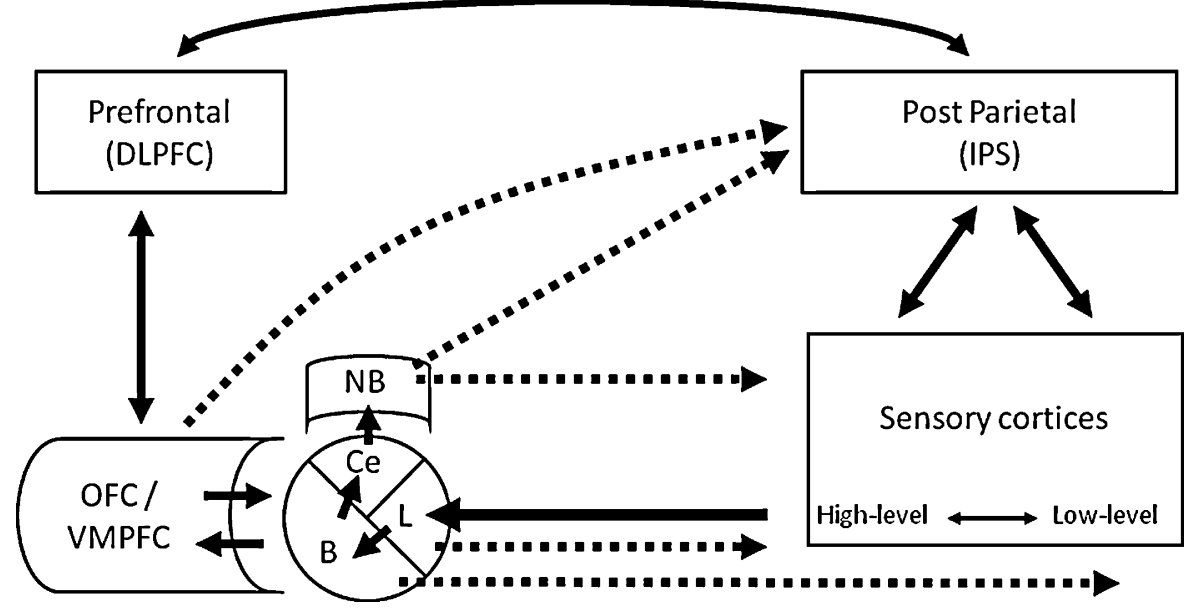

Figure 1.2: The Multiple Attention Gain Control (MAGiC) model (Pourtois et al., 2013). Pourtois and colleagues suggested the amygdala and an interplay of several brain areas to enable the attentional prioritization of both emotionally and motivationally relevant stimuli $(D L P F C=$ dorsolateral prefrontal cortex, $I P S=$ intraparietal sulcus, $O F C=$ orbitofrontal cortex, $V M P F C=$ ventromedial prefrontal cortex, $B=$ basal nucleus, $L=$ lateral nucleus, $C e=$ central nucleus, $N B=$ nucleus basalis).

peaking around $170 \mathrm{~ms}$ after stimulus onset, is related to holistic face processing (e.g., Bentin, Allison, Puce, Perez, \& McCarthy, 1996), the literature on the modulation of the N170 component by facial expressions of emotion is equivocal (for reviews, see Hinojosa, Mercado, \& Carretié, 2015; Rellecke, Sommer, \& Schacht, 2013). To date, there is no clear answer whether configural and emotional features of facial expressions of emotion are processed simultaneously, although an influential model of face recognition suggested an independent processing (Bruce \& Young, 1986).

Furthermore, motivationally, especially reward-related, salient stimuli, either by acquired (learned) associations or by providing an explicit motivational context, have been also shown to elicit a prioritized processing reflected on modulations of several emotionrelated ERP components as indicated by the value-driven attention mechanism (B. A. Anderson, 2013) and the theoretical framework of motivated attention (Lang, Bradley, Cuthbert, et al., 1997). The effects of acquired motivational salience were mainly investigated using associative learning paradigms (e.g., Della Libera \& Chelazzi, 2009), which allow the investigation of the influences of acquired salience without interference with stimulusdriven salience. Associative learning is based on Thorndike's law of effect (Thorndike, 1927) and on classical or operant conditioning (Dickinson, 1981; Mackintosh, 1983; Pavlov, 1927; Skinner, 1953, for reviews). Skinner (1953) proposed that behavior, which is reinforced, is strengthened and will be repeated. Therefore, in line with the proposed value-driven attention mechanism (B. A. Anderson, 2013), also inherently neutral stimuli associated with motivational salience (positive-negative, reward-loss) via associative learning were shown to modulate selected emotion-related ERP components. Ventura-Bort et al. (2016) paired a set of neutral objects (e.g., tools or kitchen equipment) with emotional (positive, neutral, or negative) background pictures (IAPS; Lang, Bradley, \& Cuthbert, 1997). They could 
show that the perceptual (P1) and the elaborate processing (LPC) of neutral objects was impacted by previous associations with emotional backgrounds compared to neutral backgrounds. Schacht, Adler, Chen, Guo, and Sommer (2012) associated previously unknown Chinese words with a monetary outcome (gain, loss, or no outcome). During delayed testing, enhanced P1 and LPC amplitudes were detected for stimuli which were previously associated with monetary reward, whereas EPN modulations were absent. Recently, using a highly similar associative learning paradigm, Rossi et al. (2017) associated unknown letters with monetary outcomes (gain, loss, or no outcome), and found that the P3, suggested to underlie the LPC component (Luck, 2014), was modulated by letters previously associated with monetary gain. However, also negative associated motivational salience was shown to impact the visual processing of inherently neutral stimuli; especially the earliest ERP component, $\mathrm{C} 1$, which peaks around $60-90 \mathrm{~ms}$ after stimulus onset and is linked to the first response of the primary visual cortex (Di Russo et al., 2003), was modulated by associations of threat-related pictures (Stolarova, Keil, \& Moratti, 2006) and associated loss (Rossi et al., 2017). As mentioned above, also motivational context, mainly provided by reward- and loss-indicating cues preparing the organism to react rapidly to such incentives (Scheuthle, Carabias-Hütter, \& Kaiser, 2005), was shown to elicit a preferential processing. For instance, it has been found that an increase of the motivational salience of a given context can be generated by introducing reinforcements as incentives (Bayer et al., 2017; Meadows, Gable, Lohse, \& Miller, 2016). This finding was supported by subsequent studies showing that the presentation of a motivational cue, which indicates the possibility of monetary gain, elicits not only a cue-P3 directly after cue onset (Zheng et al., 2017), but also impacts stimulus processing over consecutive stages from EPN-related sensory encoding to LPC-related higher-order evaluations (Wei, Wang, \& Ji, 2016).

In particular human faces have been assumed to be inherently salient, as reflected for instance, in the face-superiority effect (e.g., Crouzet et al., 2010; Langton et al., 2008). Further, they seem to be susceptible for contextual influences (Morel, Beaucousin, Perrin, \& George, 2012; Wieser \& Brosch, 2012, for a review). In particular, neutral facial expressions were shown to be sensitive to context modulations, potentially due to the challenge to interpret them in social situations without additional information (Wieser \& Brosch, 2012). Several ERP studies investigated the impact of (emotional) context on neutral facial expressions. Aguado et al. (2012) associated neutral facial expressions with subsequent faces expressing emotions (happy, neutral, or angry). During delayed testing, neutral faces previously associated with angry faces elicited P1 modulations, whereas EPN modulations were restricted to inherently angry facial expressions presented during the learning phase. Wieser et al. (2014) associated neutral facial expressions with affective verbal descriptions (positive, neutral, or negative) and demonstrated enhanced EPN amplitudes for neutral faces associated with negative verbal descriptions. In a similar manner, Suess, Rabovsky, and Abdel Rahman (2014) reported larger EPN effects to faces associated with affectively negative compared to faces associated with neutral (fictive) biographical knowledge. It was shown that neutral expressions previously associated with negative verbal information modulated the emotion-related EPN component during delayed testing. These findings 
suggest that neutral facial expressions might also be susceptible for associations with motivational salience (in line with the value-driven attention mechanism), however, to date, no ERP evidence has been provided. In contrast, the neural processing of facial expressions of emotion was recently demonstrated to be modulated by associated motivational salience. Yao, Ding, Qi, and Yang (2014) reported that the prioritized processing of angry facial expressions can be extenuated by reward associations on the N2pc component, linked to spatial attention (Kiss, Van Velzen, \& Eimer, 2008). Interactions of emotional expressions and associated reward were further found on reaction times (Wei \& Kang, 2014), but only when the facial expression was task-relevant. In contrast, studies investigating emotional words found that emotion- and reward-related effects show different topographies and occur in different time windows (Kaltwasser, Ries, Sommer, Knight, \& Willems, 2013). These conflicting findings lead to the assumption that a systematic integration of stimuli associated with motivational and carrying inherent emotional salience is mandatory to gain further insights in neural processing similarities or differences.

However, not only ERPs were used to investigate impacts of facial expressions of emotion or motivational salience. In addition, pupil dilation, an indicator for physiological arousal (e.g., Bradley, Miccoli, Escrig, \& Lang, 2008), was demonstrated to increase in response to angry facial expressions (e.g., Kret, Roelofs, Stekelenburg, \& de Gelder, 2013) as well as to monetary reward (Massar, Lim, Sasmita, \& Chee, 2016) and loss (Pulcu \& Browning, 2017). Further, electromyography (EMG) was used to provide further insight into the processing of faces expressing positive (zygomaticus major) and negative (corrugator supercilia) emotions (e.g., Dimberg, 1982; Dimberg \& Petterson, 2000). In particular, zygomaticus major activation seems to be sensitive to associated motivational salience, as previously reward-associated neutral faces elicited an increased zygomaticus response when the same identity was presented with a happy expression compared to novel identities with happy expressions which were not previously associated with motivational salience (Sims, Van Reekum, Johnstone, \& Chakrabarti, 2012).

In summary, previous research suggested that neutral stimuli can acquire salience via associative learning (Rossi et al., 2017; Schacht et al., 2012). The question whether neutral facial expressions might gain motivational salience via associations with a monetary outcome remained, however, open. Previous research mainly focused on the impacts of reward on visual processing (e.g., Bourgeois, Chelazzi, \& Vuilleumier, 2016; Della Libera \& Chelazzi, 2009; Hickey, Chelazzi, \& Theeuwes, 2010; Vuilleumier, 2015, for a review), yet, ERP evidence with regard to whether the impacts of associated monetary reward and loss are comparable in human face processing is largely missing. This might be due to the fact that explicit learning paradigms typically result in an unbalanced outcome of associated reward and loss, as successful learning commonly implies an increase of monetary outcome (e.g., Schacht et al., 2012). Further, whether impacts of inherent emotional and associated motivational salience might be integrated in human face processing remains still unclear (e.g., Yao et al., 2014). Therefore, as face stimuli can be controlled adequately (e.g., for luminance) and neutral expressions seem to be susceptible to contextual modulations (e.g., Aguado et al., 2012; Wieser \& Brosch, 2012, for a review), associative learning provides 
an excellent approach to compare effects of inherent emotional salience (faces expressing happiness, anger or neutrality) and associated motivational salience (neutral faces associated with monetary gain, loss or no outcome) in an overall relevant stimulus domain. This dissociation will help to gain insights in the potential similarities and differences of their neural processing. Moreover, it will help in answering open questions with regard to whether (1) neutral faces, in comparison to facial expressions of emotion, can gain salience via associative learning; (2) impacts of associated reward and loss are symmetric and (3) inherent emotional and associated motivational salience might even be integrated.

\section{Aim and overview of present studies}

A wide variety of studies investigated either the impacts of inherent emotional salience or associated motivational salience on (emotion-related) ERP components. To date, no study intended a direct comparison between these two types of salience, although the assumption of the value-driven attention mechanism (B. A. Anderson, 2013) indicates that both emotionally and motivationally relevant stimuli elicit prioritized processing. To investigate the neural mechanisms of motivational salience associated to human faces and to compare them to typical emotion-related ERP components elicited by inherent facial expressions of emotion, three studies (Studies 1 to 3 in Chapters 2 to 4 ) were conducted. In Chapter 5, results of the studies will be discussed and integrated into a broader research context.

In Study 1, neutral facial expressions were explicitly associated with a monetary outcome (reward, loss, or no outcome) via an associative learning paradigm (adapted from Schacht et al., 2012). The participants had to successfully learn whether a neutral face was reward-, loss-, or zero outcome-associated, which was controlled by a required learning criterion. During delayed testing, previously associated neutral faces were presented together with facial expressions of emotion (happy, angry, and neutral) of either familiarized or novel identities to directly compare effects of associated motivational and inherent emotional salience on typical emotion-related ERP components (P1, N170, EPN, and LPC component).

In Study 2, a different task was used to investigate whether ERP modulations of associated motivational salience to neutral facial expressions might also occur via implicit associative learning and to directly compare those implicitly associated neutral faces to facial expressions of emotion. During the learning session, a motivational cue was presented at the beginning of every trial indicating the potential outcome of the subsequent trial. A facial prime was presented followed by a target face. A prime-face matching task with a subliminal prime was used to assure task performance on chance level and, thus, equalized reward and loss associations. During delayed testing, implicitly associated neutral faces from the learning session were presented together with novel identities with facial expressions of emotion to compare effects on typical emotion-related ERP components. The advantage of this experimental design is that it allows the examination of the symmetry of impacts of monetary reward and loss. Outcomes were equalized during the learning session due to a task performance on chancel level assuring an equalization of 
performance-dependent reward, loss, or zero outcome conditions. Impacts of motivational context elicited by the motivational cue at the beginning of the trials during learning were investigated both on ERPs and on pupil dilations. Pupil dilations were recorded to trace effects of arousal elicited by the motivational cue during learning and delayed testing, when the motivational cue is absent.

As associated motivational and inherent emotional salience were directly compared in Study 1 and 2, Study 3 aimed at the integration of both types of salience to gain further insights in their dissociation. To this aim, happy as well as neutral expressions were explicitly associated with monetary reward or no outcome during a learning session (comparable to the experimental design of Study 1). The learning phase finished when the participants reached the required learning criterion and was directly followed by a consolidation phase while the task remained the same. In addition to ERPs, the zygomaticus major activity was measured to investigate whether an integration of associated motivational and inherent emotional salience took place.

Whereas, the first part of this thesis (Chapter 1) addressed theoretical, methodological and empirical issues related to the dissociation of inherent emotional and associated motivational salience, the second part opens the experimental section, where three ERP studies (Chapter 2 to 4) will be discussed separately. The last part (Chapter 5) summarizes all important results in a general discussion. In addition, limitations and implications for future research will be discussed. The thesis will close with conclusions gained from this empirical work. 


\title{
Chapter 2
}

\section{Associated Motivational Salience Impacts Early Sensory Processing of Human Faces}

\begin{abstract}
Facial expressions of emotion have an undeniable processing advantage over neutral faces, discernible both at behavioral level and in emotion-related modulations of several eventrelated potentials (ERPs). Recently it was proposed that also inherently neutral stimuli might gain salience through associative learning mechanisms. The present study investigated whether acquired motivational salience leads to processing advantages similar to biologically determined origins of inherent emotional salience by applying an associative learning paradigm to human face processing. Participants $(N=24)$ were trained to categorize neutral faces to salience categories by receiving different monetary outcomes. ERPs were recorded in a subsequent test phase consisting of gender decisions on previously associated faces, as well as on familiarized and novel faces expressing happy, angry or no emotion. Previously reward-associated faces boosted the P1 component, indicating that acquired reward-associations modulate early sensory processing in extrastriate visual cortex. However, ERP modulations to emotional - primarily angry - expressions expanded to subsequent processing stages, as reflected in well-established emotion-related ERPs. The present study offers new evidence that motivational salience associated to inherently neutral stimuli can sharpen sensory encoding but does not obligatorily lead to preferential processing at later stages. ${ }^{1}$
\end{abstract}

Keywords: Motivational Salience, Emotional Expression, Associative Learning, Visual Cortex, Event-related Brain Potentials (ERPs).

\footnotetext{
${ }^{1}$ Hammerschmidt, W.; Sennhenn-Reulen, H., \& Schacht, A. (2017). Associated motivational salience impacts early sensory processing of human faces. NeuroImage, 156, 466-474. doi: $10.1016 /$ j.neuroimage.2017.04.032
} 


\title{
Chapter 3
}

\section{Implicit Reward Associations Impact Face Processing: Time-resolved Evidence from Event-related Brain Potentials and Pupil Dilations}

\begin{abstract}
The present study aimed at investigating whether associated motivational salience causes preferential processing of inherently neutral faces similar to emotional expressions by means of event-related brain potentials (ERPs) and changes of the pupil size. To this aim, neutral faces were implicitly associated with monetary outcome, while participants $(N=44)$ performed a subliminal face-matching task that ensured performance around chance level and thus an equal proportion of gain, loss, and zero outcomes. Motivational context strongly impacted processing of all - even task-irrelevant - stimuli prior to the target face, indicated by enhanced amplitudes of subsequent ERP components and increased pupil size. In a separate test session, previously associated faces as well as novel faces with emotional expressions were presented within the same task but without motivational context and performance feedback. Most importantly, previously gain-associated faces amplified the LPC, although the individually contingent face-outcome assignments were not made explicit during the learning session. Emotional expressions impacted the N170 and EPN components. Modulations of the pupil size were absent in both motivationally-associated and emotional conditions. Our findings demonstrate that neural representations of neutral stimuli can acquire increased salience via implicit learning, with an advantage for gain over loss associations. ${ }^{1}$
\end{abstract}

Keywords: Event-related brain potentials (ERPs), Implicit associative learning, Motivational context, Motivational salience, Pupil dilations.

\footnotetext{
${ }^{1}$ Hammerschmidt, W., Kagan, I., Kulke, L., \& Schacht, A. (2017). Implicit reward associations impact face processing: Time-resolved evidence from event-related brain potentials and pupil dilations. bioRxiv. doi: https://doi.org/10.1101/232538
} 


\subsection{Introduction}

To support adaptive behavior in complex environments, the human brain developed efficient selection mechanisms that bias perception in favor of salient information. In order to address the variety of different sources of salience, conventional attention theories focusing on goal- and salience-driven attention mechanisms (Connor et al., 2004; Corbetta \& Shulman, 2002) were extended by the assumption of a fundamental value-driven attention mechanism (B. A. Anderson, 2013; Failing \& Theeuwes, 2017, for a recent review). This mechanism is discernible not only in stimuli inherently carrying salience, but also in stimuli associated with motivational valence, all sharing similar attentional prioritization. In line with this account, not only physical stimulus features but also emotional and motivational factors have been demonstrated to determine increased salience of certain stimuli and directly impact attention and visual processing capacities (e.g., Zeelenberg, Wagenmakers, \& Rotteveel, 2006), resulting in a facilitated sensory encoding at initial processing stages (e.g., Della Libera \& Chelazzi, 2006). Stimuli of particularly high inherent salience are faces, for which involuntarily capture of attention and preferential processing has been documented, presumably due to their crucial role in human social interactions. This face-superiority effect has been reliably demonstrated on a behavioral level in object recognition/perception tasks (e.g., Langton et al., 2008), and moreover in studies employing visual search tasks or attentional blink paradigms including facial expressions of emotions (A. K. Anderson, 2005; Calvo \& Lundqvist, 2008; Eastwood, Smilek, \& Merikle, 2001; Vuilleumier, 2005). Particularly, facial expressions of emotions convey various types of relevant information in social interactions (for a review, see Frith, 2009) and are considered as evolutionary prepared stimuli (e.g., Vuilleumier \& Pourtois, 2007). Faces with and without emotional expressions are thus ideal stimuli in experiments investigating inherent versus associated salience effects as they allow for a direct comparison within an overall relevant stimulus domain. Due to their high temporal resolution, event-related brain potentials (ERPs) allow segregating different processing stages and therefore gaining insights to the mechanism underlying the face superiority effect as well as the processing advantage of facial expressions of emotions over time. By means of ERPs, several studies indicated that the processing of facial expressions of emotion elicit amplified neural responses compared to other visual stimuli such as pictures of affective scenes or written words of emotional content (Bayer \& Schacht, 2014; Schacht \& Sommer, 2009a). Attentional priority for facial expressions of emotion and their sustained preferential processing over neutral faces is reflected in several dissociable ERP components (e.g., Rellecke et al., 2012; Schupp et al., 2004). Especially two ERP components have been linked to subsequent stages of emotion processing in humans: the EPN and the LPC. The Early Posterior Negativity (EPN), a relative negativity over posterior electrode sites, typically starting around 150-200 ms after stimulus onset (e.g., Junghöfer, Bradley, Elbert, \& Lang, 2001; Rellecke et al., 2011), has been suggested to reflect enhanced sensory encoding of facial expressions of emotion. The EPN is typically followed by the Late Positive Complex (LPC) or Late Positive Potential (LPP, e.g., 
Cuthbert et al., 2000; Schupp et al., 2004) over centro-parietal electrodes, starting around $300 \mathrm{~ms}$ after stimulus onset (e.g., Rellecke et al., 2011). This long-lasting ERP response has been assumed to reflect higher-order elaborate and evaluative processes (for a review, see Olofsson et al., 2008; Rellecke et al., 2011; Schacht \& Sommer, 2009a). In addition, two earlier components were recently found to be modulated by emotional expressions. First, the P1 component, is peaking around $100 \mathrm{~ms}$ after stimulus onset, consisting of bilateral occipital positivities and reflecting the activation of extrastriate visual areas via selective attention (Di Russo et al., 2003). Enhanced P1 amplitudes have been reported for emotional facial expressions in comparison to neutral facial expressions (e.g., Batty \& Taylor, 2003; Rellecke et al., 2011), indicating that emotional salience impacts early perceptual encoding. Second, the N170 has been functionally linked to holistic face perception, consisting in a negativity over temporo-occipital electrodes (e.g., Bentin et al., 1996). As the evidence of N170 modulations by emotional expressions is inconsistent (for reviews, see Hinojosa et al., 2015; Rellecke et al., 2013), the question whether configural and emotional features of a face are processed independently (Bruce \& Young, 1986) could yet not finally be answered.

Facial expressions of emotions, as well as other stimuli of emotional content, carry an increased motivational salience, e.g., angry faces trigger the avoidance system, while happy faces might carry reward in social interactions. Previous studies have demonstrated that even neutral faces gain salience through associated emotional context information (Suess et al., 2014; Wieser et al., 2014). However, in particular motivational salience might arise from a variety of other sources, driven by first, an explicit motivational context or second, by acquired associations. Contexts might determine motivational dispositions - e.g., the readiness to act in given situations - as they can confront a person with appealing opportunities and daunting obstacles (Scheuthle et al., 2005) and thus directly influence behavior. An increase of the motivational salience of a given context can be generated by introducing reinforcements as incentives (Meadows et al., 2016). In a recent ERP study, Wei et al. (2016) showed that the expectation of monetary gain - indicated by motivationally relevant cues - impacted stimulus processing over consecutive stages from sensory encoding (EPN) to higher-order evaluation (P3/LPC). Interestingly, motivational incentives have been recently demonstrated to affect stimulus processing even before effects of spatial attention (Bayer et al., 2017). In addition, a "cue-P3" component directly elicited after cue onset with enhanced amplitudes for reward-indicating as compared to loss-indicating cues was reported (Zheng et al., 2017).

Driven by the compelling evidence for impacts of motivational contexts and inherent emotional valence, the question arises under which conditions salience can be acquired. The value-driven attention mechanism proposed by Anderson (B. A. Anderson, 2013) incorporated this question suggesting that processing advantages are not restricted to stimuli of emotional content (e.g., facial expressions of emotion), but also hold for stimuli that have been associated with reward, even if these are inherently non-salient or task-irrelevant. A fruitful approach to test this assumption is provided by associative learning paradigms that allow the investigation of the influences of acquired salience without interference 
with stimulus-driven salience. Aiming at a direct comparison between inherent and associated saliences, Hammerschmidt, Sennhenn-Reulen, and Schacht (2017) reported that explicit reward-associations to inherently neutral faces elicited increased P1 responses during delayed testing. The elicitation of typical emotion-related ERP components at longer latencies (EPN and LPC), was, however, restricted to facial expressions of emotion. In contrast, employing a highly similar learning paradigm as in the study by Hammerschmidt, Sennhenn-Reulen, and Schacht (2017), Rossi et al. (2017) detected an increase of the P3 to reward-associated unknown single letters from unfamiliar alphabets. Importantly, the processing advantage reported for stimuli associated with motivational salience is not restricted to rewards but has also been demonstrated for associations with aversive events (Hintze, Junghöfer, \& Bruchmann, 2014; Stolarova et al., 2006) or monetary loss (Rossi et al., 2017), mainly present on the perceptual level.

ERPs reflect processing differences on the neural level but cannot directly be linked to physiological arousal - one of the key components of emotions (Lang \& Bradley, 2010; Scherer, 2005, 2009). Physiological arousal is reflected amongst other indicators in changes of the pupil size, which have been related to norepinephrine release in the locus coeruleus (Berridge \& Waterhouse, 2003; Einhäuser, Stout, Koch, \& Carter, 2008; Gilzenrat, Nieuwenhuis, Jepma, \& Cohen, 2010; Laeng, Sirois, \& Gredebäck, 2012; Murphy, O'Connell, O'Sullivan, Robertson, \& Balsters, 2014). Therefore, pupil activity can be used as a measure of attentional, cognitive and emotional processing (Kang, Huffer, \& Wheatley, 2014; Smallwood et al., 2011) with increased pupil size in response to emotionally arousing pictures (Bradley et al., 2008) and auditory stimuli (Partala \& Surakka, 2003). In particular, inherently angry faces paired with an angry body induced larger pupil dilations than fearful and happy face-body pairs (Kret et al., 2013). Moreover, motivational modulations through outcome associations, in addition to stimuli of inherent emotional salience, can also increase pupil size, demonstrated for both reward (e.g., Massar et al., 2016) and loss incentives (Pulcu \& Browning, 2017). Interestingly, modulations of pupil dilation further depend on task difficulty, manipulated through mental effort (Mathôt, Siebold, Donk, \& Vitu, 2015; Peysakhovich, Causse, Scannella, \& Dehais, 2015), and decision uncertainty (Brunyé \& Gardony, 2017; Kahneman, 1973; Satterthwaite et al., 2007; Urai, Braun, \& Donner, 2017), with greater pupil dilations occurring with increasing task difficulty. The parallel measurement of ERPs, pupil dilations and behavioral data might help elucidate the multiple components involved in emotion processing (e.g., Grandjean, Sander, \& Scherer, 2008).

In line with Anderson's assumption (B. A. Anderson, 2013) of a value-driven attention mechanism, suggesting shared mechanisms of inherent bottom-up stimulus attention and context- or learning-based salience effects, previous research clearly indicated that both emotional and motivational aspects have a direct impact on visual stimulus processing. Nevertheless, the specific conditions, under which learning mechanisms or different contexts can modify a certain stimulus' salience, are not fully understood, presumably contributing to heterogeneous findings in the past. Despite the great progress in this area of research, there are a number of outstanding open questions that have not sufficiently 
been addressed: Firstly, effects of associated motivational salience occurred during several processing stages mainly in explicit associative learning paradigms (e.g., Hammerschmidt, Sennhenn-Reulen, \& Schacht, 2017; Hintze et al., 2014; Rossi et al., 2017; Stolarova et al., 2006). However, it seems reasonable that motivation or emotion-based salience might have been acquired implicitly, that is without explicit knowledge about the hedonic value of the certain stimulus. Hence, one of the yet unresolved questions is whether implicit and explicit associations of motivational salience have similar effects on stimulus processing. Implicit learning is generally linked to participants/learners' problems with an explicit recall (Berry \& Dienes, 1993), often characterized as a 'complex form of priming' (Cleeremans, Destrebecqz, \& Boyer, 1998). Further, it was argued that implicit representations possibly need more time and cognitive resources to be generated than information learned explicitly (Batterink \& Neville, 2011). Recently, it could be demonstrated that reward associations have a direct impact on spatial attention - even when presented implicitly (Bourgeois, Neveu, \& Vuilleumier, 2016). Secondly, it remained open whether the impacts of associated gain and loss might be symmetric under conditions of equalized outcomes, as successful learning usually implies an increase of gain in parallel to reduced losses (e.g., Hammerschmidt, Sennhenn-Reulen, \& Schacht, 2017; Rossi et al., 2017).

The main aim of the current study was to investigate potential effects of implicitly learned associations of motivational salience to neutral facial stimuli in direct comparison to effects elicited by inherent facial expressions of emotion. To this aim, we employed a prime-face matching task with subliminal prime presentation, implementing performance at chance level and thus an equalization of performance-dependent gain, loss, or zero-outcome conditions. During the learning session, colored cues were presented at the beginning of each trial, indicating the motivational condition which was kept constant for each of the inherently neutral target faces. During the test phase, the same task was employed, however without any performance-depended monetary incentives and feedback. In addition to the previously learned neutral faces, facial expressions of emotion of novel identities were presented, allowing for a comparison of effects driven by associated motivational and inherent emotional salience. We collected ERP and pupil size data during the learning and test sessions with the main aim to test the impact of motivational contexts on subsequent stimulus processing (cf., Wei et al., 2016) and to allow the investigation of the temporal characteristics and autonomous physiological correlates of association-related effects on the following day. We expected that the cue-indicated reward or loss would boost sensory processing of task-relevant face stimuli in the visual cortex (Bayer et al., 2017), resulting in enhanced P1 amplitudes after target face onset. Aiming at expanding the findings by Zheng et al. (2017) that showed augmented P3 amplitudes elicited by reward-indicating visual cues, we further tested potential modulations of cue-evoked ERP potentials by different motivational contexts. As the incentive values of the cue stimuli were made explicit to our participants, these simple symbolic stimuli might carry increased salience as stimuli of emotional/motivational content and thus trigger increased amplitudes of EPN and LPC components. Pupil dilations should be increased in condition of high motivational salience (Massar et al., 2016; Pulcu \& Browning, 2017). For faces associated with monetary gains 
on the previous day, we expected increased amplitudes of early ERP components (e.g., P1; Hammerschmidt, Sennhenn-Reulen, \& Schacht, 2017). Loss-associations might trigger similar effects as gain-associations as both incentive conditions were equalized - in terms of frequency of occurrence and amount of monetary outcome - during the learning session. Faces with happy and particularly with angry expressions should elicit larger EPN and LPC amplitudes than neutral expressions (e.g., Rellecke et al., 2011; Schacht \& Sommer, 2009a; Schupp et al., 2004). For pupil dilations, we expect an increase for angry compared to happy and neutral expressions (Kret et al., 2013). Pupil dilations to neutral faces associated with motivational salience the day before might show no increase due to the absence of arousing motivational context.

\subsection{Materials and Methods}

\section{Participants}

Data was collected from fifty-five participants. Seven participants were excluded due to EEG artifacts in either the learning or test phase, and four due to strategies that successfully countered visual masking during the face-matching task (the performance exclusion criterion was defined as an individual performance-dependent bonus exceeding average bonus $\pm 2 S D s$ across participants in the learning session). The remaining forty-four participants ( 21 female) were ranging in age between 18 and 32 years (mean age $=24.0$ years, $S D$ $=3.5$ ), with normal or corrected-to-normal vision and without neurological or psychiatric disorders according to self-report. Forty-two participants were right-handed (according to Oldfield, 1971). Participants received 8 euro per hour or course credit; in addition, the individual monetary bonus achieved during the learning phase was disbursed.

\section{Stimuli}

Facial stimuli were selected from the Karolinska Directed Emotional Faces (KDEF) database (Lundqvist, Flykt, \& Öhman, 1998). Twelve colored pictures of faces (6 female, 6 male) with neutral facial expressions were used as target faces. The same pictures served as primes in matching trials; additional pictures of neutral faces (6 female, 6 male) were used as nonmatching primes. An ellipsoid mask surrounded all facial stimuli within an area of $130 \times 200$ pixels $\left(4.59 \times 7.06 \mathrm{~cm}, 4.6 \times 7.1^{\circ}\right)$ in order to eliminate hair, ears and clothing and leave only the face area visible. For the learning phase, diamond-shaped cues of 120 x 120 pixels $(3.18 \times 3.18 \mathrm{~cm})$ were generated that indicated the outcome category (reward, loss, zero outcome) of the given trial in three different equi-luminant colors (blue, pink, and brown). Grey circles were used as feedback stimuli ( $248 \times 248$ pixels, $5 \times 5$ $\mathrm{cm}$ ) indicating the amount of monetary outcome won or lost in the preceding trial in the corresponding cue color. For the test phase, twelve novel identities with facial expressions of emotion (happy, neutral, angry, $N=36$ colored pictures) were presented in addition to the neutral faces which were presented during the learning phase the day before both as target faces and matching primes. Another twelve new identities (6 female, 6 male) showing facial expressions of emotion (happy, neutral, angry, $N=36$ colored pictures) 
were used as prime stimuli in non-matching trials. For each face stimulus (in total $N=$ 96), a scrambled version was generated and used as mask for the preceding primes. All facial stimuli were matched offline for luminance (according to Adobe Photoshop CS6 ${ }^{T M}$ ), $F(23,72)=0.873, p=0.631$. All stimuli were presented in the center of the screen on a light gray background.

\section{Procedure}

The study was conducted in accordance with the Declaration of Helsinki and approved by the local ethics committee of the Institute of Psychology at the University of Göttingen. Participants were informed about the procedure of the study and gave written informed consent prior to both phases of the experiment. The study consisted of a learning and a test phase, which were completed on two subsequent days. Participants were seated in a dimly lit, sound-attenuated room, in front of a computer screen (refresh rate $100 \mathrm{~Hz}$ ) at a distance of $57 \mathrm{~cm}$. Participants placed their chin and forehead on a head rest in order to avoid movements and ensure correct recording of pupil sizes. After pupil diameter calibration, participants received detailed instructions about the experimental task.

In the learning phase, twelve inherently neutral faces were implicitly associated with monetary gain, loss, or no outcome via an associative learning paradigm. At the beginning of each trial, a diamond-shaped cue indicated the monetary outcome context condition (gain, loss, or neutral: no gain/loss). The assignment of the cue's color was fixed for each participant but counterbalanced across participants. The meaning of the cues and the feedback scheme was explained prior to the experiment. Participants were asked to decide whether the identity of the presented target face was matching the preceding prime face - irrespective of the presented cue. In the gain condition, the correct classification of the face-matching task was awarded with +50 cents (incorrect classifications $=0$ cents). A correct classification in the loss condition prevented the participants from the loss of money (0 cents), whereas an incorrect classification led to a loss of 50 cents. For the neutral condition, feedback was either +0 cents (correct classification) or -0 cents (incorrect classification). Responses were given by a button press; correct/incorrect-buttons as well as prime-target assignments were counterbalanced, but consistent within one participant. In the face-matching task, prime and target faces differed in $50 \%$ of the trials in identity, but were always matched with respect to gender. In case the participant missed to answer a trial within $5000 \mathrm{~ms}$, 70 cents were removed from the bonus. Stimuli were presented blockwise with a total of 20 blocks. Each block consisted of the 12 target faces with neutral expressions presented twice in randomized order, paired with a matching (50\%) or a non-matching (50\%) prime, resulting in 480 trials in total. Importantly, the cue-target face associations remained stable during the learning phase for each participant, but were counterbalanced in order to exclude any potential effects of physical stimulus features on the ERP components of interest. At the beginning of each trial (see Figure 3.1), a fixation cross was presented in the center of the screen for $1000 \mathrm{~ms}$, followed by the diamond-shaped cue, which was visible for $500 \mathrm{~ms}$. Subsequently, a fixation cross was shown for $200 \mathrm{~ms}$ followed by the prime face for $10 \mathrm{~ms}$. The mask appeared for $200 \mathrm{~ms}$ followed by a fixation 


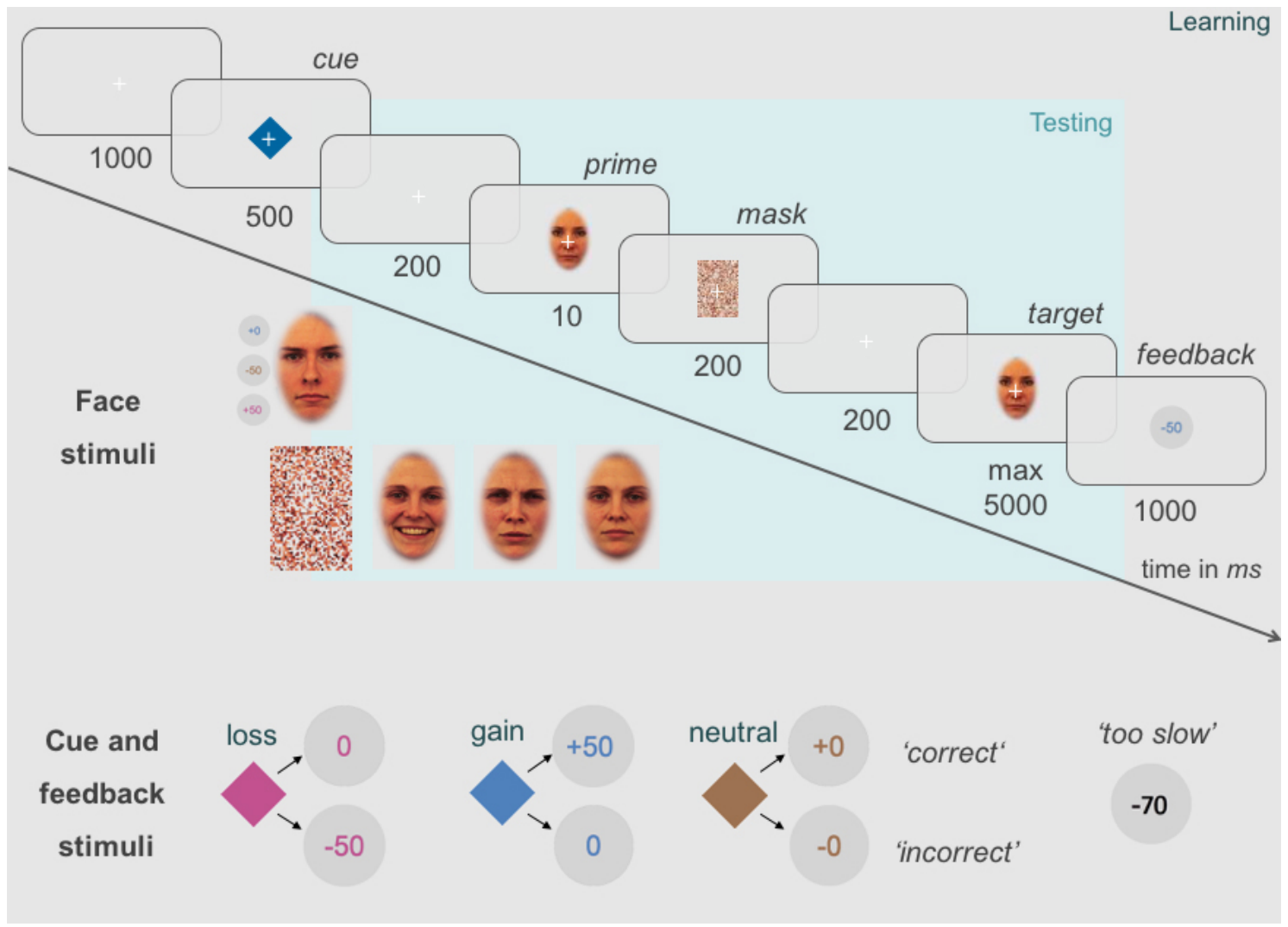

Figure 3.1: Trial scheme of the learning and test session with detailed time sequence.

cross for $200 \mathrm{~ms}$. The target face was shown up to $5000 \mathrm{~ms}$, disappearing with button press. The feedback was displayed for $1000 \mathrm{~ms}$. Blocks were separated by a self-determined break, in which the current amount of the individual bonus was displayed. Participants started with a base pay of 10 euro and achieved an individual monetary bonus according to their performance ranging between -11 and 18 euro (mean $=1.11$ euro, $S D=5.98$ euro); participants finishing the learning session with a negative balance received the full base payment of 10 euro. In order to check whether the associations of the presented cue and the target face remained implicit, a manipulation check was implemented at the end of the learning phase. The twelve target face identities were presented simultaneously, randomly arranged on the computer screen. The participants were asked to explicitly assign them to one of the three outcome contexts (gain/neutral/loss). This task was repeated about 30 minutes later.

The test phase took place on the following day, to allow for memory consolidation. The face-matching task remained constant, however, no cue or corresponding feedback was provided, and participants could not win or lose any money. The test phase consisted of two different types of facial stimuli presented blockwise. Half of the blocks consisted of the twelve neutral target faces, which were implicitly associated with monetary outcome context the day before. The other half of the blocks consisted of twelve novel identities with emotional facial expressions (4 for, happy, neutral, and angry, respectively) serving as target face and primes in matching trials, and twelve additional novel identities with emotional expressions (4 for, happy, neutral, and angry, respectively) serving as primes in 
the non-matching trials. Target and prime faces always matched with respect to gender and emotional expressions. As in the learning phase, each target face was presented twice with a matching and a non-matching prime in randomized order $(N=48$ blocks $)$. The trial scheme was identical to the learning session, except that cues and feedback stimuli were excluded (see Figure 3.1). Each block was repeated ten times in randomized order, resulting in 20 blocks and 960 trials in total per face condition. The blocks were separated by breaks of self-determined length. Again, a manipulation check was conducted at the end of the test phase: all 24 target face identities with neutral expressions (from both blocks with previously learned and inherent facial expressions) were presented on the computer screen in random order. The participants were asked for each face whether it was presented during the learning phase the day before or during the test phase for the first time.

\section{Acquisition and pre-processing of ERP and pupil data}

The EEG was recorded from 64 electrodes, placed in an electrode cap (Easy-Cap, Biosemi, Amsterdam, Netherlands) according to the international 10-20 system (Pivik et al., 1993). The common mode sense (CMS) electrode and the driven right leg (DRL) passive electrodes were used as reference and ground electrodes (http://www.biosemi.com/faq/cms\&drl .htm). Six external electrodes were used: Two on the left and right mastoids respectively, and four external electrodes were placed on the outer canthi and below the eyes to record eye movements and blinks. Signals were recorded at a sampling rate of $512 \mathrm{~Hz}$ and a bandwidth of $102.4 \mathrm{~Hz}$ (http://www.biosemi.com/faq/adjust_filter.htm), offline filtered with a Low Cutoff $(0.03183099 \mathrm{~Hz}$, Time constant 5 s, 12 dB/oct), a High Cutoff $(40 \mathrm{~Hz}, 48 \mathrm{~dB} /$ oct $)$ and a Notch Filter $(50 \mathrm{~Hz})$. Data was processed using BrainVision Analyzer (Brain Products GmbH, Munich, Germany). Data was down-sampled to 500 $\mathrm{Hz}$, average-referenced and corrected for ocular artifacts (blinks) using Surrogate Multiple Source Eye Correction with default parameters (MSEC; Ille, Berg, \& Scherg, 2002) as implemented in BESA (Brain Electric Source Analysis, MEGIS Software GmbH, Gräfelfing, Germany). Application of Surrogate MSEC is detailed in Scherg (2003). The continuous EEG signal of the learning phase was segmented into epochs of $2310 \mathrm{~ms}$, starting $200 \mathrm{~ms}$ before cue onset and referred to a $200 \mathrm{~ms}$ pre-cue baseline. The continuous EEG signal of the test phase was segmented into epochs of $1610 \mathrm{~ms}$, starting $200 \mathrm{~ms}$ before prime onset and referred to a $200 \mathrm{~ms}$ pre-prime baseline. Based on previous research (Hammerschmidt, Sennhenn-Reulen, \& Schacht, 2017), time windows and regions of interest (ROIs) electrodes for ERP components were chosen as follows for the learning session (related to cue onset): P1 cue: 75-125 ms; EPN cue: 200-300 ms; LPC cue: 350-500 ms; P1 fixation cross1: 585-635 ms; P1 prime/mask: 760-810 ms; P2 prime/mask: 885-935 ms; P1 fixation cross2: 985-1035ms; P1 target: 1185-1235 ms; N170 target: 1240-1290 ms; EPN target: 1310-1460 ms; LPC target: 1460-1810 ms. For the test session (related to target face onset): P1: 75-125 ms, N170: 130-180 ms, EPN: 200-350 ms, P3: 200-350 ms, LPC: 350-700 ms. ERPs were quantified as most positive peak using peak detection (P1 at $\mathrm{O} 1$ and $\mathrm{O} 2$, reference electrode: O2; N170 at P9 and P10, reference electrode: P10; P2, O1 and O2, reference electrode: O2) or mean amplitudes (EPN at P9, P10, Iz, Oz, O1, O2, PO7, and 
PO8; LPC at Pz, P1, P2, CPz, and POz).

Pupil diameter was recorded binocularly using a desktop-mounted eyetracker (EyeLink 1000, SR Research) at a $500 \mathrm{~Hz}$ sampling rate. Prior to the experiment, pupil diameter was calibrated with an artificial pupil placed on the lid of the left eye of the participants to set the baseline for the measurement of the pupil dilation size. Offline, analyses of pupil diameter were performed using Matlab. Trigger codes of pupil and EEG data were synchronized. Data from two subjects were excluded due to technical failure of the eye tracker in the learning or test phase, respectively. For each participant and the learning and test sessions separately, artifacts were identified as samples in which the difference in pupil size to the subsequent sample was higher than $0.1 \mathrm{~mm}$ or the difference in pupil size from the median across the session was higher than $1 \mathrm{~mm}$. Artifacts were interpolated. Eleven subjects had to be excluded after artifact correction due to excessive artifacts that could not be interpolated in either the learning or the test session. The remaining pupil size data was segmented into epochs from $200 \mathrm{~ms}$ prior to cue (learning session)/prime (test session) onset to $7000 \mathrm{~ms}$ after. For each subject and condition, pupil size time courses were averaged across both eyes and correct and incorrect responses, and corrected to a baseline $200 \mathrm{~ms}$ before cue (learning session)/prime (test session) onset. Mean pupil size between 1500 and $4000 \mathrm{~ms}$ after cue/prime onset (based on the response latency after cue onset measured by Bayer et al., 2017) was computed for each subject and condition. One additional subject was excluded because the measured pupil size exceeded the average across subjects by more than 10 SDs.

\section{Data analyses}

All parameters - reaction times (RTs), accuracy (in percent), ERP peaks or mean amplitudes, and pupil diameter - were analyzed with repeated-measures (rm)ANOVAs, separately for the learning session and test session. Outliers were identified as reaction times (RTs) below $200 \mathrm{~ms}$ or exceeding $+2 S D s$ from the mean per condition and were excluded from behavioral data analysis. RmANOVAs on data from the learning session included the factor Motivation (gain, neutral, and loss). Data from the test phase were analyzed in separate rmANOVAs, including the factor Motivation (gain, neutral, and loss) for learned faces or the factor Emotion (happy, neutral, and angry) for novel faces with emotional expressions. Accuracy deviations from chance level, across the sample and on the individual subject level, were analyzed using the exact test for equality of several binomial proportions to a specified standard (Krishnamoorthy, Thomson, \& Cai, 2004; Unakafov, 2017). All post-hoc pair-wise comparisons were Bonferroni-corrected. 
Table 3.1: Mean reaction times in ms, accuracy in task and manipulation check in \%, during/after face-matching task in the learning session (SEMs in parentheses), contrasted for factor levels of Motivation.

\begin{tabular}{|c|c|c|c|c|}
\hline \multicolumn{5}{|c|}{ Learning Session } \\
\hline & \multicolumn{2}{|c|}{ Face-Matching Task } & \multicolumn{2}{|c|}{ Manipulation Check } \\
\hline & RTs & Accuracy & 1st Check & 2nd Check \\
\hline Gain & $1019(49)$ & $51(0.7)$ & $57(3.3)$ & $55(3.9)$ \\
\hline Neutral & $960(44)$ & $51(0.6)$ & $48(4.6)$ & $48(3.9)$ \\
\hline Loss & 1079 (51) & $51(0.7)$ & $45(3.4)$ & $47(3.7)$ \\
\hline
\end{tabular}

\subsection{Results}

\section{Effects of Motivational Context in the Learning Phase}

\section{Behavioral Data}

Descriptive values for behavioral performance measures of the learning session are provided in Table 3.1. Accuracy on the face-matching task during the learning phase was at 50\% chance level (not different from the expected random binomial distribution with 0.5 probability, $p>0.05$, Bonferroni-corrected), and was not impacted by the factor Motivation, $F(2,86)=0.149, p=0.850, \eta_{p}^{2}=0.003$. Mean reactions times (RTs) of the learning phase significantly differed as a function of the factor Motivation, $F(2,86)=24.929, p<0.001$, $\eta_{p}^{2}=0.367$, with increasing RTs from neutral to gain- and loss-context, and loss to gain context trials, all $F s(1,43)>11.206$, all $p s<0.006$, all $\eta_{p}^{2}>0.207$. Correct assignments of the target faces to motivation conditions - obtained directly after the learning phase (1st check) and after 30 minutes delay (2nd check) - were above $33 \%$ chance level for gainand neural-associated faces ( $p<0.05$, Bonferroni-corrected, the exact test for equality of several binomial proportions to a specified standard), but did not reach significance for loss-associated faces, without any performance improvement after 30 minutes delay, $F<$ 1.

\section{ERP Data}

ERPs elicited by motivational cues. EPN mean amplitudes between 200 and $300 \mathrm{~ms}$ after cue onset differed as a function of Motivation, $F(2,86)=7.960, p=0.001, \eta_{p}^{2}=0.156$, for gain- compared to neutral-, $F(1,43)=10.295, p=0.009, \eta_{p}^{2}=0.193$, and loss- compared to neutral-related trials, $F(1,43)=14.837, p<0.001, \eta_{p}^{2}=0.257$. LPC mean amplitudes between 350 and $500 \mathrm{~ms}$ after cue onset were also modulated by Motivation, $F(2,86)=$ $37.755, p<0.001, \eta_{p}^{2}=0.468$, with enhanced amplitudes for gain- compared to neutral, $F(1,43)=52.145, p<0.001, \eta_{p}^{2}=0.548$, for loss- compared to neutral-, $F(1,43)=$ 26.100, $p<0.001, \eta_{p}^{2}=0.378$, and for gain- compared to loss-related trials, $F(1,43)=$ 22.067, $p<0.001, \eta_{p}^{2}=0.339$. The P1 elicited by motivational cues was not impacted by the factor Motivation (see Figure 3.2). As can be seen in Figure 3.2, the impacts of motivational incentives were long-lasting. Therefore, ERPs between cue and target face presentation were analyzed to investigate potential impacts of motivational context. 


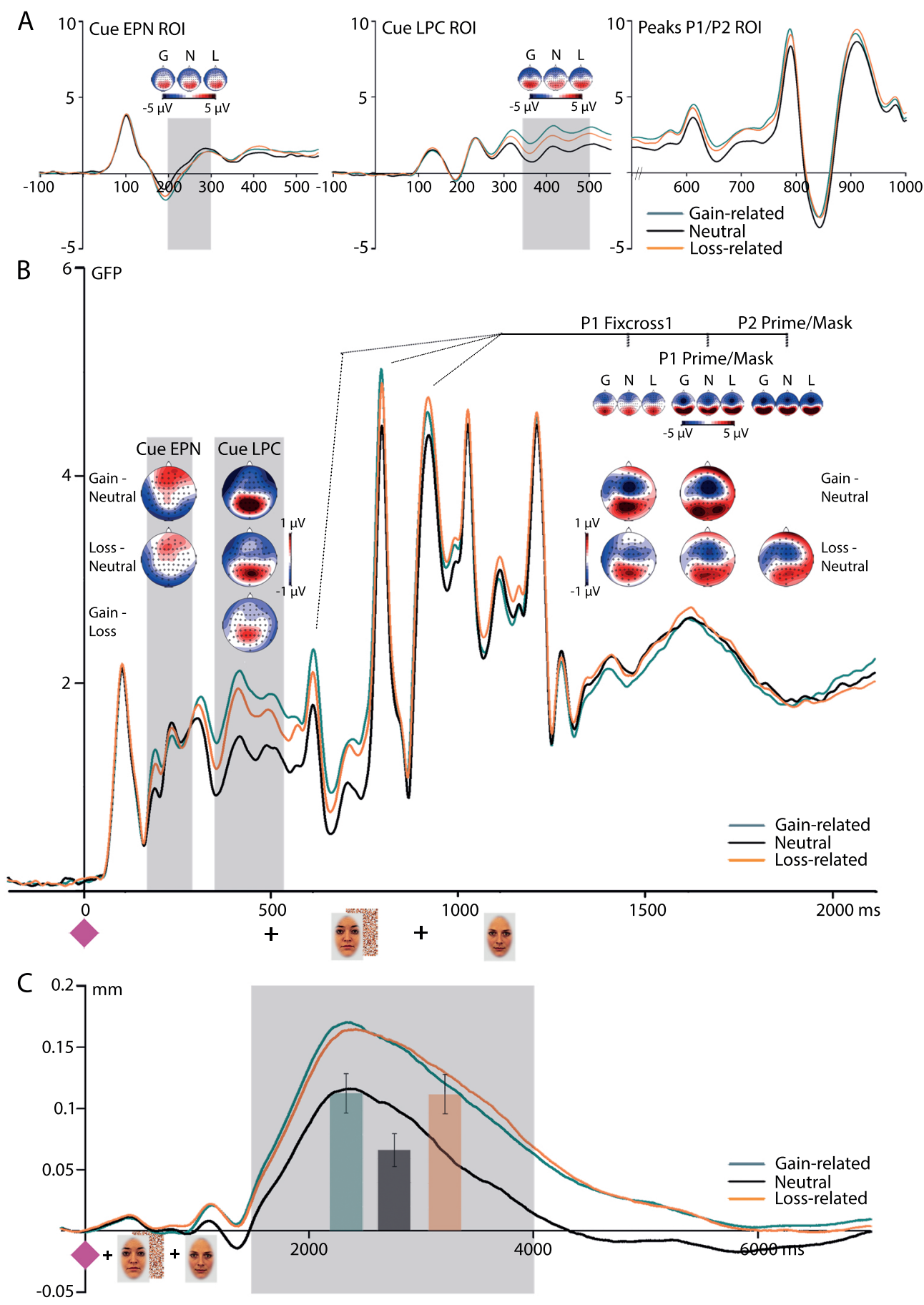

Figure 3.2: ERP effects of the learning phase for Cue-EPN and Cue-LPC for associated faces and the following peaks. A: Regions of interest (ROIs) for the corresponding analyses. B: GFP wave form of a complete trial for reward-, neutral- and loss-related faces including ERP topography of raw distributions (small topographies) and differences between indicated motivation categories. Highlighted areas display the time windows of Cue-ERP analyses, P1/P2 peaks of the after-cue/pre-target face interval were analyzed with peak detection. C: Pupil dilation responses to gain-, neutral-, and loss-related contexts, the highlighted area displays the time window of pupil dilation analysis with means and SEMs embedded as bar chart. 
The P1 component following the first fixation cross after cue presentation was modulated by the Factor Motivation, $F(2,86)=8.752, p=0.001, \eta_{p}^{2}=0.169$, with enlarged peak amplitudes for reward- compared to neutral-, $F(1,43)=16.513, p<0.001, \eta_{p}^{2}=0.277$, and loss- compared to neutral-related trials, $F(1,43)=7.115, p=0.033, \eta_{p}^{2}=0.142$. Motivation further influenced the $\mathrm{P} 1$ component following prime/mask, $F(2,86)=13.959$, $p<0.001, \eta_{p}^{2}=0.245$, with larger positivities for reward- compared to neutral-, $F(1,43)$ $=25.947, p<0.001, \eta_{p}^{2}=0.376$, and loss- compared to neutral-related trials, $F(1,43)=$ $10.699, p=0.006, \eta_{p}^{2}=0.199$. The visual $\mathrm{P} 2$ following prime/mask was also modulated by the Factor Motivation, $F(2,86)=5.934, p=0.005, \eta_{p}^{2}=0.121$, with enhanced peak amplitudes for loss- compared to neutral-related trials, $F(1,43)=10.981, p=0.006, \eta_{p}^{2}$ $=0.203$. The fixation cross response following the prime $/$ mask was not modulated by the factor Motivation anymore (see Figure 3.2, panels A and B).

ERPs to Target Faces. According to rmANOVAs, modulations of peak amplitudes for P1 and N170 components and mean amplitudes for EPN and LPC components by implicitly associated motivational salience were absent.

\section{Pupil dilations}

For pupil dilation data of the learning phase, an rmANOVAs showed a significant withinsubjects effect of Motivation, $F(2,58)=32.871, p<0.001, \eta_{p}^{2}=0.531$, with increased pupil diameters for gain- compared to neutral-, $F(1,29)=43.413, p<0.001, \eta_{p}^{2}=0.6$, and loss- compared to neutral-related trials, $F(1,29)=33.466, p<0.001, \eta_{p}^{2}=0.536$ (see Figure 3.2, panel C).

\section{Effects of Associated Motivational and Inherent Emotional Salience in the Test Phase}

\section{Behavioral Data}

Descriptive values for behavioral performance measures of the test session are provided in Table 3.2. In contrast to the learning session, the accuracy on the face-matching task in the test phase across the sample of 44 subjects was slightly above the $50 \%$ chance level $(\mathrm{Ms}$ $=51-53 \%, p<0.05$, Bonferroni-corrected). In particular, five subjects showed a significant accuracy above (4 subjects, accuracy 58-65\%) or below chance level (one subject, 40\%) across all three motivational conditions, for the previously associated faces $(p<0.05)$. Similarly, six subjects (four same as for the motivational conditions) showed above chance accuracy for novel faces across all three emotional conditions (58-70\%). Accuracy was not impacted by the factors Motivation/Emotion, and did not differ between conditions (learned faces /novel faces), Fs $<1.4$. During the test phase, RTs were not modulated by the Factor Motivation/Emotion, Fs $<1$. After the test phase, all 24 target faces from both learning and testing phase were presented to the participants (2 subjects did not complete the retrieval). They had to assign those to either the learned target faces from the day before or to the novel target faces with emotional expressions of the test phase (average performance: $M=84.0 \%, S E M=2.5 \%$ ) to control for familiarization with the target 
Table 3.2: Mean reaction times in ms, accuracy in task and manipulation check in \%, during/after face-matching task in the test session (SEMs in parentheses), contrasted for all factor levels of Motivation/Emotion.

\begin{tabular}{llll}
\hline & \multicolumn{3}{l}{ Test Session } \\
\hline & \multicolumn{4}{l}{ Face Matching Task } & Manipulation Check \\
\cline { 2 - 4 } RTs & Accuracy & Old/New \\
\cline { 2 - 4 } Reward & $986(57)$ & $51(1.0)$ & $87(3.0)$ \\
Neutral & $985(57)$ & $51(0.8)$ & $80(3.7)$ \\
Loss & $978(56)$ & $52(0.8)$ & $83(3.7)$ \\
\cline { 2 - 4 } Happy & $1011(58)$ & $53(0.9)$ & $86(3.2)$ \\
Neutral & $1006(55)$ & $51(0.9)$ & $78(4.0)$ \\
Angry & $1014(58)$ & $51(1.0)$ & $89(3.4)$ \\
\hline
\end{tabular}

faces of the learning phase. The factor Motivation did not impact accuracy of learned target faces. For novel target faces with emotional expressions, a main effect of the factor Emotion was detected, $F(2,82)=4.173, p=0.020, \eta_{p}^{2}=0.092$, with higher accuracy rates for angry compared to neutral expressions, $F(1,41)=7.280, p=0.030, \eta_{p}^{2}=0.151$.

\section{ERP Data}

ERP Effects of Associated Motivational Salience. RmANOVAs on ERPs revealed a significant main effect of the factor Motivation on LPC mean amplitudes for inherently neutral faces associated with motivational salience, $F(2,86)=10.632, p<0.001, \eta_{p}^{2}=0.198$, with increased amplitudes for gain-associated compared to neutral faces, $F(1,43)=18.792, p$ $<0.001, \eta_{p}^{2}=0.304$, and compared to loss-associated faces, $F(1,43)=8.880, p=0.015$, $\eta_{p}^{2}=0.171$ (see Figure 3.3). P1, N170, and EPN amplitudes to associated faces were not influenced by the Factor Motivation, when tested in the a-priori defined time windows and ROIs.

Further ERP Effects of Associated Motivational Salience prior to the LPC component. The time window 200-350 ms after target face onset, which revealed no EPN modulation for associated motivational salience, was visually re-inspected (see Figure 3.3) as amplitude distributions and corresponding topographies bore a high resemblance to the LPC effect (350-700 ms) of associated motivational salience outlined above. Therefore, the time window was reanalyzed with the centro-parietal LPC ROI revealing effects of associated motivational salience, $F(2,86)=5.124, p=0.008, \eta_{p}^{2}=0.106$, with enhanced amplitudes for gain- compared to neutral-associated faces, $F(1,43)=8.346, p=0.018, \eta_{p}^{2}=0.163$. ERP Effects to Facial Expressions of Emotion in Novel Identities. N170 peak amplitudes to the target faces were significantly impacted by the factor Emotion, $F(2,86)=7.901, p$ $=0.001, \eta_{p}^{2}=0.155$, with enhanced negativities for angry compared to neutral, $F(1,43)=$ 13.695, $p=0.003, \eta_{p}^{2}=0.242$, and happy expressions, $F(1,43)=8.941, p=0.015, \eta_{p}^{2}=$ 0.172. EPN mean amplitudes of novel emotional expressions were significantly modulated by the Factor Emotion, $F(2,86)=21.217, p<0.001, \eta_{p}^{2}=0.330$, with enhanced amplitudes for happy compared to neutral, $F(1,43)=34.587, p<0.001, \eta_{p}^{2}=0.446$, and for angry compared to neutral facial expressions, $F(1,43)=39.982, p<0.001, \eta_{p}^{2}=0.482$. P1 


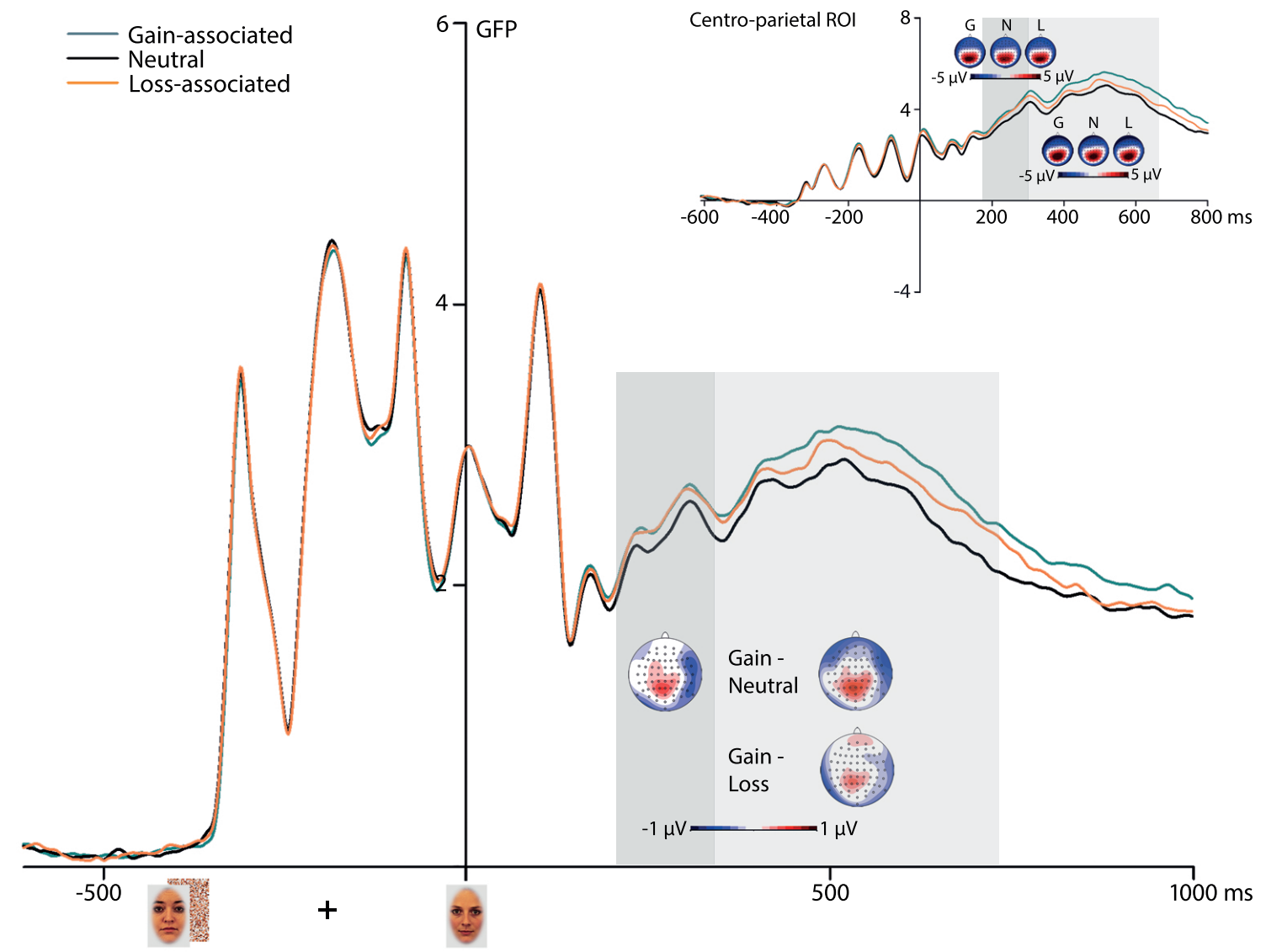

Figure 3.3: GFP wave form of a complete trial of the test session for gain-, neutraland loss-associated faces including centro-parietal/LPC ROI and ERP topography of raw distributions (upper graph) and differences between indicated motivation categories. Highlighted areas display the time windows of analyses.

peak and LPC mean amplitudes for novel faces with emotional expressions were unaffected by the Factor Emotion (see Figure 3.4).

\section{Pupil dilations}

An rmANOVA showed no significant within-subjects effect of associated motivational salience on pupil size, $F(2,58)=0.049, p=0.950, \eta_{p}^{2}=0.002$. Pupil size in response to novel facial stimuli with emotional expressions did not significantly differ, according to an rmANOVA, $F(2,58)=0.705, p=0.498, \eta_{p}^{2}=0.024$ (see Figure 3.5).

\section{Topography comparisons}

As there is no previous evidence for emotion/motivation-related ERP modulations following a motivational cue, it is an exploratory question whether a P3 modulation or an EPN modulation could be expected prior to LPC modulations driven by the valence of the cue. To decide whether the ERP difference modulations between 200-300 ms after cue onset resemble an EPN distribution, topography comparisons were measured. To this end, the mean amplitude of all 64 electrodes was divided by global field power (GFP; Skrandies, 


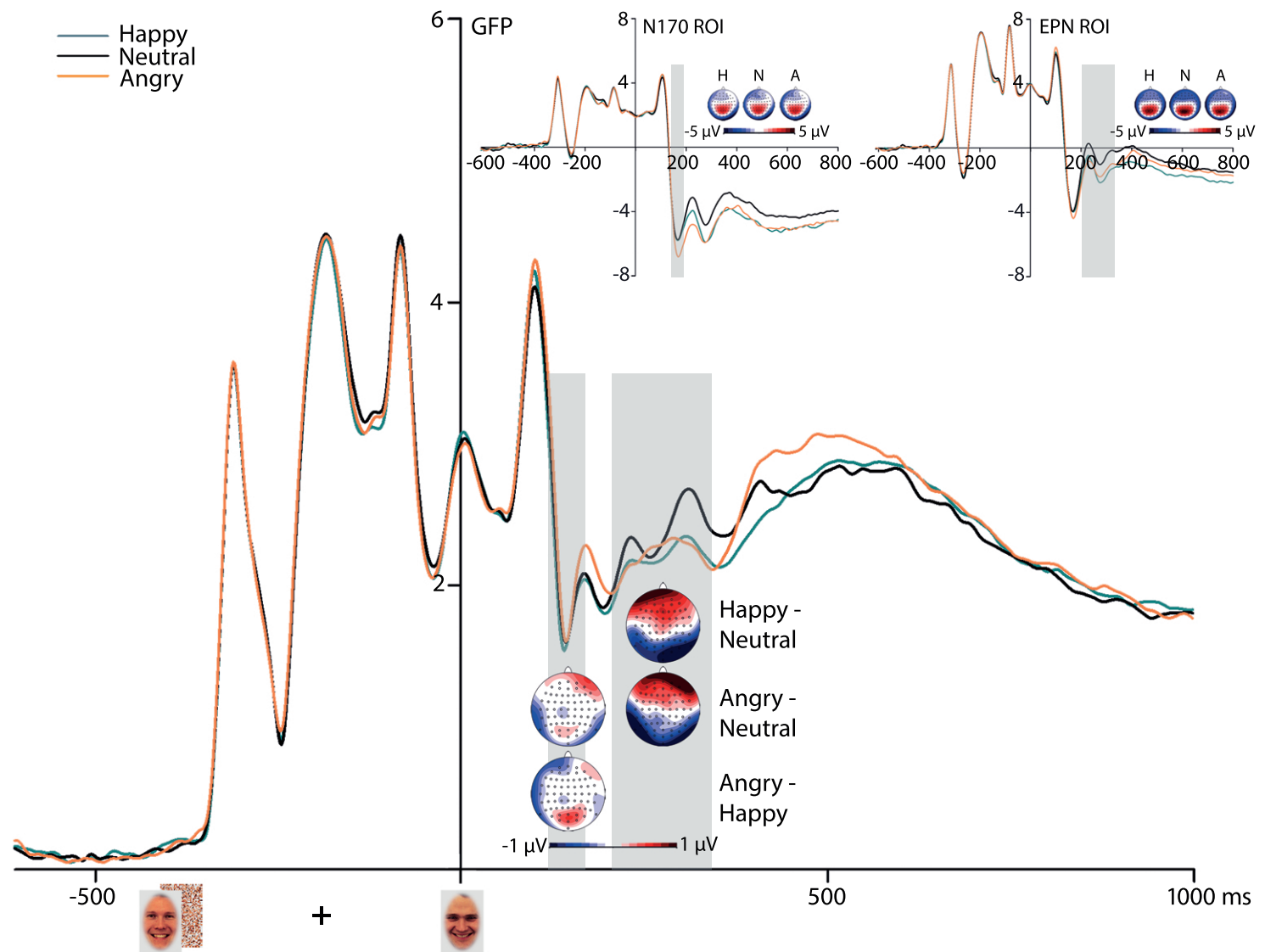

Figure 3.4: GFP wave form of a complete trial of the test session for happy-, neutral- and angry faces including N170/EPN ROIs and ERP topography of raw distributions (upper graphs) and differences between indicated emotion categories. Highlighted areas display the time windows of analyses.

1990) per condition respectively to extinguish amplitude differences. Difference of the particular conditions were measured and compared with the topography of an established ERP component via rmANOVAs with the factor Electrode (64) and the factor Topography (2). To compare the topography of the ERP modulation 200-300 after cue onset, the difference topography of gain minus neutral cues was compared with the difference topography of happy minus neutral expressions of the test phase. The topography $\mathrm{x}$ electrode interaction revealed no significant difference between these two topographies, $F<1$. Similarly, the difference topography of loss minus neutral cues was compared to the difference topography of angry minus neutral expressions of the test phase. The topography $\mathrm{x}$ electrode interaction again failed significance between these two topographies, $F<1.325$.

\subsection{Discussion}

The main aim of the present study was to investigate whether implicitly learned associations of motivational salience result in a prioritized processing similar to what has been previously shown for explicit associations or inherent emotional salience (e.g., Hammer- 

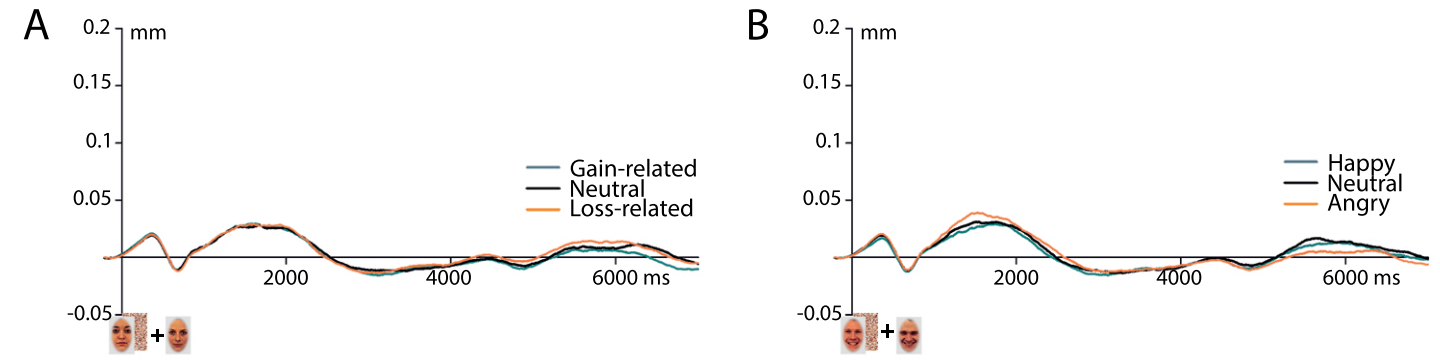

Figure 3.5: Pupil dilations during the test phase for A: previously associated and B: inherent emotional expressions.

schmidt, Sennhenn-Reulen, \& Schacht, 2017). Further, effects of motivational incentives on subsequent stimulus processing were examined during learning, while gain and loss were held equal in terms of their frequency and amount of monetary outcome. To address these aims, we implemented a multi-measure approach, considering ERPs as indicator of neural processing, pupil dilations as a correlate of arousal, and behavioral parameters as control variables. During a learning session, a sequential face-matching task using inherently neutral faces as subliminal and masked primes and supraliminal targets was employed, while motivational context was indicated by preceding cues and feedback about monetary outcome at the end of each trial. Importantly, target face assignments to motivational context were kept constant for each participant (but were counterbalanced between participants). On the following day, the previously associated faces were presented together with novel faces with expressions of emotion (happy, angry, and neutral faces) allowing for a direct comparison of potential effects driven by associated versus inherent salience during face processing.

\section{Implicitly acquired reward associations improve stimulus processing}

Our main finding is a long-lasting ERP effect of gain implicitly associated to inherently neutral faces that became evident from 200 to $700 \mathrm{~ms}$ after target face onset. Across the whole time window this ERP modulation consisted of increased centro-parietal positivities, presumably resembling P3 and LPC components - linked to higher-order stimulus evaluations - that were particularly boosted for gain-associated faces. Such modulations of late processing stages (P3/LPC) by monetary reward have been previously demonstrated in studies employing associative learning based on explicit valence categorization (Rossi et al., 2017; Schacht et al., 2012). These previous findings have been interpreted to indicate that previously rewarded stimuli receive increased cognitive resources, resulting in a prioritized processing (Nieuwenhuis, Aston-Jones, \& Cohen, 2005), even for implicit reward associations (Bourgeois, Neveu, \& Vuilleumier, 2016). In particular, the P3/LPC modulations on inherently neutral, but previously associated faces deserve special attention for two reasons: First, we did not find modulations of ERPs by motivational incentives after target face onset during the learning session. Second, the condition-to-face assignments were not made explicit for the participants during the learning session; indicating that the 
effect was driven by the implicit associations of the motivational contexts to certain faces. One potential explanation of these findings relates to the time required for consolidation that has been proposed in particular for arousing stimuli (Sharot, Delgado, \& Phelps, 2004). Therefore, overnight consolidation might play a crucial role particularly during the implicit association of motivational salience as similar P3 effects modulated by monetary reward were observed during an explicit learning paradigm without delay between learning and testing (Rossi et al., 2017). In contrast to previous associative learning studies, in particular to Hammerschmidt, Sennhenn-Reulen, and Schacht (2017) who detected P1 modulations driven by monetary reward associations, no ERP modulations at short latencies were found in the present study. Two reasons for this finding are conceivable: First, as early ERP effects of acquired salience were detected in studies employing explicit associative learning, implicitly learned associations might lead to less apparent impacts on perceptual encoding of the certain stimuli. Second, the task demands in the present study were exceptionally high and might have suppressed early ERP modulations (e.g., Pessoa, 2015a). In order to check whether the present study design actually allows for typical emotion-related ERP modulations, novel identities with facial expressions of emotion were presented in the same task during the test phase. Modulations of two emotion-related ERP components occurred: The face-sensitive N170 component was modulated by angry facial expressions compared to both neutral and happy expressions, supporting the assumption that the N170 is primarily (if at all) influenced by negative expressions (for reviews, see Hinojosa et al., 2015; Rellecke et al., 2013). It was further suggested that the N170 might be overlapped by the directly following EPN component which leads to comparable modulations by emotional expressions (Rellecke et al., 2011, 2012; Schacht \& Sommer, 2009a). For the EPN component, typical modulations were found for happy and angry compared to neutral facial expressions (e.g., Hammerschmidt, Sennhenn-Reulen, \& Schacht, 2017), as the EPN is known to reflect the automatic encoding of the emotional content of a given stimulus independent of task demands (Rellecke et al., 2011). In addition to N170 and EPN, previous studies reported even earlier (P1) or later LPC modulations (e.g., Hammerschmidt, Sennhenn-Reulen, \& Schacht, 2017; Rellecke et al., 2012; Schupp et al., 2004), but in the present study those modulations were absent, potentially due to the task-irrelevance of the expressed emotion. Therefore, the present study design indeed allows for typical emotion-related ERP modulations; however, P1 modulations, known to be task-dependent (Pratt, Willoughby, \& Swick, 2011; Rellecke et al., 2012), might therefore be suppressed by the high cognitive load of the task used in the present study.

\section{Motivational contexts boost subsequent processing of even task-irrelevant stim- uli}

Recent studies provided robust evidence for impacts of motivational context on target stimulus processing (e.g., Krebs \& Woldorff, 2017), interestingly taking place even before effects of spatial attention occur (Bayer et al., 2017). What has yet been largely neglected is the question whether the motivational salience of cue stimuli might lead to preferential processing similar to stimuli of varying emotional content, such as affective scenes or emo- 
tional expressions (cf., B. A. Anderson, 2013). Using cue stimuli of identical shape that only differed in color (counterbalanced), allowed us to investigate potential ERP modulations through the cues' meaning, by keeping visual features constant across conditions. Interestingly, we found increased ERP effects to gain- and loss-indicating cues that resembled typical ERP modulations driven by stimuli of emotional content across different domains, i.e. EPN and LPC effects (e.g., Bayer \& Schacht, 2014; Schacht \& Sommer, 2009a). This impression was verified by topography comparisons between these ERP responses to the cues during the learning session and to EPN effects elicited by emotional expressions during the test session in the present study. Importantly, the first visually evoked ERP component after cue onset - the P1 - did not differ as a function of the cues' motivational salience. As cue stimuli in the present study were perceptually identical besides variation in three equi-luminant colors, the lack of P1 effects indicate that previously reported P1 effects modulated by emotional valence (e.g., Pourtois et al., 2004; Rellecke et al., 2012) reflect rapid core-feature analysis under the precondition that these features are clearly discriminable (Fedota, McDonald, Roberts, \& Parasuraman, 2012). Impacts of motivational incentives were, importantly, not restricted to the processing of cues but extended to the subsequent processing of even task-irrelevant stimuli within trials of increased motivational salience during the learning session. These impacts, however, declined when the target face was presented. As studies using associative learning paradigms typically report stabilized associated effects on target processing, future research is needed to determine the emergence of those associated effects.

\section{Effects on pupil dilations}

In the learning session, pupil dilations were enlarged for both gain- and loss-related contexts compared to neutral contexts. These findings indicate increased arousal or attention triggered by motivational incentives (Massar et al., 2016; Pulcu \& Browning, 2017). In the test session, although LPC modulations driven by reward associations were detected on the neural level during, pupil size did not differ as a function of associated motivational salience, indicating that physiological arousal only increases when motivational incentives are directly available. Furthermore, pupil size was also not impacted by facial expressions carrying inherent emotional salience (although these elicited EPN modulations on the neural level), contradicting previous findings (Kret et al., 2013) and thus indicating that impacts of emotional expressions might be suppressed by the cognitive load of the task and the consequential task-irrelevance of the expressed emotion.

\section{Impacts of monetary gain and loss under conditions of equalized outcomes}

In contrast to recent studies, which typically linked incentives explicitly to successful learning, the present study design ensured equalized outcomes of monetary gain and loss, but nevertheless demonstrated a prioritized neural processing of gain over loss. The influential prospect theory in economic decision making (Kahneman \& Tversky, 1979; Tversky \& Kahneman, 1992) already suggested an asymmetric function of gains and losses - with a typically higher impact of losses than gains during risky choices. This asymmetry is potentially 
based on the activation of different brain areas (Trepel, Fox, \& Poldrack, 2005), especially during reinforcement learning tasks (Kim, Yoon, Kim, \& Hamann, 2015; Wächter, Lungu, Liu, Willingham, \& Ashe, 2009). In contrast, visual selective attention studies revealed an advantage of gains over losses in the prioritized processing (for a revirew, see Barbaro, Peelen, \& Hickey, 2017; Chelazzi, Perlato, Santandrea, \& Della Libera, 2013). Recently, a first explanation for these seemingly conflicting assumptions was proposed based on findings that gain-associated targets were processed faster than loss-associated targets (Chapman, Gallivan, Wong, Wispinski, \& Enns, 2015). The authors concluded that the inhibition necessary for loss aversion takes more time than the facilitated processing elicited by reward associations.

\section{Conclusion}

The present findings demonstrate that motivational contexts impacted pupil dilation and led to an ongoing influence on the neural processing of subsequent visual stimuli (fixation cross, prime/mask) during the learning session, however, not persisting to the target faces. During the test session, implicitly associated motivational salience impacted the processing of neutral faces, reflected in an enhanced centro-parietal ERP modulation for previously gain-associated target faces. In contrast, target faces expressing emotions (happy, angry) modulated the typical emotion-related EPN component, whereas P1 and LPC modulations were suppressed presumably by high demanding task requirements. In summary, this study provides new evidence that neural representations of neutral stimuli can acquire increased salience via implicit learning, with an advantage for gain over loss associations.

\section{Funding}

This work was funded by the German Research Foundation (grant \#SCHA1848/1-1 to AS) and by the Leibniz ScienceCampus Primate Cognition (grants to AS and IK).

\section{Acknowledgments}

The authors thank Anna-Maria Grimm and Rebecca Jacob for their contributions to the development of the study design and data collection, Florian Niefind and Kay Reimers for their technical support during experimental setup, and Benthe Kornrumpf and Anton Unakafov for providing codes for data analyses. 


\title{
Chapter 4
}

\section{Money or Smiles: Independent ERP Effects of Associated Monetary Reward and Happy Faces}

\begin{abstract}
In comparison to neutral faces, facial expressions of emotion are known to elicit attentional prioritization, mainly demonstrated by means of event-related potentials (ERPs). Recent evidence indicated that such a preferential processing can also be gained by neutral faces when associated with increased motivational salience via reward. It remains, however, an open question, whether impacts of inherent emotional salience and associated motivational salience might be integrated. In the present study, participants $(N=42)$ learned to categorize happy and neutral faces as reward- and zero-outcome-related via an associative learning paradigm. After successful learning, a consolidation phase followed to strengthen the learned associations. ERPs were recorded throughout the experiment. In the learning phase, happy faces boosted the face-sensitive N170 and the emotion-related EPN component, compared to neutral faces, whereas effects of associated motivational salience were absent. In the subsequent consolidation phase, happy faces again elicited enhanced N170 and EPN amplitudes, while reward-associated faces - irrespective of their expressions amplified the LPC, a component linked to higher-order evaluations. Interactions between expressions and associated outcome conditions were absent in all ERP components of interest. The present study offers new evidence that acquired salience impacts stimulus processing but independent of the effects driven by happy facial expressions. ${ }^{1}$
\end{abstract}

Keywords: Motivational Salience, Emotional Expression, Associative Learning, Eventrelated Brain Potentials (ERPs).

\footnotetext{
${ }^{1}$ Hammerschmidt, W., Kulke, L., Bröring, C., \& Schacht, A. (2018). Money or smiles: Independent ERP effects of associated monetary reward and happy faces. bioRxiv. doi: https://doi.org/10.1101/325829
} 


\subsection{Introduction}

Because of limited cognitive resources, the human brain has evolved efficient selection mechanisms that bias perception in favour of salient, i.e. behaviourally relevant or physically distinct, information. Stimuli of increased salience have been demonstrated to directly capture attention and impact visual processing capacities (e.g., Vuilleumier \& Pourtois, 2007; Zeelenberg et al., 2006), resulting in facilitated sensory encoding even at initial processing stages (e.g., Della Libera \& Chelazzi, 2006). Faces, and in particular facial expressions of emotion, were demonstrated to be especially salient, as they not only provide important information about others in social interactions, but also have an intrinsic relevance to assure survival and well-being. Therefore, it has been assumed that humans have evolved a biological preparedness to rapidly detect emotional expressions (e.g., Öhman \& Mineka, 2001). For facial expressions of emotion, a preferential processing has been unveiled both at the behavioral and neural level, mainly for angry facial expressions (e.g., Recio, Schacht, \& Sommer, 2014; Recio, Sommer, \& Schacht, 2011; Rellecke et al., 2011, 2012; Schupp et al., 2004). However, for happy facial expressions a recognition advantage has been demonstrated (Kirita \& Endo, 1995), manifested in an increased and faster recognition accuracy compared to other facial expressions potentially based one the exclusive role of happiness as a positive expression (Calvo \& Lundqvist, 2008; Leppänen \& Hietanen, 2004). In addition, as humans are highly social beings, facial expressions of emotion are not only emotionally relevant, but also motivationally relevant, as, for instance, a happy face might carry a rewarding value similar to other reinforcers (Rolls, 2013). Traditional theories of attention focused on bottom-up (i.e., stimulus-driven) and top-down (i.e., goal-directed) attention mechanisms (e.g., Corbetta \& Shulman, 2002) to explain how relevant stimuli are preferentially processed. However, such accounts have recently been challenged by studies demonstrated a preferential processing of previously reward-associated stimuli, which occurs even when the stimuli themselves do not carry increased salience, when they are task-irrelevant, or when the reward is suspended over time (B. A. Anderson, 2013). To fill this gap, Anderson proposed a general value-driven attention mechanism to explain the attentional prioritization of not only stimuli of inherent emotional salience but also of stimuli that acquired their salience through learning processes. Supporting evidence for this assumption comes from studies indicating overlapping neural activity in the ventromedial prefrontal cortex (vmPFC) and ventral striatum, elicited by both emotional facial expressions and monetary reward (Lin, Adolphs, \& Rangel, 2012). Furthermore, motivational relevance has been widely equated with emotional stimulus valence or seen as a precursor of emotional significance in some scientific approaches (Lang \& Bradley, 2010; Pessoa, 2015b). This led to the obvious investigation of potential modifications of inherently emotional stimuli through learned associations of motivational salience.

An excellent tool to gain insights into the neuro-cognitive mechanisms underlying the prioritized processing of emotional stimuli are event-related brain potentials (ERPs) since they allow dissociating between different processing stages. In the domain of facial expressions of emotion, a large number of studies revealed rather robust modulations of dissociable ERP components over time: The Early Posterior Negativity (EPN), a typical 
emotion-related ERP component reflecting an enhanced sensory encoding of stimuli carrying inherent salience, starts around 150-200 ms after stimulus onset (e.g., Recio et al., 2011; Rellecke et al., 2012; Schupp et al., 2004) and has been demonstrated to be elicited by happy expressions (Bublatzky, Gerdes, White, Riemer, \& Alpers, 2014; Calvo \& Beltrán, 2013; Hammerschmidt, Sennhenn-Reulen, \& Schacht, 2017; W. Sato, Kochiyama, Yoshikawa, \& Matsumura, 2001). Another ERP component of interest in emotion processing is the Late Positivity Complex (LPC, or LPP; e.g., Schupp et al., 2004). The LPC is linked to higher-order stages of stimulus evaluation, developing around $300 \mathrm{~ms}$ and lasting for several hundred milliseconds (e.g., Rellecke et al., 2011). The ERP component is typically modulated by angry expressions, presumably due to their increased evolutionary relevance (Schupp et al., 2004), however, also happy expressions might modulate the LPC component (Bublatzky et al., 2014; Recio et al., 2011; Rellecke et al., 2012). Moreover, the P1 component, peaking around $100 \mathrm{~ms}$ at occipital electrodes, presumably reflects rapid activation of the extrastriate visual cortex (Di Russo et al., 2003) and is mainly impacted by negative expressions (e.g., Hammerschmidt, Sennhenn-Reulen, \& Schacht, 2017; Pourtois et al., 2004; Rellecke et al., 2012). The N170 component, typically following the P1 in face processing, is an occipito-temporal negativity linked to holistic face perception (Bentin et al., 1996). Several studies reported N170 modulations by emotional expressions, including happy expressions (Bublatzky et al., 2014; Marinkovic \& Halgren, 1998), but the conjuncture of N170 modulations by emotional expressions is still on debate (for reviews, see Hinojosa et al., 2015; Rellecke et al., 2013). In addition to these robust ERP effects elicited by facial expressions, also neutral faces associated with motivational salience were reported to impact dissociable ERP components over time. A recent study by Hammerschmidt, Sennhenn-Reulen, and Schacht (2017) directly compared the neural correlates of processing facial expressions of emotion and neutral faces associated with motivational salience by means of ERPs. Interestingly, reward-associated neutral faces elicited enhanced amplitudes of the P1 component, similar to P1 amplification by angry facial expressions. Whereas the prioritization of associated motivational salience was restricted to initial processing stages (P1), beneficial processing of facial expressions of emotions spread over to subsequent stages of more elaborative stimulus processing (EPN, LPC). In other studies, however, neutral faces implicitly associated with monetary reward have been shown to elicit enhanced LPC amplitudes (Hammerschmidt, Kagan, Kulke, \& Schacht, 2017), replicating previous findings that the LPC component seems to be sensitive to reward associations (Rossi et al., 2017; Schacht et al., 2012). According to the value-driven attention mechanism (B. A. Anderson, 2013), the processing of inherent emotional and associated motivational salience should share certain similarities. However, previous evidence could not finally answer this question, as effects of associated motivational salience were not demonstrated to lead to a sustained attentional prioritization over processing stages, as suggested for facial expressions of emotion. Therefore, both types of salience need to be directly integrated to investigate to what extent they share similar or even common processing characteristics, a question to which previous evidence is inconclusive. Interactions of associated reward and emotional expression were reported on reaction times (Wei 
\& Kang, 2014), but only when the facial expression was task-relevant. Further, Yao et al. (2014) demonstrated that the preferential processing of angry expressions can be extenuated through reward associations. However, the authors only investigated effects on the N2pc component, linked to spatial attention (Kiss et al., 2008), and disregarded the investigation of emotion-related ERP components. The present study aims at clarifying whether the preferential processing of inherently happy facial expressions might be impacted by motivational salience (reward) acquired via explicit associative learning, using a paradigm similar to Hammerschmidt, Sennhenn-Reulen, and Schacht (2017). Since in that study, effects of associated salience were restricted to the reward condition, here only happy and neutral faces were orthogonally associated with gain or zero-outcome respectively. Directly after reaching a pre-defined learning criterion, a consolidation phase was added to strengthen the learned associations. ERPs were recorded to compare the effects of the factors expressions, outcome and their potential interaction over different stages of face processing. Replicating previous findings, we hypothesized reward associations to be learned faster than zero-outcome-associations (Hammerschmidt, Sennhenn-Reulen, \& Schacht, 2017; Kulke, Bayer, Grimm, \& Schacht, 2017; Rossi et al., 2017), as well as faster reaction times for reward compared to zero-outcome-associations (Hammerschmidt, Sennhenn-Reulen, \& Schacht, 2017). In line with the literature, happy faces were expected to trigger the typical emotion-related EPN component (e.g., Hammerschmidt, SennhennReulen, \& Schacht, 2017; Rellecke et al., 2012; W. Sato et al., 2001) both in the learning and consolidation phase. A reward-modulation on the P1 component was expected for neutral faces associated with monetary gain (Hammerschmidt, Sennhenn-Reulen, \& Schacht, 2017). In addition, if associated motivational and inherent emotional salience are interacting on sensory processing stages, happy faces associated with reward were expected to elicit even stronger P1 modulations. The potential interaction of emotional expression and associated outcome was investigated on all measurements, behavioral data and ERPs. Finally, the changes in participants' mood by overall increasing monetary gain during the experiment were assessed.

\subsection{Materials and Method}

\section{Participants}

Data was collected from 48 participants. Four participants were excluded, as they did not reach the required learning criterion within 10 to 30 blocks, two due to artifacts. The remaining forty-two participants (20 female) had an age range between 19 and 30 years (mean age $=23.9$ years, $S D=2.7$ ), normal or corrected-to-normal vision and no neurological or psychiatric disorders according to self-report. All participants were right-handed (according to Oldfield, 1971) and were reimbursed by their individual bonus, ranging between 38.10 and 59.70 euro $(M=49.82$ euro, $S D=5.47$ euro). 


\section{Stimuli}

Sixteen colored facial stimuli ( 8 female, 8 male) were selected from the Karolinska Directed Emotional Faces (KDEF) database (Lundqvist et al., 1998) showing happy and neutral expressions respectively. A grey ellipsoid mask, ensuring a uniform figure/ground contrast, surrounded the stimuli within an area of $130 \times 200$ pixels $(4.59 \times 7.06 \mathrm{~cm})$ and let only the internal face area visible. Facial stimuli were matched for luminance across conditions (according to Adobe Photoshop CS6 ${ }^{T M}$ ), $F(1,30)=2.907, p=0.099$, and were presented at a central position on the screen on a light gray background, corresponding to a visual angle of $4.6^{\circ} \times 7.1^{\circ}$. Feedback symbols were presented in grey circles in the center of the screen (248 x 248 pixels, $5 \times 5 \mathrm{~cm}$ ) and were constructed perceptually identical (800 pixels respectively): a green plus (correct reward condition), a dark grey equality sign (correct zero-outcome condition) or a red cross (error). Feedback colors were equi-luminant.

\section{Procedure}

The study was conducted in accordance with the Declaration of Helsinki and approved by the local Ethics committee of the Institute of Psychology at the University of Goettingen. Participants were informed about the procedure and gave written informed consent prior to the experiment. Participants were seated in a dimly lit, sound-attenuated room at a viewing distance of $57 \mathrm{~cm}$ to the computer screen. During the experiment, 8 inherently happy and neutral expressions were associated with monetary gain, or no outcome via an associative learning paradigm (adapted from Hammerschmidt, Sennhenn-Reulen, \& Schacht, 2017). The participants' task was to learn the correct outcome-expression assignment for each of the faces presented. As no test trials were provided, the first block had to be answered by chance. The feedback scheme was explained prior to the experiment: Faces that had to be categorized as reward-related were associated with +20 cents (in case of correct classification) or -10 cents (incorrect classification). For faces that had to be categorized as zero-outcome-related, feedback was either 0 cents (correct) or -10 cents (incorrect). If the participants missed to answer a trial within $5000 \mathrm{~ms}, 50$ cents were removed from their bonus. Responses were given by button press; response-to-button assignment was balanced across participants, as well as face-to-expression/outcome assignment, but remained stable for each participant. Stimuli were presented block-wise, each block consisted of all sixteen facial stimuli in fully randomized order. The experiment consisted of 40 blocks (640 trials in total), separated by a self-determined break and information about the current amount of the individual bonus. A learning criterion was defined (48 of the last 50 trials correct) to assure successful learning. If the learning criterion was not reached within 10 to 30 blocks, data was excluded from analysis $(N=4)$. The remaining trials, until reaching 40 blocks, were presented to allow for consolidation. A black fixation point ( $5 \times 5$ pixels) was presented for $2000 \mathrm{~ms}$ in each trial, followed by the face for maximum $5000 \mathrm{~ms}$, disappearing with button press. Afterwards, a blank screen for $1500 \mathrm{~ms}$ and the feedback symbol for $1000 \mathrm{~ms}$ were presented; the inter-trial interval was $1000 \mathrm{~ms}$. While ERPs were recorded, a chin rest was used. The German Multidimensional Mood Questionnaire (MDBF; Steyer, Schwenkmezger, Notz, \& Eid, 1997) was completed before and after the task. 


\section{EEG Recording, Pre-processing and Analyses}

The electroencephalogram (EEG) was recorded from 64 electrodes, placed in an electrode cap (Easy-Cap, Biosemi, Amsterdam, Netherlands) according to the extended 10-20 system (Pivik et al., 1993). The common mode sense (CMS) electrode and the driven right leg (DRL) passive electrode were used as reference and ground electrodes (cf., http:// www. biosemi.com/faq/cms\&drl.htm). Six external electrodes were used, inferior and laterally to the eyes to record blinks, and on the left and right mastoids. Signals were recorded at a sampling rate of $2048 \mathrm{~Hz}$ (downsampled to $500 \mathrm{~Hz}$ for ERP analysis) and a bandwidth of $104 \mathrm{~Hz}$ (http://www.biosemi.com/faq/adjust_filter.htm), offline filtered with a Low Cutoff $(0.03183099 \mathrm{~Hz}$, Time constant $5 \mathrm{~s}, 12 \mathrm{~dB} /$ oct), a High Cutoff ( $40 \mathrm{~Hz}$, $48 \mathrm{~dB} /$ oct $)$, and a Notch Filter $(50 \mathrm{~Hz})$. Data was processed with BrainVision Analyzer (Brain Products GmbH, Munich, Germany), average-referenced and corrected for blinks using Surrogate Multiple Source Eye Correction with default parameters (MSEC; Ille et al., 2002) as implemented in BESA (Brain Electric Source Analysis, MEGIS Software GmbH, Gräfelfing, Germany). Application of Surrogate MSEC is detailed in Scherg (2003). The continuous EEG signal was segmented into epochs of $1200 \mathrm{~ms}$ (both for analysis of face and feedback stimuli), starting $200 \mathrm{~ms}$ before stimulus onset referring to a $200 \mathrm{~ms}$ pre-stimulus baseline. Electrodes with a noisy or no signal were interpolated by spherical splines in BrainVision Analyzer (Order of splines: 4; maximal degree of Legendre Polynomials: 10; Lambda: 1E-05). Epochs containing artifacts (criteria: voltage steps $>50 \mu \mathrm{V}, 200 \mu \mathrm{V} / 200$ ms intervals difference of values, amplitudes exceeding $-150 \mu \mathrm{V} / 150 \mu \mathrm{V}$, activity $<0.5 \mu \mathrm{V}$ ) were eliminated. Segments were averaged per Subject, Phase (2 - learning, consolidation), Expression (2 - happy, neutral) and Outcome (2 - reward, zero outcome). Based on a previous study (Hammerschmidt, Sennhenn-Reulen, \& Schacht, 2017), time windows and regions of interest (ROIs) electrodes for target face-related ERP components were chosen as follows: i) P1: 75-125 ms, O1 and O2; ii) N170: 130-180 ms, P9 and P10; iii) EPN: 200350 ms, P9, P10, Iz, Oz, O1, O2, PO7 and PO8; iv) LPC: 350-700 ms, Pz, P1, P2, CPz and POz. P1 components were quantified as the most positive peak (with $\mathrm{O} 2$ as reference electrode), N170 component as the most negative peak (with P10 as reference electrode); EPN and LPC,were quantified as mean amplitudes. For statistical analysis, repeated-measures (rm)ANOVAs were computed, including the factors, Expression (2 - happy, neutral) and Outcome (2 - reward, zero outcome) for the learning and consolidation phase respectively.

\section{Analyses of behavioral data}

To investigate the differences in learning curves between conditions, posterior distributions for the probability (the coefficient $\mathrm{p}$ of a Bernoulli distribution) to attribute the outcome category correctly were modeled. The number of trials until the learning criterion was met differed between participants. To account for these differences in trial number, the proportion of time (until the learning criterion was met) was considered (see Figure 4.1). Significant differences between these learning curves were defined based on a criterion of non-overlapping 99\% simultaneous credible bands (for more details, see Hammerschmidt, Sennhenn-Reulen, \& Schacht, 2017). For reaction times and accuracy data, repeated- 
measures (rm)ANOVAs were computed, including the factors, Expression (2, happy, neutral) and Outcome (2, reward, zero outcome).

\subsection{Results}

\section{Learning Phase}

\section{Behavioral Data}

Participants $(N=42)$ learned the outcome associations adequately within 10 to 26 blocks ( $M=17.2$ blocks, $S D=4.8$ blocks). In the happy face condition, reward-associated faces were learned faster, differing from zero-outcome-related faces from the beginning until $53.9 \%$ of the learning criterion was met. At this time, participants were correct $74.9 \%$ of the time for zero-outcome-associated and $83.9 \%$ for reward-associated faces. In the neutral face condition, positively associated faces were learned faster, differing from neutral faces from $38.5 \%$ until $54.3 \%$ of the learning criterion was met. Note however, that in the very beginning, there was an advantage for zero-outcome-associated compared to rewardassociated faces until $13.3 \%$ of the learning criterion was met (see Figure 4.1). Reaction times (RTs) revealed a main effect of the factor Emotion, $F(1,41)=5.647, p=0.022, \eta_{p}^{2}=$ 0.121 , with faster reaction times for happy compared to neutral facial expressions, and the factor Outcome, $F(1,41)=11.347, p=0.002, \eta_{p}^{2}=0.217$, with faster reaction times for reward- in comparison to zero-outcome-associated faces; an interaction effect was absent. Mean reaction times per experimental condition are summarized in Table 4.1.

\section{ERPs to Target Faces}

A main effect of the factor Expression was revealed on the N170 component, $F(1,41)=$ 14.855, $p<0.001, \eta_{p}^{2}=0.266$, with enhanced negative amplitudes for happy compared to neutral expressions (see Figure 4.2, panel A). This main effect of Expression was also found on the EPN component, $F(1,41)=42.405, p<0.001, \eta_{p}^{2}=0.508$, reflecting larger posterior negativities for happy in comparison to neutral expressions (see Figure 4.2). Modulations

Table 4.1: Mean reaction times in ms and accuracy in \% for learning and consolidation phase (SEMs in parentheses), contrasted for all factor levels of Expression and Outcome. Adequate accuracy of each participant during the learning phase was assured by a required learning criterion (48 of the last 50 trials correct).

\begin{tabular}{lllcc}
\hline & Emotion & Outcome & RTs & Accuracy \\
\hline Learning Phase & Happy & Reward & $1485(57)$ & - \\
& & Zero Outcome & $1608(61)$ & - \\
\cline { 2 - 5 } & Neutral & Reward & $1598(64)$ & - \\
& & Zero Outcome & $1646(63)$ & - \\
\hline Consolidation Phase & \multirow{2}{*}{ Happy } & Reward & $942(27)$ & $99.3(0.2)$ \\
& & Zero Outcome & $1006(29)$ & $99.1(0.2)$ \\
\cline { 3 - 5 } & Neutral & Reward & $986(25)$ & $98.8(0.3)$ \\
& & Zero Outcome & $1016(28)$ & $99.1(0.3)$ \\
\hline
\end{tabular}




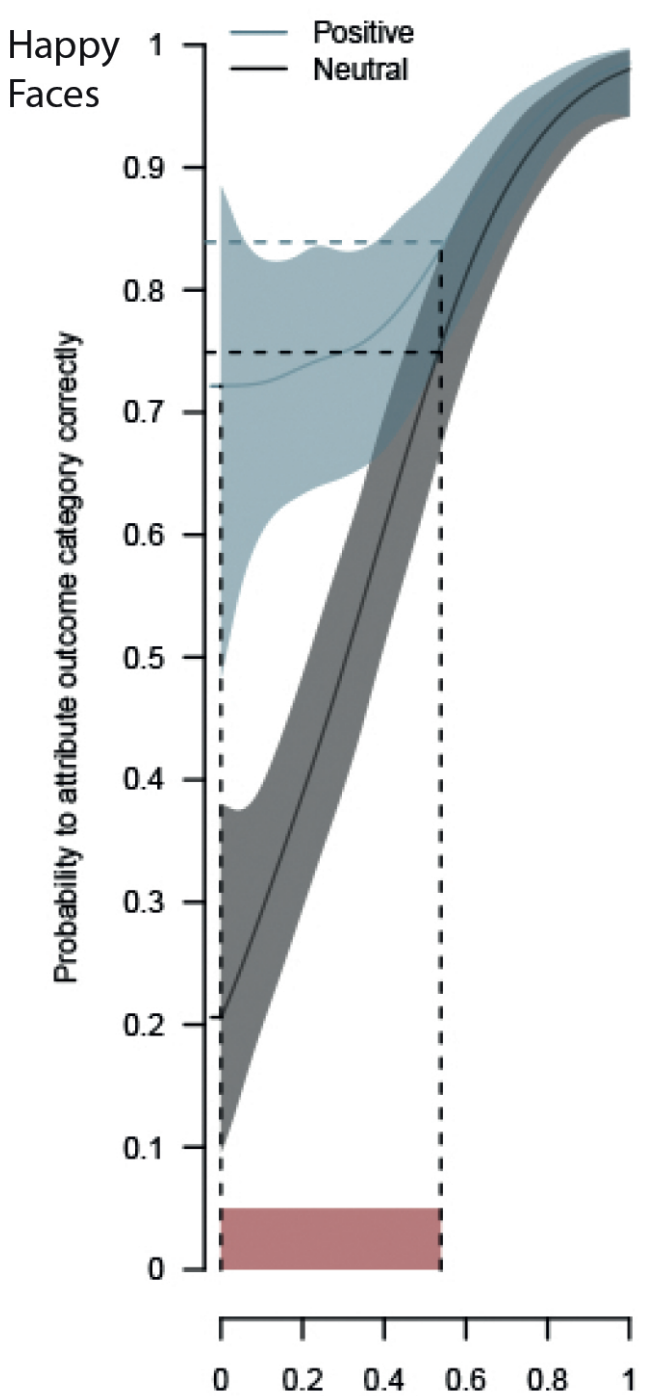

Proportional time until learning criterion

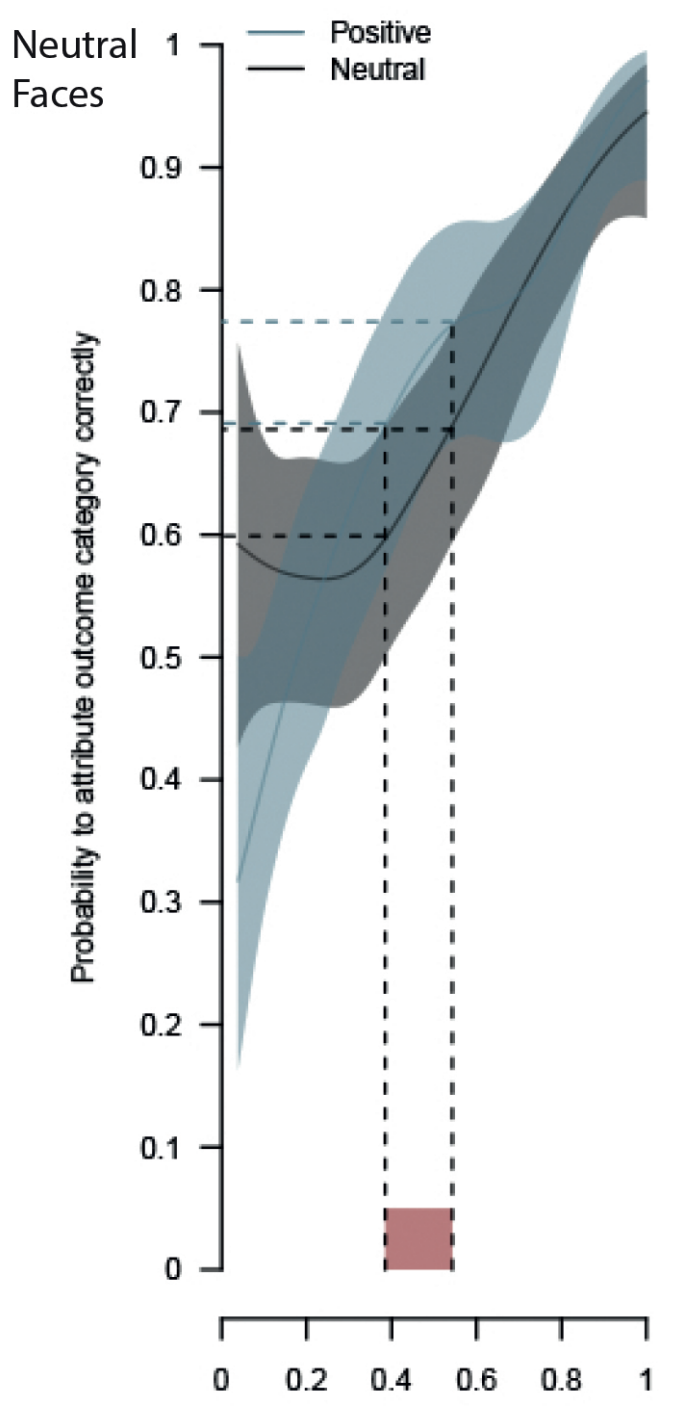

Proportional time until learning criterion

Figure 4.1: Posteriori mean probabilities to attribute the outcome category correctly during the learning phase (illustrated by horizontal dashed lines) at the lower and upper bounds of the time intervals until the learning criterion was met (illustrated by red areas).

of P1 and LPC components by the factors Expression or Outcome were absent. No evidence was revealed for interaction effects on all ERP components of interest. 

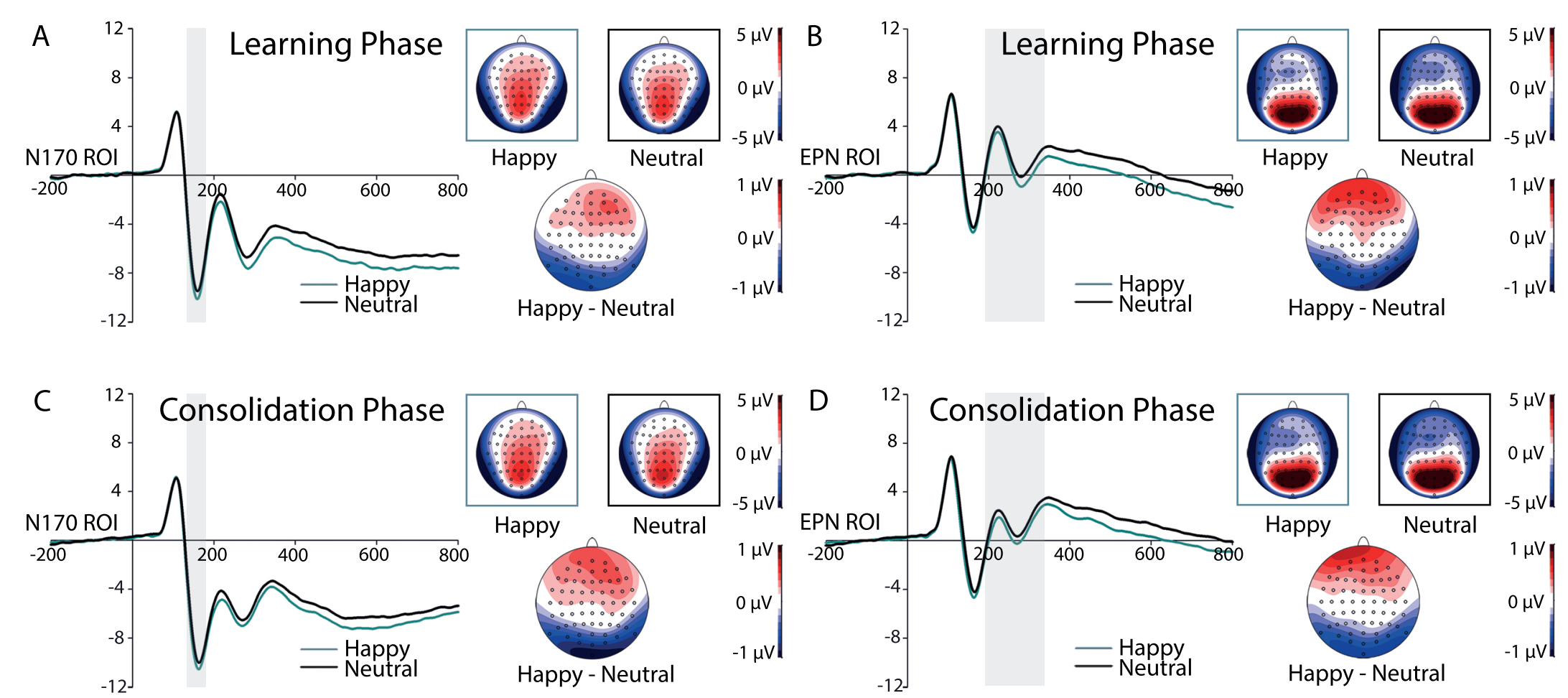

Figure 4.2: Grand-averaged ERPs at N170-ROI electrodes for happy and neutral faces during the learning (A) and consolidation phase (C) with corresponding scalp distributions and topographies of ERP differences between indicated emotion categories. Grand-averaged ERPs at EPN-ROI electrodes for happy and neutral faces during the learning (B) and consolidation phase (D) with corresponding scalp distributions and topographies of ERP differences between indicated emotion categories. Highlighted areas display the time windows of ERP analyses. 


\section{Consolidation Phase}

\section{Behavioral Data}

Accuracy was at ceiling for all conditions $(M s=98.8-99.2 \%)$ during the consolidation phase and did not differ in terms of the factors Emotion and Outcome, $F s(1,41)<1$. RTs of the consolidation phase showed a main effect of the Factor Outcome, $F(1,41)=17.235$, $p<0.001, \eta_{p}^{2}=0.296$, with faster reaction times for reward- compared to zero-outcome associated faces. A main effect of the factor Emotion and an interaction were absent. Mean reaction time and accuracy values per experimental condition are summarized in Table 4.1.

\section{ERPs to Target Faces}

A main effect of the factor Expression was revealed for happy in comparison with neutral facial expressions on the N170 component, $F(1,41)=21.015, p<0.001, \eta_{p}^{2}=0.339$. A main effect for the factor Expression was shown on the EPN component with enhanced negativities for happy compared to neutral facial expressions, $F(1,41)=15.923, p<0.001$, $\eta_{p}^{2}=0.280$ (see Figure 4.2). Furthermore, a main effect of the factor Outcome was found on the LPC component with boosted amplitudes for reward- compared to zero-outcomeassociated faces, $F(1,41)=5.260, p=0.027, \eta_{p}^{2}=0.114$ (see Figure 4.3). P1 component was not modulated by factors Expression and Outcome. Interactions between the factors Expression and Outcome were absent on all components.

\section{Mood}

Participants' mood (according to MDBF) was significantly better after the associative learning task, $F(1,41)=9.718, p=0.003, \eta_{p}^{2}=0.192$, whereas alertness was reduced compared to the beginning of the experiment, $F(1,41)=16.034, p<0.001, \eta_{p}^{2}=0.281$.

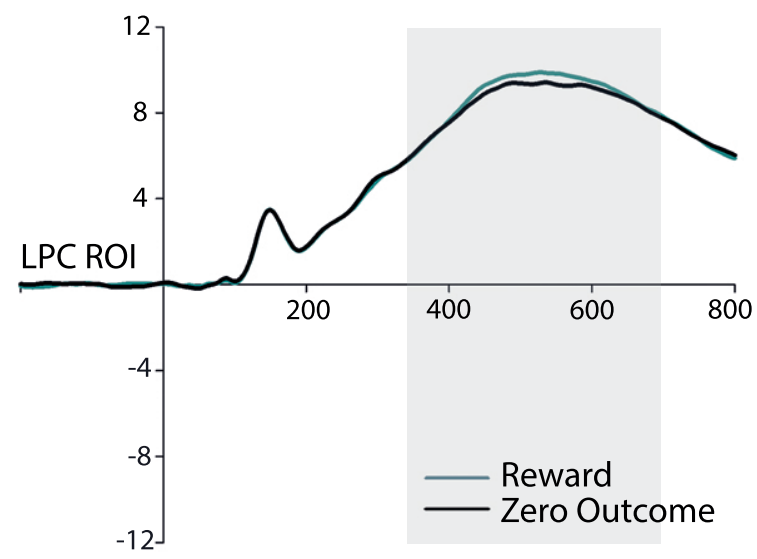

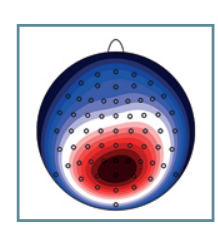

Reward

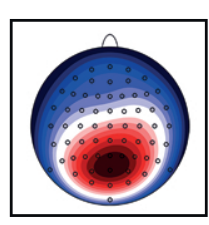

Zero Outcome

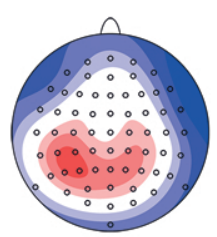

Reward - Zero Outcome
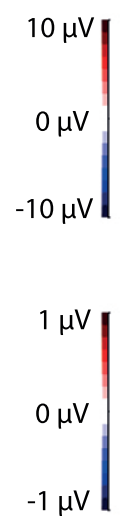

Figure 4.3: Grand-averaged ERPs at LPC-ROI electrodes in response to reward- and neutral-associated faces during the consolation phase with corresponding scalp distributions (left panel) and topographies of ERP differences (right panel) between indicated motivation categories. Highlighted area displays the time windows of ERP analysis. 
The task did not impact participants' calmness.

\subsection{Discussion}

The aim of the present study was the investigation of a potential integration of inherent emotional and associated motivational salience, as, due to the value-driven attention mechanism proposed by B. A. Anderson (2013), their processing should share similar or even common processing characteristics. To this aim, happy and neutral faces were associated with monetary gain or zero outcome via explicit associative learning. The experiment was divided into a learning and consolidation phase to investigate ERPs during and after successful learning. On average, outcome associations had no impact on visual processing in the learning phase, whereas in the consolidation phase, LPC amplitudes, referred to an elaborative processing of relevant stimuli, were enhanced by reward associations. The amplified LPC to reward-associated neutral faces replicates findings of recent similarly designed studies, which could show such effects after implicit reward learning (Hammerschmidt, Kagan, et al., 2017) and associations of reward to (neutral) letters from unfamiliar alphabets (Rossi et al., 2017; Schacht et al., 2012). Importantly, the learning data corroborate our finding of a reward-driven LPC irrespective of the facial expression. Associations of happy faces with reward were learned the fastest, potentially due to an advantage of congruency of expression and outcome valence. However, neutral faces associated with neutral outcome were learned better in the beginning but were outperformed by reward associations during learning. Across both phases, an advantageous effect of reward was further evident in the reaction times, as responses to reward-associated faces were faster than those to faces associated with no outcome. Interestingly, the effect of facial expressions with shorter reaction times for happy than neutral faces was limited to the learning phase, but vanished during consolidation. Although the task was demanding, as indicated by a decrease of participants' alertness, mood increased, presumably due to the overall gain of monetary reward. Together, these findings highlight the increased behavioral relevance of reward associations. Early P1 modulations were expected to be elicited by reward associations (Hammerschmidt, Sennhenn-Reulen, \& Schacht, 2017). For their absence three explanations might be considered: First, a special feature of the present experiment was the restriction to happy and neutral faces as target stimuli on the one hand and gain and zero outcomes on the other hand, leading to the complete absence of any aversive stimulus. One might assume that effects of reward are stronger or even limited to conditions when a negative counterpart (e.g. angry face or monetary loss) is present, while participants in our study have primarily been rewarded. Second, in contrast to our previous study (Hammerschmidt, Sennhenn-Reulen, \& Schacht, 2017), no delay between learning and subsequent testing was implemented. The consolidation of emotional or rather arousing stimuli has been suggested to require time (Sharot et al., 2004), however, also P3 effects modulated by monetary reward were found without overnight consolidation suggesting that this is not mandatory for the occurrence of reward associations (Rossi et al., 2017). Third, similar studies (Bayer et al., 2017; Kulke et al., 2017; Schacht et al., 2012) used a different task during delayed testing, while in the present study, the categorization task 
remained the same and the task relevance of the stimuli did not change throughout the experiment. Therefore, the experimental design, e.g. the task, might play a crucial role in understanding impacts of associated motivational salience and need to be spotlighted in further research. Happy expressions impacted the face-sensitive N170 and the typical emotion-related EPN component both during learning and consolidation. The N170 reflects the configural encoding of a facial stimulus. There is still an ongoing debate whether this process might be impacted by facial expressions of emotion (for reviews, see Hinojosa et al., 2015; Rellecke et al., 2013). However, several studies could demonstrate that the N170 component might be modulated by happy facial expressions (e.g., Bublatzky et al., 2014; Marinkovic \& Halgren, 1998). The emotion-related EPN component was modulated by happy facial expressions. This finding is in line with the conventional link of the EPN to an enhanced encoding of sensory information (Rellecke et al., 2011, 2012; Schacht \& Sommer, 2009a) that occurs independent of context and task demands. Interestingly, as can be seen in Figure 4.2, the difference distributions of the N170 resembled those of the EPN, indicating a potential overlap of these two ERP components (Rellecke et al., 2011; Schacht \& Sommer, 2009a). Future research is needed to fully dissociate these two prominent ERP components and their potential modulations by emotional aspects. Importantly, no interaction of the factors Emotion and Outcome was detected, neither on the behavioral level nor in any of the ERP components of interest, indicating that no integration of the two sources of salience takes place. This absence of interaction effects corroborates studies on emotional words (Kaltwasser et al., 2013) and behavioral findings for faces in a study, where the emotional expression was not task-relevant (Wei \& Kang, 2014). A decrease of the preferential processing of angry (but not happy) faces was previously demonstrated in modulations of the N2pc (Yao et al., 2014), a component linked to spatial attention (Kiss et al., 2008). Together, the results of the present study only partially support the value-driven attention mechanism proposed by B. A. Anderson (2013). According to this assumption, the prioritized processing of effects of associated motivational and inherent emotional salience should be highly similar. In order to investigate whether both types of salience share common processing characteristics, they were orthogonally combined in an associative learning paradigm. The present findings however indicate that these two types of salience were not integrated, as reflected by the absence of interaction effects, diverging brain topographies, and the occurrence of effects in different time windows, under the given experimental conditions.

\section{Conclusion}

Enhanced LPC amplitudes for reward associations independent of the facial expressions (happy, neutral) were demonstrated during the consolidation phase. Happy expressions modulated configural and typical emotion-related ERP components (N170, EPN) during both learning and consolidation. In none of the ERP components and neither in the behavioral data, an interaction between associated and inherent salience occurred. Together, the findings of the present study thus provide novel evidence that within the positive valence dimension both types of emotional/motivational salience are processed in an independent 
way.

\section{Funding}

This work was funded by the German Research Foundation (grant \#SCHA1848/1-1 to $\mathrm{AS})$.

\section{Acknowledgments}

We thank Maren Mette for her contribution to data collection and Holger Sennhenn-Reulen for providing the code for behavioral data analysis.

\subsection{Appendix}

\section{Zygomaticus Major activity}

The impact of the presentation of emotional facial expressions was further investigated by measuring electromyography (EMG) of the involuntarily occurring activation of facial muscles (Dimberg, 1982; Dimberg \& Petterson, 2000). The zygomaticus major has been consistently shown to be activated in the observer by the presentation of happy facial expressions (Dimberg, 1982; Dimberg \& Petterson, 2000; Kret et al., 2013; Larsen, Norris, \& Cacioppo, 2003). Sims et al. (2012) could demonstrate that previously reward-associated neutral faces elicited an increased zygomaticus response when presented with a happy expression compared to novel identities expressing happiness (without previous associations). In line with literature, an increased zygomaticus response was expected for happy compared to neutral facial expressions (e.g., Kret et al., 2013). According to Sims et al. (2012), reward associated happy facial expressions might even boost this activity. Two external
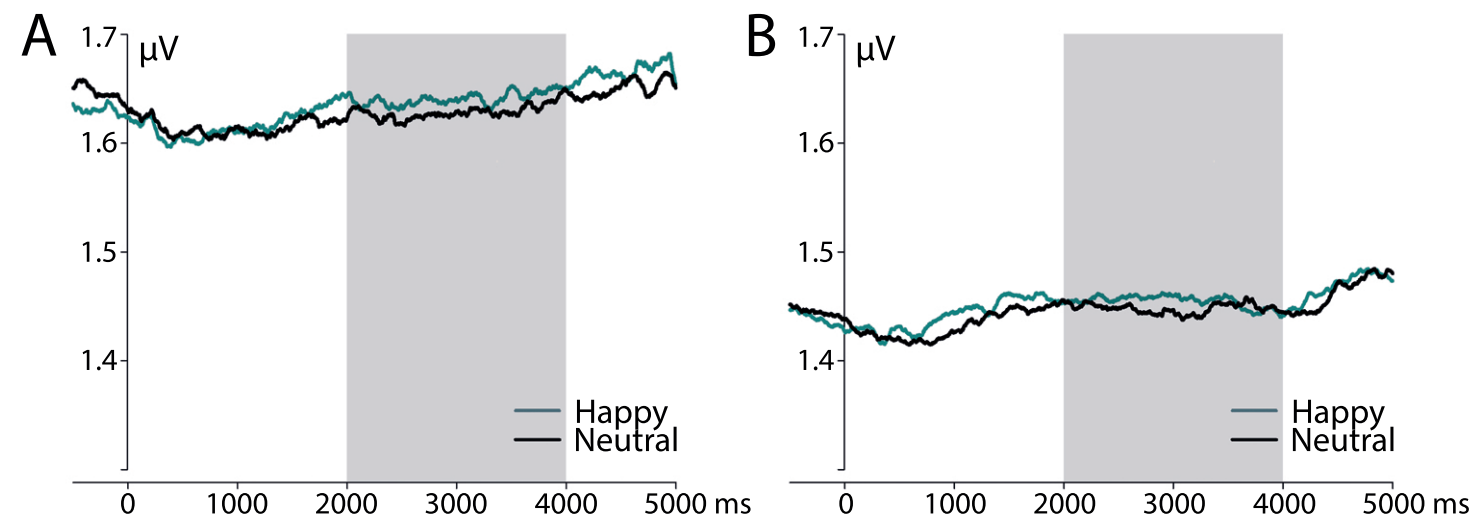

Figure 4.4: Zygomaticus major activity during A: the learning phase and B: the consolidation phase in response to happy and neutral expressions. Highlighted areas display the time window of analysis.

electrodes were placed on the left zygomaticus major (according to Dimberg \& Petterson, 2000; Fridlund \& Cacioppo, 1986). The zygomaticus major signal was offline filtered with a Low Cutoff $(20 \mathrm{~Hz})$ and a High Cutoff $(400 \mathrm{~Hz})$, pooled, and a moving average 
was applied. The continuous EMG signal was segmented into epochs of $7000 \mathrm{~ms}$, starting $2000 \mathrm{~ms}$ before stimulus onset and referred to a $500 \mathrm{~ms}$ pre-stimulus baseline. Based on previous research (Sims et al., 2012), the time window for EMG analysis was chosen from 2000 to $4000 \mathrm{~ms}$ after stimulus onset. During the learning phase (see Figure 4.4, panel A), the zygomaticus major activity was neither modulated by the factor emotion, nor by the factor outcome; an interaction effect was also absent, $F s<2.510$, $p s>0.121$. Zygomaticus major activity decreased during the consolidation phase (see, Figure 4.4, panel B), and no impacts of the factors emotion, outcome or their interaction were detected, Fs $<1.722$, $p s>0.197$. As effects on the zygomaticus major response were absent, future research should investigate whether the experimental design needs to include an aversive condition as counterpart (e.g., angry faces, monetary loss) to elicit an increase in the zygomaticus major activity, not only to happy expressions, but also to happy expressions associated with monetary reward.

\section{Feedback-related ERP components}

The visual processing of feedback-related stimuli was further investigated (for a review, see San Martín, 2012). The feedback-related negativity (FRN) is typically enhanced for loss- compared to reward-related feedback (e.g., Gehring \& Willoughby, 2002), whereas the
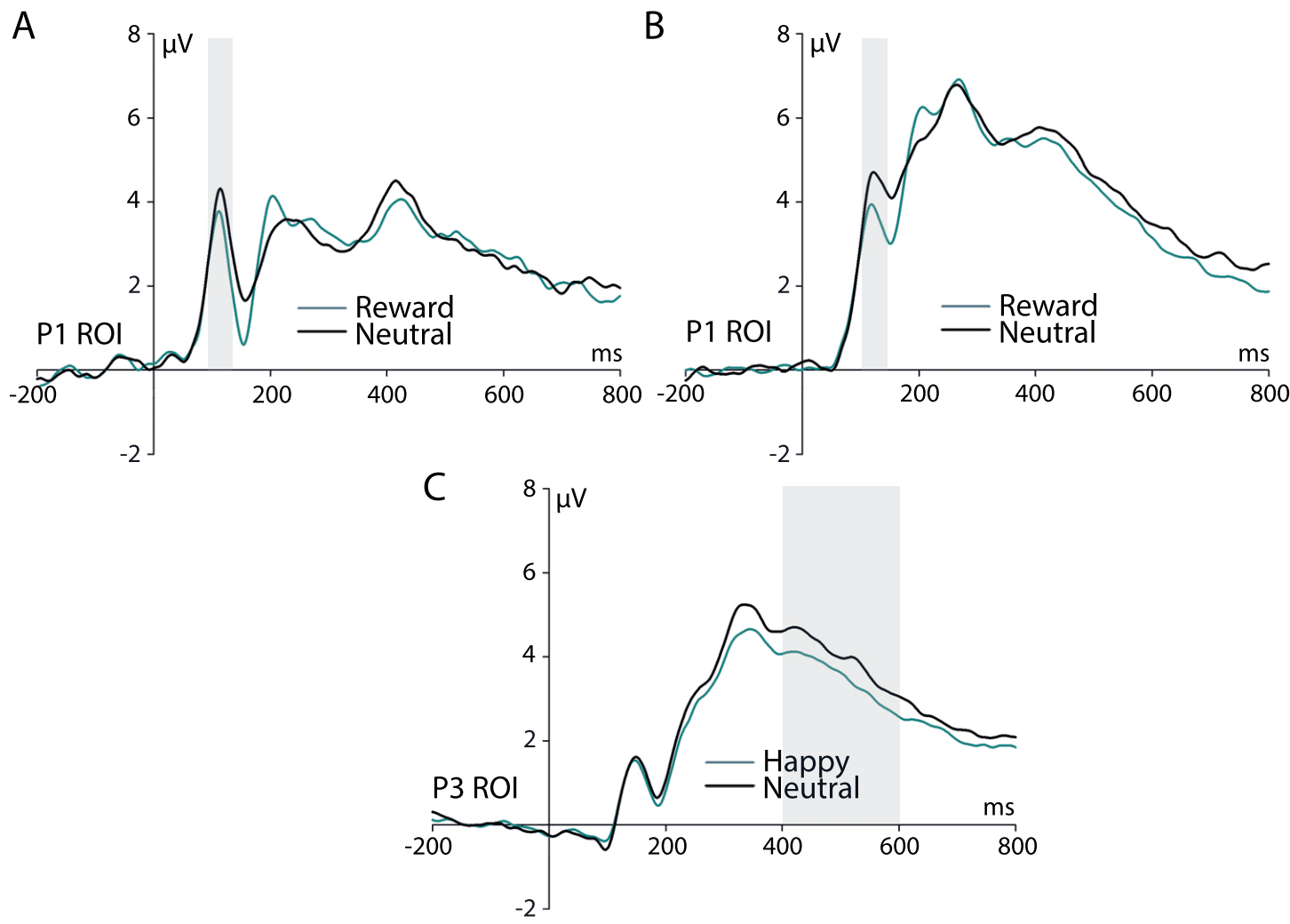

Figure 4.5: Feedback-related ERP modulations for the P1 during the (A) learning and (B) consolidation phase. The feedback-P3 during the (C) learning phase modulated by emotional context. Highlighted areas display the time window of analysis. 
feedback-P3 is demonstrated to be mainly modulated by positive feedback (e.g., Pfabigan, Alexopoulos, Bauer, \& Sailer, 2011). Recently, it was shown that also emotional context - provided by prior presented facial expressions of emotion, can modulate the subsequent feedback processing (Liu, Hsieh, Hsu, \& Lai, 2015). As the visually evoked P1 is known to be sensitive to physical stimulus features (Bayer et al., 2012; Pfabigan, Sailer, \& Lamm, 2015), P1 amplitudes elicited by feedback stimuli might provide further insights in attentional processes (Luck et al., 2000), when feedback stimuli are perceptually identical. However, evidence for P1 modulations elicited by feedback stimuli is, to date, absent. Since all physical features of feedback stimuli, except color-condition assignments, were matched, we were able to test potential P1 modulations. For feedback stimuli, FRN and feedback-P3 modulations were expected (Walentowska, Moors, Paul, \& Pourtois, 2016), driven by reward-indicating feedback. Potential P1 modulations might be impacted by reward- compared to zero outcome-indicating feedback stimuli. Segments were averaged per Subject, Phase (2, learning, consolidation), Expression (2, happy, neutral) and Outcome (2, reward, neutral). Time windows and regions of interest (ROIs) electrodes for feedback-related ERP components were chosen as follows: i) P1: 100-150 ms, O1 and O2 (Pfabigan et al., 2015); ii) FRN: 250-300 ms, Fz and FCz; iii) feedback-P3: 400-600 ms, Pz, P1, P2, CPz (Walentowska et al., 2016). P1 components were quantified as the most positive peak (with $\mathrm{O} 2$ as reference electrode), FRN and feedback-P3 were quantified as mean amplitudes. During the learning phase, a main effect of the factor Outcome was revealed for the P1, $F(1,41)=4.583, p=0.038, \eta_{p}^{2}=0.101$, with higher amplitudes for neutralcompared to reward-related feedback (see Figure 4.5, panel A). The feedback-P3 component was modulated by the factor Emotion, $F(1,41)=10.970, p=0.002, \eta_{p}^{2}=0.211$, with enhanced amplitudes for neutral compared to happy expressions (see Figure 4.5, panel C). FRN modulations and interaction effects on all ERP components of interest were absent during the learning phase. During the consolidation phase, a significant effect of Outcome was detected for the P1 component with amplified peaks for neutral- in comparison to reward-related feedback, $F(1,41)=5.981, p=0.019, \eta_{p}^{2}=0.127$ (see Figure 4.5, panel B). The FRN and feedback-P3 component were not impacted by the factors Emotion and Outcome; interaction effects were absent on all components of interest. Although learning is known to lead to a more efficient processing, resulting in a decrease of the impacts of feedback symbols (Sailer, Fischmeister, \& Bauer, 2010), typical feedback-related ERP modulations (FRN, P3) elicited by outcome were absent both, during learning and consolidation. As the FRN component was shown to be more sensitive for unexpected feedback (e.g., Hajcak, Moser, Holroyd, \& Simons, 2007), the absent effects might be caused by the experimental design, as learning of the salient reward associations was faster leading to an advantage of expected feedback, which also holds true for consolidation, where accuracy was at ceiling. Impacts of outcome were detected on the perceptual level, driven by neutral outcome, whereas effects of emotional context were restricted to P3 modulations driven by neutral expressions during the learning phase. It might be argued that the neutral outcome turned into a negatively interpreted outcome - due to the missing of an aversive condition. This leads to the assumption that loss might be needed to modulate ERPs 
elicited by feedback symbols, supported by the present results, which were driven by the neutral categories. Further research will be mandatory to investigate impacts of feedback on the P1 component and on different learning phases. Interestingly, no interaction of emotional context and outcome-related feedback occurred, supporting the assumption of an independent processing of these two types of salience. 


\section{Chapter 5}

\section{General Discussion}

The aim of the present $\mathrm{PhD}$ project was to investigate the dissociation of neural mechanisms of motivational salience associated to human faces from those mechanisms of inherent facial expressions of emotion. To this aim, three studies were conducted: In Study 1, neutral facial expressions were associated with monetary gain, loss or zero outcome during a learning session; a required learning criterion had to be fulfilled by participants to take part in the test session. ERPs were recorded during delayed testing, while previously associated neutral faces were presented together with familiarized and novel identities expressing emotions (happy, angry, and neutral). The processing of inherent emotional expressions of novel identities was sustained from perceptual to higher-order processing, reflected in enhanced amplitudes of the $P 1, N 170, E P N$ and $L P C$ components, and were mainly driven by angry facial expressions. In contrast, previously reward-associated neutral faces were shown to modulate the early perceptual processing reflected in enhanced P1 amplitudes. As associated motivational salience did not impact the subsequent processing stages after the perceptual processing, the findings indicated that these associations did not mandatorily result in a sustained preferential processing, as demonstrated for facial expressions of emotion. Study 2 investigated whether associated motivational salience can be acquired when associations with a monetary outcome are learned implicitly and whether these ERP effects resemble robust effects elicited by facial expressions of emotion. To this aim during a learning session - neutral facial expressions were implicitly associated with reward, loss, or zero outcome via outcome-indicating cues, presented at the beginning of every trial. A face-matching task including a subliminal prime assured performance on chance level and an equalization of performance-dependent reward, loss, or zero outcome conditions. During delayed testing, previously associated neutral faces were presented together with facial expressions of emotion while the face-matching task remained the same, however, without the presentation of a motivational cue and performance feedback. Pupil dilations were recorded in addition to ERPs during the learning and the delayed test session. During learning, reward- and loss-related cues were not only shown to elicit a prioritized processing themselves, but also to impact subsequent (even task-irrelevant) processing stages. However, effects of the implicitly associated motivational context on neutral target faces were absent. Further, reward- and loss- indicating cues elicited an increase in pupil size compared to neutral-indicating cues. During delayed testing, previ- 
ously reward-associated neutral faces elicited sustained centro-parietal positivities. Facial expressions of emotion (happy and angry) modulated the emotion-related EPN component. The results demonstrated that neutral stimuli might gain increased salience via implicit learning. Furthermore, an advantage for reward over loss associations was detected. Study 3 aimed to investigate the potential integration of associated motivational and inherent emotional salience. In this study, happy as well as neutral expressions were associated with reward or zero outcome during a learning and a consolidation phase. The experimental design was similar to Study 1. A categorization task, to judge whether a face is reward or zero outcome-related, was implemented during both learning and consolidation phase. The learning phases ended when participants reached the required learning criterion. It was demonstrated that happy facial expressions modulated the N170 and the EPN component during the learning and consolidation phase. For reward- compared to zero outcome-associated faces, enhanced LPC amplitudes were found during the consolidation phase. The results of Study 3 suggested that impacts of inherent emotional and associated motivational salience are not integrated. This was indicated by the absence of interaction effects, different time windows and diverging brain topographies.

In the following, the results of these three studies will be discussed within a broader context.

\section{Effects of inherent emotional salience on ERP components}

The impacts of inherent emotional salience on emotion-related ERP components are typically investigated through the presentation of emotional stimuli such as words, pictures or faces (e.g., Bayer \& Schacht, 2014). Faces are particularly salient stimuli (e.g., immediate indicators of affective dispositions in others) and, therefore, of special interest for investigating emotion-related ERP modulations. In the present thesis, facial expressions of emotion (happy, angry and neutral) were anticipated to elicit typical emotion-related ERP modulations, as they were consistently reported to impact visual processing on successive stages from the P1 component to the N170, EPN and LPC component (e.g., Rellecke et al., 2011, 2012).

P1 amplitudes were enhanced for inherently angry facial expressions in Study 1, suggesting that emotion-related ERP modulations already started on the perceptual level. The early visual processing of emotional, especially threat-related, facial expressions was argued to be coarse and rapid (Rellecke et al., 2011), predominantly instantiated by the magnocellular pathway (for reviews, see Vuilleumier, 2005; Vuilleumier \& Pourtois, 2007). This pathway allows attention to be rapidly directed to these relevant stimuli, presumably due to initial top-down effects on the visual cortex (Vuilleumier \& Pourtois, 2007). Note that in Study 1, a simple gender decision task was used. In contrast, P1 modulations on the target faces in Study 2, seem to have been suppressed by task difficulty, as a face-matching task with a subliminal prime was used. This indicates that the elicitation of P1 modulations might be dependent on task demands. Pratt et al. (2011) reported a suppression of P1 modulations with a dual task paradigm. The authors suggested that with an increase of task demands and a high working memory load, P1 modulations might be suppressed, as 
attentional resources are limited. The lack of P1 modulations in Study 3 was, however, in line with previous research, as it was shown that P1 effects were mainly observed for threatrelated facial expressions (e.g., Pourtois et al., 2004; Rellecke et al., 2012; Vuilleumier \& Pourtois, 2007), and no angry, but only happy and neutral expressions were presented.

Regarding the subsequent N170 component, there is an ongoing debate, whether this face-sensitive component is modulated by facial expressions of emotion (for reviews, see Hinojosa et al., 2015; Rellecke et al., 2013). Several studies reported that the N170 was unaffected by facial expressions (e.g., Eimer, Holmes, \& McGlone, 2003) and supported the assumption of an influential face recognition model that the processing of configural features and emotional expressions is independent (Bruce \& Young, 1986). On the contrary, enhanced N170 amplitudes were found for facial expressions of emotion compared to neutral expressions (e.g., Rellecke et al., 2012; Valdés-Conroy, Aguado, Fernández-Cahill, Romero-Ferreiro, \& Diéguez-Risco, 2014), suggesting that configural and emotional features might be processed simultaneously. In all three present studies, enhanced N170 amplitudes elicited by facial expressions of emotion (angry expressions in Study 1 and 2, and happy expressions in Study 3) were detected. This suggests that the N170 component is modulated by facial expressions of emotion, independent of task demands. However, it is still unclear, which specific task properties might lead to these N170 modulations. Therefore, one important focus for future research should be to understand the potential interaction of the configural encoding and the emotional expression of a face.

In line with previous research (e.g., Bublatzky et al., 2014; Junghöfer et al., 2001; Rellecke et al., 2011, 2012; Schacht \& Sommer, 2009a; Schupp et al., 2004), the typical emotion-related EPN component was modulated in all three studies, with enhanced negativities for both angry (Study 1 and 2) and happy (Study 1 to 3) expressions. These EPN modulations occurred irrespective of conflicting information of the familiarized identities with emotional expressions presented in Study 1, and the high cognitive load of the task in Study 2. In Study 3, enlarged posterior negativities were detected for happy compared to neutral expressions, both during learning and consolidation. Together, these findings strongly support the assumption that the EPN reflects an automatic and task-independent sensory encoding elicited by stimuli carrying inherent emotional salience (Rellecke et al., 2011).

In addition, in Study 1, enhanced LPC amplitudes were demonstrated for angry facial expressions, replicating findings of previous research (e.g., Rellecke et al., 2012; Schupp et al., 2004). In Study 2, however, LPC modulations elicited by facial expressions of emotion were absent. In line with previous studies (e.g., Rellecke et al., 2012), the prioritized processing of emotional expressions might voluntarily be suppressed when the task is demanding, and the emotional expression is not (task-) relevant in a given situation. In Study 3, the facial expressions presented in the experiment were restricted to happy and neutral expressions. As the LPC has been referred to higher-order evaluations especially elicited by angry facial expressions, the absence of an LPC effect elicited by happy facial expressions is supported by previous research (e.g., Schupp et al., 2004).

In summary, facial expressions of emotion impacted subsequent processing stages, start- 
ing at the perceptual level (P1), to configural (N170) and sensory encoding (EPN) up to higher-order evaluations (LPC). Continuous emotion-related modulations occurred on all ERP components of interest in Study 1, whereas impacts of facial expressions of emotion were only reflected on the EPN component in Study 2 and 3, potentially caused by variations in experimental designs and task demands. Successive processing stages were impacted by angry facial expressions, potentially induced by a frequently reported processing bias toward threat-related (angry) stimuli (for more details, see Schupp et al., 2004; Vuilleumier \& Pourtois, 2007). The EPN, referred to sensory encoding of a visual stimulus (e.g., Rellecke et al., 2011), was modulated by both inherently happy and angry facial expressions. In the last years of research, various researchers aimed to answer how continuous effects across different processing stages, elicited by facial expressions of emotion, might be enabled. For instance, Pourtois et al. (2013) argued that the amygdala might play a central role to provide not only a rapid feedback to the visual cortex (striate and extrastriate) after the presentation of an emotional stimulus, but also enables impacts on later, memory-associated processing stages. The authors suggested that an interplay of several brain areas might be involved (e.g., anterior cingulate cortex; ACC) and, thus, further evidence needs to be provided to determine the exact neural pathways.

\section{Effects of associated motivational salience on ERP components}

According to the postulation of a value-driven attention mechanism (B. A. Anderson, 2013) and the theoretical framework of motivated attention (Lang, Bradley, Cuthbert, et al., 1997), a prioritized neural processing was not only expected for faces carrying inherent emotional salience, but also for neutral faces associated with motivational salience. The effects detected in the present studies can be considered as driven by associated motivational salience, since stimuli were controlled for physical features (e.g., luminance), and completely counterbalanced across conditions to avoid confounding due to potential stimulus-feature effects.

In Study 1, previously reward-associated neutral faces were found to elicit enhanced P1 peak amplitudes compared to neutral outcome associations during delayed testing, reflecting the impact of associated motivational salience on sensory processing. This rapid prioritization allows to direct attention to the most relevant stimuli (e.g., Pourtois et al., 2004), which might be explained by an automatic encoding of reward (Krebs, Boehler, Egner, \& Woldorff, 2011). Recently, it was demonstrated that associated motivational salience might even impact responses of the visual areas, specifically in V1 (Rossi et al., 2017) and V4 (Bourgeois, Chelazzi, \& Vuilleumier, 2016). In order to explain how knowledge from associative learning might impact face processing, two independent parallel routes were suggested to be rapidly activated: one route through the anterior system and the other through face-sensitive brain areas (Gamond et al., 2011). An integration of these two routes is suggested to occur around $150 \mathrm{~ms}$ after stimulus onset, linking prior knowledge and the processing of a facial expression. While in Study 1, the perceptual processing of neutral faces was impacted by previous reward associations, augmented LPC amplitudes for associated reward were detected in Study 2 and 3. The LPC component has been 
referred to the voluntary direction of attentional resources (including working memory), to enable an elaborate processing of relevant stimuli (e.g., Nieuwenhuis et al., 2005; Schupp, Markus, Weike, \& Hamm, 2003). LPC modulations have been already observed for previous reward-associated unknown letters (Rossi et al., 2017; Schacht et al., 2012), however, our studies (Study 2 and 3) are the first to provide evidence that reward-association can also affect the LPC in response to human faces.

Interestingly, effects of associated motivational salience demonstrated in the three present studies were only driven by reward associations, while impacts of monetary loss were absent. One reason might be that in explicit associative learning paradigms, which are typically employed to investigate effects of associated motivational salience, the amount of reward increases, whereas the amount of loss decreases due to successful learning. Therefore, the P1 effect, driven by neutral faces previously associated with monetary reward (demonstrated in Study 1) cannot be interpreted as a distinct reward advantage. By implementing the associative learning paradigm within our study design, the monetary outcome was not balanced, as successful learning implied an increase of outcome (participants won 20 cents for correctly categorizing reward-related neutral faces and lost 10 cents for correctly classifying loss-related faces). In contrast, the experimental design employed in Study 2 (a face-matching task with a subliminal prime) had not only the advantage of enabling the investigation of effects of implicitly associated motivational salience, but also guaranteed a balanced outcome of monetary reward and loss during the learning session. As task performance was at chance level, an equalization of performance-dependent reward, loss, or zero outcome conditions was assured. An advantage of reward over loss was reflected on LPC modulations elicited by the motivational cue (reward-, loss-, or zero outcome-indicating) during the learning session. Even when the amount of money won and lost was held equal, effects of associated motivational salience during delayed testing revealed a clear advantage of reward over loss associations in and even before the LPC time window (starting around $200 \mathrm{~ms}$ and lasting until $700 \mathrm{~ms}$ after stimulus onset), linked to a sustained higher-cognitive processing. This suggests that the reward advantage, previously detected during the learning session, was constant after overnight consolidation. In Study 3, reward associations also revealed enhanced LPC amplitudes compared to zero outcomeassociations, however, no loss condition was present, which narrows the contribution of Study 3 regarding the existence of a reward advantage over loss. Overall, the ERP results of the three studies indicated that monetary reward looms over monetary loss. These ERP modulations were further supported by the analyses of explicit learning behavior in Study 1 and 3. It was shown that reward associations were learned better and faster compared to loss (Study 1) and neutral outcome (Study 1 and 3), replicating effects of Rossi et al. (2017). Chapman et al. (2015) demonstrated that reward-associated targets were processed faster than loss-associated targets. The authors argued, that the inhibition necessary for loss aversion takes more time than the facilitated processing elicited by reward associations. Recently, Barbaro et al. (2017) systematically investigated whether reward elicits a prioritized processing compared to loss in a fMRI study. They could demonstrate that human visual selection is not economically normative even as reward associations are prioritized, 
although the gain of reward and the avoidance of loss should be equivalently beneficial. These findings seem to challenge the assumptions of the well-established prospect theory (Kahneman \& Tversky, 1979; Tversky \& Kahneman, 1992) that suggested higher impacts of monetary loss in decisions under risk (Tom, Fox, Trepel, \& Poldrack, 2007; Trepel et al., 2005), especially during reinforcement learning tasks (Kim et al., 2015; Wächter et al., 2009). Thus, the reward prioritization and the accompanied absence of effects driven by monetary loss, demonstrated in the present studies, is in line with recent research (Barbaro et al., 2017; Chapman et al., 2015). Together, the present studies provide evidence that motivational salience associated to human faces impacts either the early perceptual (Study 1) or later higher-order evaluation stages (Study 2 and 3) and further, that both explicit (Study 1 and 3; e.g., Rossi et al., 2017; Schacht et al., 2012) and implicit (Study 3; e.g., Bourgeois, Chelazzi, \& Vuilleumier, 2016) associative learning of motivational salience is shaping attentional processes.

\section{Same or different? The dissociation of associated motivational and in- herent emotional salience}

In the following, the neural processing similarities and differences, revealed in the current series of experiments, of associated motivational and inherent emotional salience on human face processing will be discussed.

\section{Similarities}

The present findings support the existence of a value-driven attention mechanism (B. A. Anderson, 2013) assuming that stimuli associated with motivational and stimuli carrying inherently emotional salience share distinct similarities in their neural processing. In the present studies, it could be demonstrated that the P1 and the LPC component seem to be sensitive to both, inherent emotional and associated motivational salience. A prioritized processing of relevant stimuli on both, the perceptual level and on more elaborative processing stages was suggested to assure an adequate, goal-directed behavior (Pessoa \& Engelmann, 2010). One might argue that reward- and threat-related stimuli share distinct processing similarities, as the P1 and the LPC component were exclusively modulated by threat-related (angry) facial expressions and neutral faces previously associated with monetary reward. This assumption, based on the present ERP findings, is further supported by results of dipole analysis for the $\mathrm{P} 1$ components elicited by associated motivational and inherent emotional salience in Study 1. As shown, the neural sources for reward-associated neutral and angry expressions were both detectable in the extrastriate visual cortex (Brodman area (BA) 19). Pourtois et al. (2013) argued that the amygdala might provide a rapid feedback to the extrastriate visual cortex, presumably enabling a rapid relevance detection of both emotionally and motivationally relevant stimuli (e.g., B. A. Anderson, Laurent, \& Yantis, 2014; Pourtois et al., 2004; Stolarova et al., 2006). To explain such early effects of associated motivational salience, Krebs et al. (2011) proposed that reward associations are automatically encoded - which was comparably proposed for facial expressions of emotion (Rellecke et al., 2011). The LPC component is typically triggered by angry facial expres- 
sions due to their evolutionary relevance (e.g., Schupp et al., 2004). However, Lang (2010) assumed that reward and threat signals are equally relevant for our well-being and survival as they either stimulate the appetitive or aversive system. Furthermore, the multiple attention gain control (MAGiC) model (Pourtois et al., 2013) proposed that the rapid relevance detection and the subsequent processing of emotionally and motivationally relevant stimuli is a result of the interplay of different brain regions. Especially the amygdala (e.g., Sander, Grafman, \& Zalla, 2003) and the anterior cingulate cortex (ACC; e.g., Hickey et al., 2010; Umemoto, HajiHosseini, Yates, \& Holroyd, 2017) were argued to play a central role, although a multitude of additional brain structures might be involved (for a review, see Pourtois et al., 2013). To ensure the potentially shared mechanisms assumed by the present findings, future studies might aim at combining ERP and fMRI measurements to explore how the temporal sequence of brain activity during the processing of neutral faces previously associated with motivational salience and facial expressions of emotion is summarized in fMRI activation maps.

On a side note, an interesting finding worthy of mention here was revealed in Study 2. Inherently neutral diamond-shaped cues acquired salience while participants explicitly learned, prior to the beginning of the experiment, whether they are reward-, loss-, or zero outcome-indicating. This was reflected in a posterior negativity in the EPN-related time window. Topographical comparisons confirmed that the cue-EPN (driven by reward- and loss-context) resembled the EPN component elicited by either happy or angry expressions during the test session. One could argue that, with explicit knowledge, a motivational cue might gain an emotional meaning as the EPN is typically linked to an enhanced sensory encoding of emotional stimuli (e.g., Rellecke et al., 2011). Evidence for EPN modulations elicited by a motivational cue is, to date, absent. However, a recent study demonstrated that the cue-P3 might be modulated by reward- compared to loss-indicating cues (Zheng et al., 2017). Future research needs to investigate the impacts of motivational context and whether these impacts might be similar to inherent emotional salience on sensory encoding stages.

In summary, the neural processing of motivational salience associated to neutral faces and facial expressions of emotion seem to share distinct similarities as suggested by the value driven-attention mechanism (B. A. Anderson, 2013). Especially inherently angry and reward-associated faces elicited prioritized processing that is further supported by the theoretical framework of motivated attention (Lang, Bradley, Cuthbert, et al., 1997).

\section{Differences}

Although processing similarities were detected, the results of the present studies also provided evidence for differences in the neural processing of associated motivational and inherent emotional salience. Facial expressions of emotion elicited modulations on subsequent ERP components in Study 1, starting on the perceptual level (P1), and sustaining over configural (N170) and sensory encoding (EPN) until higher-order evaluations (LPC). In contrast, reward-associated neutral faces were restricted to enhanced P1 peak amplitudes indicating that associated motivational salience did not lead to a sustained processing. 
In Study 2, effects of inherent emotional salience were restricted to the emotion-related EPN component. Nevertheless, an LPC modulation was revealed for previously rewardassociated neutral faces suggesting that LPC effects were not entirely suppressed by the cognitive load of the face-matching task. Therefore, reward-associated neutral faces might be processed more deeply as they seem to be more relevant in this task than the inherently angry facial expressions. In the service of an elaborative processing (reflected by the LPC component), the previous sensory processing stages were demonstrated to encode the inherent emotional meaning of the given stimuli (e.g., Cuthbert et al., 2000; Olofsson et al., 2008; Schupp et al., 2003). The findings of Study 2 and 3, however, suggest that LPC modulations might occur independent of previous processing stages. The results therefore indicate that associated motivational salience does not impact successive processing stages, as it was demonstrated for faces expressing inherent emotions.

A further indication that the neural processing of emotional and motivational salience might differ was provided by the results of Study 3. Although neutral expressions were learned together with inherently happy expressions, associated motivational salience modulated higher-order evaluations (LPC). However, it was demonstrated that this effect occurred independently of effects elicited by inherent emotional salience (EPN), reflected in different time windows, diverging brain topographies and the absence of interaction effects on all ERP components of interest. However, future research should investigate whether an angry and/or loss condition as negative counterpart is required to replicate effects of associated reward to neutral faces on perceptual processing stages (Study 1). In addition, to assess the potential integration of inherent emotional and associated motivational salience, future studies should investigate this in a systematic manner (i.e., happy, angry and neutral facial expressions associated with monetary reward, loss, or no outcome).

The EPN is suggested to reflect an, presumably automatic (Rellecke et al., 2011), enhanced sensory encoding of emotionally salient stimuli for both positive and negative emotions (e.g., Bublatzky et al., 2014; Junghöfer et al., 2001; Rellecke et al., 2011, 2012; Schacht \& Sommer, 2009a; Schupp et al., 2004). EPN modulations were consistently found for inherently angry (Study 1 and 2) and happy (Study 1 to 3) expressions, while modulations by associated motivational salience were absent in all three studies. Previous research reported EPN modulations not only across varying experimental designs, but also across domains [facial expressions of emotion (e.g., Rellecke et al., 2011, 2012), emotional pictures (e.g., Olofsson et al., 2008) and emotional words (e.g., Kissler et al., 2007; Schacht \& Sommer, 2009b)] and task demands (Rellecke et al., 2012). Therefore, more specifically, the EPN seems to reflect the encoding of an inherent emotional meaning (Kissler, Herbert, Winkler, \& Junghofer, 2009; Palazova, 2014). The results of the present studies therefore indicate that an inherent emotional meaning, as carried by facial expressions of emotion, might not be triggered by associated motivational salience.

To conclude, the results of the present studies indicate that neutral faces associated with motivational salience (especially with monetary reward) elicited a prioritized neural processing independent of slight changes in the experimental design of the three studies. As suggested by the value-driven attention mechanism (B. A. Anderson, 2013), this atten- 
tional prioritization seems to be similar to the preferential processing reported for facial expressions of emotion. Therefore, this finding is in line with the assumptions of the motivated attention model of affect (Lang, Bradley, Cuthbert, et al., 1997) that suggests that motivational and emotional factors are directly linked. However, the results of the present studies do not indicate an entirely equal processing. Facial expressions of inherent emotion (especially angry expressions) modulated subsequent processing stages starting on the perceptual level and sustained over configural (N170) and sensory encoding (EPN) until higher-order evaluations stages (LPC; revealed with an easy task in Study 1). In contrast, faces associated with motivational salience either modulated the perceptual processing ( $\mathrm{P} 1$; Study 1) or higher-order evaluation stages (LPC; Study 2 and 3) while the emotion-related EPN component seems to be insensitive for impacts of associated motivational salience in the present studies, indicating that an inherent emotional meaning might not be triggered by monetary incentives.

The value-driven attention mechanism proposed by B. A. Anderson (2013) was only partially supported by the present results. One the one hand, distinct processing similarities were revealed, as it could be demonstrated that neutral faces associated with motivational salience (especially reward) elicited a prioritized processing on either perceptual or higher-order stages. This finding is comparable to the preferential processing demonstrated for stimuli carrying inherent emotional salience. On the other hand, an integration of effects of associated motivational and inherent emotional salience was absent. Further, continuous ERP modulations impacting several processing stages could not be demonstrated. Thus, as indicated by the present findings, an emotional meaning, carried by inherent facial expressions of emotion, might not be triggered by monetary incentives.

\section{Limitations and Future Research}

Although, within the three studies conducted, neural mechanisms of associated motivational salience were elucidated, several limitations that might have had an impact on the reported results should also be considered. In addition, implications for future research are discussed.

As augmented P1 amplitudes elicited by previously reward-associated neutral faces were detected in Study 1, they were also expected to be found in Study 2 and 3, however, P1 modulations were absent. One factor that might have contributed to these incoherent findings across the studies are the task-specific requirements, already shown by past studies to substantially impact the visual processing of emotionally (e.g., Rellecke et al., 2012; Schacht \& Sommer, 2009a) and motivationally relevant stimuli (e.g., Pessoa \& Engelmann, 2010). For instance, in Study 1, a simple gender decision task (accuracy at ceiling) was employed, whereas in Study 2, a demanding face-matching task with a subliminal prime was implemented. In Study 3, however, a categorization task, similar to the task of the learning session in Study 1, was performed throughout the experiment. During the learning phase, the task was challenging, but solvable (indicated by participants reaching the required learning criterion), whereas during the consolidation phase, the task was easy to fulfil, demonstrated by accuracy at ceiling. Several studies reported that task demands might 
have a direct impact on human face processing, especially reflected on the LPC component (Holmes et al., 2009; Rellecke et al., 2012; Valdés-Conroy et al., 2014; Van Strien, De Sonneville, \& Franken, 2010) but also on perceptual processing stages (P1; Pratt et al., 2011). A systematical comparison of different tasks would be necessary to fully understand the impacts of cognitive load and task demands on the elicitation of ERP components driven by both associated motivational and inherent emotional salience.

Further, one might wonder, as LPC modulations were detected in Study 2 and 3, why they were not present in Study 1. As the LPC component reflects the relevance of given stimuli and the necessity to process them more deeply, one might argue that the reward associations in Study 1 were not relevant enough to elicit LPC modulations. As in Study 3, reward associations (and the resulting possibility to receive more money) were continuously present, reward-associated stimuli should be relevant. However, neither a test session with a different task, nor one without a monetary outcome were conducted, and therefore, results cannot clarify whether the LPC component might still be modulated during delayed testing. In Study 2, however, higher reward magnitudes were used (+50 cents) compared to Study 1 and 3 ( +20 cents for correctly assigned rewards), therefore it needs to be discussed and investigated whether higher amounts of money might boost ERP effects (e.g., Meadows et al., 2016), especially on higher-order evaluation stages (A. Sato et al., 2005; Yeung \& Sanfey, 2004). A higher reward magnitude could also lead to strengthened ERP effects driven by associated motivational salience; however, studies systematically investigating the impacts of reward magnitude were restricted to feedback-related ERP components. One has to bear in mind that the vast majority of participants of the present studies were undergraduates and, therefore, one interesting line for future research is to investigate whether the social status (especially the income) might have an influence on reward/loss associations (Bhanji \& Delgado, 2014). Pointing in the same direction, it has been argued that reward sensitivity is declining with age (for a review, see Eppinger, Hämmerer, \& Li, 2011). As in our studies the majority of participants were of similar age, future studies could investigate how aging affects the neural processing of reward and/or loss associations.

Furthermore, consolidation phases differed in the following aspects between the three studies: In Study 1 and 2, the learning session was followed by overnight consolidation and a test session on the next day, whereas in Study 3, the consolidation phase was recorded directly after reaching the learning criterion in one experimental session. As sleep seems to play a crucial role not only regarding the processing of emotional stimuli (Hu, StylosAllan, \& Walker, 2006; Walker, 2009, for a review), but also on learned associations (Alger \& Payne, 2016; Javadi, Walsh, \& Lewis, 2011), effects of associated motivational salience might differ after overnight consolidation. In Study 1, ERPs were only recorded during delayed testing and in Study 3 directly after learning without overnight consolidation, making it impossible to investigate overnight effects. However, results of Study 2 support the idea of overnight consolidation, as during learning, ERP effects on target faces were absent, whereas after overnight consolidation, target faces were modulated by previously associated reward. However, overnight consolidation might only be required for implicit associative learning, as P3 effects modulated by monetary reward were demon- 
strated during consolidation in an explicit learning paradigm (Rossi et al., 2017). Further, Dunsmoor, Murty, Davachi, and Phelps (2015) suggested that effects of consolidation are not dependent on sleep, but on time. An interesting question emerging from the findings of the present studies is how long effects of acquired salience remain detectable, as evidence seems to be rare. One week after a learning session, Ventura-Bort et al. (2016) detected boosted centro-parietal ERP amplitudes for neutral objects, which were previously paired with emotional context (pleasant and unpleasant background scenes). Della Libera and Chelazzi (2009) failed to find impacts of previous reward associations on neutral shapes after five days while measuring RTs. Therefore, future research is needed to investigate not only impacts of overnight consolidation, but also the stability of effects of associated motivational salience over time (e.g., a week or a month after learning). In addition, to investigate the potentially shared mechanisms of threat and reward and to answer the question whether they are of comparable behavioral relevance (Lang, 2010), future studies could systematically vary the period of consolidation. Mueller and Pizzagalli (2016) could demonstrate that previously fear-conditioned neutral faces elicited prioritized processing, approximately one year after conditioning. Based on this finding, they argued that especially threat-related associations remain stable over time - even without explicit knowledge of the participants. Based on the results of the present studies, future research could not only determine whether the face itself or the learned outcome associations are remembered after a longer period of consolidation, but also whether the presentation of the neutral stimuli associated with motivational salience (especially reward) might still elicit prioritized processing.

In addition, it is not only interesting how long these effects of associated motivational salience remain stable, but also when in time they occur. Rossi et al. (2017) investigated ERP effects of associated motivational salience after a learning session, during subsequent consolidation and delayed testing. They found LPC effects modulated by monetary reward both during consolidation and in the subsequent Old/New task. However, the evolvement of modulations of ERP components in time needs to be spotlighted as they might occur also during learning. The experimental design of Study 2 tried to deal with this knowledge gap by measuring ERPs also during the learning session. It would be of great interest to clarify at which stage of learning the ERP modulations elicited by associated motivational salience evolve, as neural changes might occur before the successful explicit learning takes place.

As shown in Study 2, the impacts of associated monetary reward and loss were not fully symmetric. This finding opens up the question whether a different aversive condition would have led to symmetric impacts on ERPs. In future studies, participants that receive a positive outcome (e.g., money) in the reward condition could also receive a negative outcome in the aversive condition for instance by electric shocks (e.g., Choi, Padmala, \& Pessoa, 2015; Choi, Padmala, Spechler, \& Pessoa, 2014; Heydari \& Holroyd, 2016; Rehbein et al., 2014), aversive tones (e.g., Hintze et al., 2014; Kastner, Pauli, \& Wieser, 2015; McTeague, Gruss, \& Keil, 2015; Mueller, Panitz, Hermann, \& Pizzagalli, 2014) or aversive smells (e.g., Kastner, Flohr, Pauli, \& Wieser, 2016; Steinberg et al., 2012). To date, 
studies investigating the prioritization visual selective attention driven by reward mainly focused on monetary reward (e.g., Bourgeois, Chelazzi, \& Vuilleumier, 2016; Chelazzi et al., 2013; Hickey et al., 2010; Krebs et al., 2011). However, alternatives for monetary reward are conceivable (O'Doherty, 2004). It was argued that both monetary and social reward (e.g., caresses, social approval from others) activate comparable brain areas (Izuma, Saito, \& Sadato, 2008; Lin et al., 2012; Saxe \& Haushofer, 2008). However, as monetary and social reward are ranked among secondary rewards, using primary rewards (food or liquid; Schultz, 2006; Seitz, Kim, \& Watanabe, 2009) might provide a closer link to reward effects reported in animal studies as it has been suggested that neural activations might be reward type-dependent (for a review, see Sescousse, Caldú, Segura, \& Dreher, 2013).

In addition to ERPs, additional psychophysiological measures were collected. Pupillometric data in Study 2 revealed, that the explicit knowledge about the outcome-indicating cues at the beginning of the trial was strongly modulating the subsequent pupil dilation with an increased pupil size for reward- and loss- compared to zero outcome-indicating cues during the learning session. In contrast to ERP data, the expectancy of potential reward and loss seems to comparably impact physiological arousal. During delayed testing, modulations of pupil dilations were largely absent, indicating that the direct information about potential gain or loss is mandatory to arouse participants. Although, the results of Study 2 indicate that pupil dilations are an excellent measure to investigate the direct impacts of a motivational context, they do not seem to be sensitive to associated motivational salience which needs to be confirmed in future research. In Study 3, zygomaticus major activity was recorded in addition to ERPs as Sims et al. (2012) demonstrated that previously reward-associated neutral faces later presented with a happy expression led to a stronger zygomaticus activity than a happy face without previous reward association. However, neither effects of happy facial expressions and reward associations nor their integration were revealed. Therefore, future research is mandatory to investigate whether measuring zygomaticus major activity is useful to investigate effects of associated motivational salience in associative learning paradigms. In addition, it could be the subject of future research, whether corrugator supercilii activity might be susceptible to loss associations or potentially reduced by reward associations.

\section{Conclusion}

The aim of the present $\mathrm{PhD}$ project was the dissociation of impacts of associated motivational and inherent emotional salience on human face processing. In three studies, it was demonstrated that inherently neutral facial expressions gain salience via explicit and implicit associative learning and that their neural processing is comparable to the processing of emotional expressions. Reward associated neutral faces and angry facial expressions impacted the perceptual processing (P1) and/or the higher-order evaluations (LPC component). These findings indicate a distinct processing similarity of reward and threat, as they were suggested to be equally relevant to assure well-being and survival. However, EPN modulations driven by associated motivational salience were absent in all three studies, whereas the EPN was consistently impacted by inherently happy and angry expressions. 
This might suggest that there are also processing differences, as the EPN was referred to be an automatic indicator of emotional meaning independent of task demands. In addition, evidence revealed that reward elicited a prioritized processing compared to loss, even when the experimental design assured an equalization of outcomes. To sum up, results of the present studies confirmed that emotionally and motivationally relevant stimuli elicit a comparable processing advantage, as proposed by the value-driven attention mechanism (B. A. Anderson, 2013), however, associated motivational salience does not seem to trigger an inherent emotional meaning. 


\section{References}

Adolphs, R. (2003). Cognitive neuroscience of human social behaviour. Nature Reviews Neuroscience, 4(3), 165-178.

Aguado, L., Valdés-Conroy, B., Rodríguez, S., Román, F. J., Diéguez-Risco, T., \& Fernández-Cahill, M. (2012). Modulation of early perceptual processing by emotional expression and acquired valence of faces. Journal of Psychophysiology, 26(1), $29-41$.

Alger, S. E., \& Payne, J. D. (2016). The differential effects of emotional salience on direct associative and relational memory during a nap. Cognitive, Affective, \& Behavioral Neuroscience, 16(6), 1150-1163.

Anderson, A. K. (2005). Affective influences on the attentional dynamics supporting awareness. Journal of Experimental Psychology: General, 134 (2), 258-281.

Anderson, B. A. (2013). A value-driven mechanism of attentional selection. Journal of Vision, 13(3), 1-16.

Anderson, B. A., Laurent, P. A., \& Yantis, S. (2014). Value-driven attentional priority signals in human basal ganglia and visual cortex. Brain Research, 1587, 88-96.

Awh, E., Belopolsky, A. V., \& Theeuwes, J. (2012). Top-down versus bottom-up attentional control: A failed theoretical dichotomy. Trends in Cognitive Sciences, 16(8), 437443.

Barbaro, L., Peelen, M. V., \& Hickey, C. (2017). Valence, not utility, underlies rewarddriven prioritization in human vision. Journal of Neuroscience, 37(43), 10438-10450.

Batterink, L., \& Neville, H. (2011). Implicit and explicit mechanisms of word learning in a narrative context: an event-related potential study. Journal of Cognitive Neuroscience, 23(11), 3181-3196.

Batty, M., \& Taylor, M. J. (2003). Early processing of the six basic facial emotional expressions. Cognitive Brain Research, 17(3), 613-620.

Bayer, M., Rossi, V., Vanlessen, N., Grass, A., Schacht, A., \& Pourtois, G. (2017). Independent effects of motivation and spatial attention in the human visual cortex. Social Cognitive and Affective Neuroscience, 12(1), 146-156.

Bayer, M., \& Schacht, A. (2014). Event-related brain responses to emotional words, pictures, and faces-a cross-domain comparison. Frontiers in Psychology, 5(1106), $1-10$.

Bayer, M., Sommer, W., \& Schacht, A. (2012). P1 and beyond: functional separation of multiple emotion effects in word recognition. Psychophysiology, 49(7), 959-969.

Bentin, S., Allison, T., Puce, A., Perez, E., \& McCarthy, G. (1996). Electrophysiological 
studies of face perception in humans. Journal of Cognitive Neuroscience, 8(6), 551565 .

Berridge, C. W., \& Waterhouse, B. D. (2003). The locus coeruleus-noradrenergic system: modulation of behavioral state and state-dependent cognitive processes. Brain Research Reviews, 42(1), 33-84.

Berry, D., \& Dienes, Z. P. (1993). Implicit learning: Theoretical and empirical issues. Hove, UK.: Erlbaum.

Bhanji, J. P., \& Delgado, M. R. (2014). The social brain and reward: social information processing in the human striatum. Wiley Interdisciplinary Reviews: Cognitive Science, 5(1), 61-73.

Bourgeois, A., Chelazzi, L., \& Vuilleumier, P. (2016). How motivation and reward learning modulate selective attention. Progress in Brain Research, 229, 325-342.

Bourgeois, A., Neveu, R., \& Vuilleumier, P. (2016). How does awareness modulate goaldirected and stimulus-driven shifts of attention triggered by value learning? PloS One, $11(8)$, e0160469.

Bradley, M. M., Miccoli, L., Escrig, M. A., \& Lang, P. J. (2008). The pupil as a measure of emotional arousal and autonomic activation. Psychophysiology, 45(4), 602-607.

Bradley, M. M., Sabatinelli, D., Lang, P. J., Fitzsimmons, J. R., King, W., \& Desai, P. (2003). Activation of the visual cortex in motivated attention. Behavioral Neuroscience, $117(2), 369-380$.

Bruce, V., \& Young, A. (1986). Understanding face recognition. British Journal of Psychology, $77(3), 305-327$.

Brunyé, T. T., \& Gardony, A. L. (2017). Eye tracking measures of uncertainty during perceptual decision making. International Journal of Psychophysiology, 120, 60-68.

Bublatzky, F., Gerdes, A. B., White, A. J., Riemer, M., \& Alpers, G. W. (2014). Social and emotional relevance in face processing: happy faces of future interaction partners enhance the late positive potential. Frontiers in Human Neuroscience, 8(493), 1-10.

Calvo, M. G., \& Beltrán, D. (2013). Recognition advantage of happy faces: tracing the neurocognitive processes. Neuropsychologia, 51 (11), 2051-2061.

Calvo, M. G., \& Lundqvist, D. (2008). Facial expressions of emotion (KDEF): Identification under different display-duration conditions. Behavior Research Methods, 40(1), 109115.

Chapman, C. S., Gallivan, J. P., Wong, J. D., Wispinski, N. J., \& Enns, J. T. (2015). The snooze of lose: Rapid reaching reveals that losses are processed more slowly than gains. Journal of Experimental Psychology: General, 144(4), 844-863.

Chelazzi, L., Perlato, A., Santandrea, E., \& Della Libera, C. (2013). Rewards teach visual selective attention. Vision Research, 85, 58-72.

Choi, J. M., Padmala, S., \& Pessoa, L. (2015). Counteracting effect of threat on reward enhancements during working memory. Cognition and Emotion, 29(8), 1517-1526.

Choi, J. M., Padmala, S., Spechler, P., \& Pessoa, L. (2014). Pervasive competition between threat and reward in the brain. Social Cognitive and Affective Neuroscience, 9(6), $737-750$. 
Cleeremans, A., Destrebecqz, A., \& Boyer, M. (1998). Implicit learning: News from the front. Trends in Cognitive Sciences, 2(10), 406-416.

Connor, C. E., Egeth, H. E., \& Yantis, S. (2004). Visual attention: bottom-up versus top-down. Current Biology, 14 (19), R850-R852.

Corbetta, M., \& Shulman, G. L. (2002). Control of goal-directed and stimulus-driven attention in the brain. Nature Reviews Neuroscience, 3(3), 201-215.

Crouzet, S. M., Kirchner, H., \& Thorpe, S. J. (2010). Fast saccades toward faces: face detection in just $100 \mathrm{~ms}$. Journal of Vision, 10(4), 1-17.

Cuthbert, B. N., Schupp, H. T., Bradley, M. M., Birbaumer, N., \& Lang, P. J. (2000). Brain potentials in affective picture processing: covariation with autonomic arousal and affective report. Biological Psychology, 52(2), 95-111.

Della Libera, C., \& Chelazzi, L. (2006). Visual selective attention and the effects of monetary rewards. Psychological Science, 17(3), 222-227.

Della Libera, C., \& Chelazzi, L. (2009). Learning to attend and to ignore is a matter of gains and losses. Psychological Science, 20(6), 778-784.

Dickinson, A. (1981). Conditioning and associative learning. British Medical Bulletin, $37(2), 165-168$.

Dimberg, U. (1982). Facial reactions to facial expressions. Psychophysiology, 19(6), 643647.

Dimberg, U., \& Petterson, M. (2000). Facial reactions to happy and angry facial expressions: Evidence for right hemisphere dominance. Psychophysiology, 37(5), 693-696.

Di Russo, F., Martínez, A., \& Hillyard, S. A. (2003). Source analysis of event-related cortical activity during visuo-spatial attention. Cerebral Cortex, 13(5), 486-499.

Di Russo, F., Martínez, A., Sereno, M. I., Pitzalis, S., \& Hillyard, S. A. (2001). Cortical sources of the early components of the visual evoked potential. Human Brain Mapping, 15(2), 95-111.

Dunsmoor, J. E., Murty, V. P., Davachi, L., \& Phelps, E. A. (2015). Emotional learning selectively and retroactively strengthens memories for related events. Nature, 520 (7547), 345-348.

Eastwood, J. D., Smilek, D., \& Merikle, P. M. (2001). Differential attentional guidance by unattended faces expressing positive and negative emotion. Attention, Perception, $\mathcal{E}$ Psychophysics, 63(6), 1004-1013.

Eimer, M., Holmes, A., \& McGlone, F. P. (2003). The role of spatial attention in the processing of facial expression: an ERP study of rapid brain responses to six basic emotions. Cognitive, Affective, \& Behavioral Neuroscience, 3(2), 97-110.

Einhäuser, W., Stout, J., Koch, C., \& Carter, O. (2008). Pupil dilation reflects perceptual selection and predicts subsequent stability in perceptual rivalry. Proceedings of the National Academy of Sciences, 105(5), 1704-1709.

Ekman, P. (1992). An argument for basic emotions. Cognition $\&$ Emotion, 6(3-4), 169200.

Eppinger, B., Hämmerer, D., \& Li, S.-C. (2011). Neuromodulation of reward-based learning and decision making in human aging. Annals of the New York Academy of Sciences, 
$1235(1), 1-17$.

Failing, M., \& Theeuwes, J. (2017). Selection history: how reward modulates selectivity of visual attention. Psychonomic Bulletin \& Review, 1-25.

Fecteau, J. H., \& Munoz, D. P. (2006). Salience, relevance, and firing: a priority map for target selection. Trends in Cognitive Sciences, 10(8), 382-390.

Fedota, J. R., McDonald, C. G., Roberts, D. M., \& Parasuraman, R. (2012). Contextual task difficulty modulates stimulus discrimination: electrophysiological evidence for interaction between sensory and executive processes. Psychophysiology, 49(10), $1384-1393$.

Fridlund, A. J., \& Cacioppo, J. T. (1986). Guidelines for human electromyographic research. Psychophysiology, 23(5), 567-589.

Frith, C. (2009). Role of facial expressions in social interactions. Philosophical Transactions of the Royal Society of London B: Biological Sciences, 364 (1535), 3453-3458.

Gamond, L., George, N., Lemaréchal, J.-D., Hugueville, L., Adam, C., \& Tallon-Baudry, C. (2011). Early influence of prior experience on face perception. NeuroImage, 54 (2), $1415-1426$.

Gehring, W. J., \& Willoughby, A. R. (2002). The medial frontal cortex and the rapid processing of monetary gains and losses. Science, 295(5563), 2279-2282.

Gilzenrat, M. S., Nieuwenhuis, S., Jepma, M., \& Cohen, J. D. (2010). Pupil diameter tracks changes in control state predicted by the adaptive gain theory of locus coeruleus function. Cognitive, Affective, \& Behavioral Neuroscience, 10(2), 252-269.

Grandjean, D., Sander, D., \& Scherer, K. R. (2008). Conscious emotional experience emerges as a function of multilevel, appraisal-driven response synchronization. Consciousness and Cognition, 17(2), 484-495.

Hajcak, G., Moser, J. S., Holroyd, C. B., \& Simons, R. F. (2007). It's worse than you thought: The feedback negativity and violations of reward prediction in gambling tasks. Psychophysiology, 44(6), 905-912.

Hammerschmidt, W., Kagan, I., Kulke, L., \& Schacht, A. (2017). Implicit reward associations impact face processing: Time-resolved evidence from event-related brain potentials and pupil dilations. bioRxiv, 232538. doi: https://doi.org/10.1101/232538

Hammerschmidt, W., Sennhenn-Reulen, H., \& Schacht, A. (2017). Associated motivational salience impacts early sensory processing of human faces. NeuroImage, 156, 466-474.

Heydari, S., \& Holroyd, C. B. (2016). Reward positivity: reward prediction error or salience prediction error? Psychophysiology, 53(8), 1185-1192.

Hickey, C., Chelazzi, L., \& Theeuwes, J. (2010). Reward changes salience in human vision via the anterior cingulate. Journal of Neuroscience, 30(33), 11096-11103.

Hinojosa, J. A., Mercado, F., \& Carretié, L. (2015). N170 sensitivity to facial expression: a meta-analysis. Neuroscience 83 Biobehavioral Reviews, 55, 498-509.

Hintze, P., Junghöfer, M., \& Bruchmann, M. (2014). Evidence for rapid prefrontal emotional evaluation from visual evoked responses to conditioned gratings. Biological Psychology, 99, 125-136.

Holmes, A., Nielsen, M. K., Tipper, S., \& Green, S. (2009). An electrophysiological 
investigation into the automaticity of emotional face processing in high versus low trait anxious individuals. Cognitive, Affective, \& Behavioral Neuroscience, 9(3), 323-334.

Hu, P., Stylos-Allan, M., \& Walker, M. P. (2006). Sleep facilitates consolidation of emotional declarative memory. Psychological Science, 17(10), 891-898.

Ille, N., Berg, P., \& Scherg, M. (2002). Artifact correction of the ongoing EEG using spatial filters based on artifact and brain signal topographies. Journal of Clinical Neurophysiology, 19(2), 113-124.

Izuma, K., Saito, D. N., \& Sadato, N. (2008). Processing of social and monetary rewards in the human striatum. Neuron, 58(2), 284-294.

Javadi, A. H., Walsh, V., \& Lewis, P. A. (2011). Offline consolidation of procedural skill learning is enhanced by negative emotional content. Experimental Brain Research, $208(4), 507-517$.

Junghöfer, M., Bradley, M. M., Elbert, T. R., \& Lang, P. J. (2001). Fleeting images: a new look at early emotion discrimination. Psychophysiology, 38(2), 175-178.

Kahneman, D. (1973). Attention and effort (Vol. 1063). Prentice-Hall Englewood Cliffs, NJ.

Kahneman, D., \& Tversky, A. (1979). Prospect theory: an analysis of decisions under risk. Econometrica, 47(2), 263-291.

Kaltwasser, L., Ries, S., Sommer, W., Knight, R. T., \& Willems, R. M. (2013). Independence of valence and reward in emotional word processing: electrophysiological evidence. Frontiers in Psychology, 4 (168), 1-15.

Kang, O. E., Huffer, K. E., \& Wheatley, T. P. (2014). Pupil dilation dynamics track attention to high-level information. PloS One, 9(8), e102463.

Kastner, A. K., Flohr, E. L., Pauli, P., \& Wieser, M. J. (2016). A scent of anxiety: Olfactory context conditioning and its influence on social cues. Chemical Senses, 41(2), 143-153.

Kastner, A. K., Pauli, P., \& Wieser, M. J. (2015). Sustained attention in context conditioning: evidence from steady-state VEPs. International Journal of Psychophysiology, $98(3), 546-556$.

Keil, A., Bradley, M. M., Hauk, O., Rockstroh, B., Elbert, T., \& Lang, P. J. (2002). Large-scale neural correlates of affective picture processing. Psychophysiology, 39(5), 641-649.

Kim, S. H., Yoon, H. S., Kim, H., \& Hamann, S. (2015). Individual differences in sensitivity to reward and punishment and neural activity during reward and avoidance learning. Social Cognitive and Affective Neuroscience, 10(9), 1219-1227.

Kirita, T., \& Endo, M. (1995). Happy face advantage in recognizing facial expressions. Acta Psychologica, 89(2), 149-163.

Kiss, M., Van Velzen, J., \& Eimer, M. (2008). The N2pc component and its links to attention shifts and spatially selective visual processing. Psychophysiology, 45(2), 240-249.

Kissler, J., Herbert, C., Peyk, P., \& Junghofer, M. (2007). Buzzwords: early cortical 
responses to emotional words during reading. Psychological Science, 18(6), 475480.

Kissler, J., Herbert, C., Winkler, I., \& Junghofer, M. (2009). Emotion and attention in visual word processing - An ERP study. Biological Psychology, 80(1), 75-83.

Krebs, R. M., Boehler, C. N., Egner, T., \& Woldorff, M. G. (2011). The neural underpinnings of how reward associations can both guide and misguide attention. Journal of Neuroscience, 31(26), 9752-9759.

Krebs, R. M., \& Woldorff, M. G. (2017). Cognitive control and reward. In T. Egner (Ed.), The Wiley Handbook of Cognitive Control (1st ed., pp. 422-439). John Wiley and Sons, Ltd., Chichester (UK).

Kret, M. E., Roelofs, K., Stekelenburg, J. J., \& de Gelder, B. (2013). Emotional signals from faces, bodies and scenes influence observers' face expressions, fixations and pupil-size. Frontiers in Human Neuroscience, 7(810), 1-9.

Krishnamoorthy, K., Thomson, J., \& Cai, Y. (2004). An exact method of testing equality of several binomial proportions to a specified standard. Computational Statistics 8 Data Analysis, 45(4), 697-707.

Kulke, L., Bayer, M., Grimm, A.-M., \& Schacht, A. (2017). Differential effects of learned associations with words and pseudowords on event-related brain potentials. bioRxiv, 240945. doi: http://dx.doi.org/10.1101/240945

Laeng, B., Sirois, S., \& Gredebäck, G. (2012). Pupillometry: a window to the preconscious? Perspectives on Psychological Science, 7(1), 18-27.

Lang, P. J. (2010). Emotion and motivation: Toward consensus definitions and a common research purpose. Emotion Review, 2(3), 229-233.

Lang, P. J., \& Bradley, M. M. (2010). Emotion and the motivational brain. Biological Psychology, 84(3), 437-450.

Lang, P. J., Bradley, M. M., \& Cuthbert, B. N. (1997). International affective picture system (IAPS): Technical manual and affective ratings. NIMH Center for the Study of Emotion and Attention, 39-58.

Lang, P. J., Bradley, M. M., Cuthbert, B. N., et al. (1997). Motivated attention: Affect, activation, and action. In P. Lang, J. R. Simon, \& R. T. Balaban (Eds.), Attention and orienting: Sensory and motivational processes. Hillsdale, NJ: Erlbaum.

Langton, S. R., Law, A. S., Burton, A. M., \& Schweinberger, S. R. (2008). Attention capture by faces. Cognition, 107(1), 330-342.

Larsen, J. T., Norris, C. J., \& Cacioppo, J. T. (2003). Effects of positive and negative affect on electromyographic activity over zygomaticus major and corrugator supercilii. Psychophysiology, 40(5), 776-785.

Leppänen, J. M., \& Hietanen, J. K. (2004). Positive facial expressions are recognized faster than negative facial expressions, but why? Psychological Research, 69(1-2), 22-29.

Lin, A., Adolphs, R., \& Rangel, A. (2012). Social and monetary reward learning engage overlapping neural substrates. Social Cognitive and Affective Neuroscience, 7(3), 274-281.

Liu, H.-H., Hsieh, M. H., Hsu, Y.-F., \& Lai, W.-S. (2015). Effects of affective arousal on 
choice behavior, reward prediction errors, and feedback-related negativities in human reward-based decision making. Frontiers in Psychology, 6(592), 1-14.

Luck, S. J. (2014). An introduction to the event-related potential technique. Cambridge, MA, MIT Press.

Lundqvist, D., Flykt, A., \& Öhman, A. (1998). The Karolinska directed emotional faces - KDEF. CD ROM from Department of Clinical Neuroscience, Psychology section, Karolinska Institutet, Stockholm (Sweden). (ISBN: 91-630-7164-9)

Mackintosh, N. J. (1983). Conditioning and associative learning. Clarendon Press Oxford.

Marinkovic, K., \& Halgren, E. (1998). Human brain potentials related to the emotional expression, repetition, and gender of faces. Psychobiology, 26 (4), 348-356.

Massar, S. A., Lim, J., Sasmita, K., \& Chee, M. W. (2016). Rewards boost sustained attention through higher effort: A value-based decision making approach. Biological Psychology, 120, 21-27.

Mathôt, S., Siebold, A., Donk, M., \& Vitu, F. (2015). Large pupils predict goal-driven eye movements. Journal of Experimental Psychology: General, 144(3), 513-521.

McTeague, L. M., Gruss, L. F., \& Keil, A. (2015). Aversive learning shapes neuronal orientation tuning in human visual cortex. Nature Communications, 6, 1-8.

Meadows, C. C., Gable, P. A., Lohse, K. R., \& Miller, M. W. (2016). The effects of reward magnitude on reward processing: An averaged and single trial event-related potential study. Biological Psychology, 118, 154-160.

Mehu, M. (2014). An evolutionary perspective on facial behavior. In C. Müller, A. Cienki, E. Fricke, S. Ladewig, D. McNeill, \& J. Bressem (Eds.), Body - Language - Communication: Multimodal perspectives on language in communication (pp. 1962-1968). Berlin (Germany): De Gruyter Mouton.

Morel, S., Beaucousin, V., Perrin, M., \& George, N. (2012). Very early modulation of brain responses to neutral faces by a single prior association with an emotional context: evidence from MEG. NeuroImage, 61(4), 1461-1470.

Mueller, E. M., Panitz, C., Hermann, C., \& Pizzagalli, D. A. (2014). Prefrontal oscillations during recall of conditioned and extinguished fear in humans. Journal of Neuroscience, 34(21), 7059-7066.

Mueller, E. M., \& Pizzagalli, D. A. (2016). One-year-old fear memories rapidly activate human fusiform gyrus. Social Cognitive and Affective Neuroscience, 11(2), 308-316.

Murphy, P. R., O'Connell, R. G., O’Sullivan, M., Robertson, I. H., \& Balsters, J. H. (2014). Pupil diameter covaries with bold activity in human locus coeruleus. Human Brain Mapping, 35(8), 4140-4154.

Nieuwenhuis, S., Aston-Jones, G., \& Cohen, J. D. (2005). Decision making, the P3, and the locus coeruleus-norepinephrine system. Psychological Bulletin, 131(4), 510-532.

Nikitin, J., \& Freund, A. (2010). A motivational perspective on reactions to emotional faces. In A. Freitas-Magalhães (Ed.), Emotional expression: The brain and the face (Vol. 2, pp. 85-108). Oporto (Portugal): University Fernando Pessoa Press.

Öhman, A. (1986). Face the beast and fear the face: animal and social fears as prototypes for evolutionary analyses of emotion. Psychophysiology, 23(2), 123-145. 
Öhman, A., \& Mineka, S. (2001). Fears, phobias, and preparedness: toward an evolved module of fear and fear learning. Psychological Review, 108(3), 483-522.

Oldfield, R. (1971). The assessment and analysis of handedness: the Edinburgh inventory. Neuropsychologia, 9(1), 97-113.

Olofsson, J. K., Nordin, S., Sequeira, H., \& Polich, J. (2008). Affective picture processing: an integrative review of ERP findings. Biological Psychology, 77(3), 247-265.

O'Doherty, J. P. (2004). Reward representations and reward-related learning in the human brain: insights from neuroimaging. Current Opinion in Neurobiology, 14(6), 769776.

Palazova, M. (2014). Where are emotions in words? Functional localization of valence effects in visual word recognition. Frontiers in Psychology, 5(1105), 1-3.

Partala, T., \& Surakka, V. (2003). Pupil size variation as an indication of affective processing. International Journal of Human-Computer Studies, 59(1), 185-198.

Paulus, A., \& Wentura, D. (2014). Threatening joy: Approach and avoidance reactions to emotions are influenced by the group membership of the expresser. Cognition $\& 5$ Emotion, 28(4), 656-677.

Paulus, A., \& Wentura, D. (2016). It depends: Approach and avoidance reactions to emotional expressions are influenced by the contrast emotions presented in the task. Journal of Experimental Psychology: Human Perception and Performance, 42(2), $197-212$.

Pavlov, I. P. (1927). Conditional reflexes: An investigation of the physiological activity of the cerebral cortex.

Pessoa, L. (2008). On the relationship between emotion and cognition. Nature Reviews Neuroscience, 9(2), 148-158.

Pessoa, L. (2015a). Multiple influences of reward on perception and attention. Visual Cognition, 23(1-2), 272-290.

Pessoa, L. (2015b). Précis on the cognitive-emotional brain. Behavioral and Brain Sciences, $38,1-66$.

Pessoa, L., \& Engelmann, J. B. (2010). Embedding reward signals into perception and cognition. Frontiers in Neuroscience, 4 (17), 1-8.

Peysakhovich, V., Causse, M., Scannella, S., \& Dehais, F. (2015). Frequency analysis of a task-evoked pupillary response: luminance-independent measure of mental effort. International Journal of Psychophysiology, 97(1), 30-37.

Pfabigan, D. M., Alexopoulos, J., Bauer, H., \& Sailer, U. (2011). Manipulation of feedback expectancy and valence induces negative and positive reward prediction error signals manifest in event-related brain potentials. Psychophysiology, 48(5), 656-664.

Pfabigan, D. M., Sailer, U., \& Lamm, C. (2015). Size does matter! Perceptual stimulus properties affect event-related potentials during feedback processing. Psychophysiology, 52(9), 1238-1247.

Pivik, R. T., Broughton, R. J., Coppola, R., Davidson, R. J., Fox, N., \& Nuwer, M. R. (1993). Guidelines for the recording and quantitative analysis of electroencephalographic activity in research contexts. Psychophysiology, 30(6), 547-558. 
Posner, J., Russell, J. A., \& Peterson, B. S. (2005). The circumplex model of affect: An integrative approach to affective neuroscience, cognitive development, and psychopathology. Development and Psychopathology, 17(3), 715-734.

Pourtois, G., Grandjean, D., Sander, D., \& Vuilleumier, P. (2004). Electrophysiological correlates of rapid spatial orienting towards fearful faces. Cerebral Cortex, 14(6), 619-633.

Pourtois, G., Schettino, A., \& Vuilleumier, P. (2013). Brain mechanisms for emotional influences on perception and attention: what is magic and what is not. Biological Psychology, 92(3), 492-512.

Pratt, N., Willoughby, A., \& Swick, D. (2011). Effects of working memory load on visual selective attention: behavioral and electrophysiological evidence. Frontiers in Human Neuroscience, 5(57), 1-9.

Pulcu, E., \& Browning, M. (2017). Affective bias as a rational response to the statistics of rewards and punishments. eLife, 6, e27879.

Raymond, J. E., \& O'Brien, J. L. (2009). Selective visual attention and motivation: The consequences of value learning in an attentional blink task. Psychological Science, $20(8), 981-988$.

Recio, G., Schacht, A., \& Sommer, W. (2014). Recognizing dynamic facial expressions of emotion: Specificity and intensity effects in event-related brain potentials. Biological Psychology, 96, 111-125.

Recio, G., Sommer, W., \& Schacht, A. (2011). Electrophysiological correlates of perceiving and evaluating static and dynamic facial emotional expressions. Brain Research, $1376,66-75$.

Rehbein, M. A., Steinberg, C., Wessing, I., Pastor, M. C., Zwitserlood, P., Keuper, K., \& Junghöfer, M. (2014). Rapid plasticity in the prefrontal cortex during affective associative learning. PloS One, 9(10), e110720.

Rellecke, J., Palazova, M., Sommer, W., \& Schacht, A. (2011). On the automaticity of emotion processing in words and faces: event-related brain potentials evidence from a superficial task. Brain and Cognition, 77(1), 23-32.

Rellecke, J., Sommer, W., \& Schacht, A. (2012). Does processing of emotional facial expressions depend on intention? Time-resolved evidence from event-related brain potentials. Biological Psychology, 90(1), 23-32.

Rellecke, J., Sommer, W., \& Schacht, A. (2013). Emotion effects on the N170: a question of reference? Brain Topography, 26(1), 62-71.

Rolls, E. T. (2013). Emotion and decision making explained. Oxford University Press.

Rossi, V., Vanlessen, N., Bayer, M., Grass, A., Pourtois, G., \& Schacht, A. (2017). Motivational salience modulates early visual cortex responses across task sets. Journal of Cognitive Neuroscience, 29(6), 1-12.

Russell, J. A. (1980). A circumplex model of affect. Journal of Personality and Social Psychology, 39(6), 1161-1178.

Sailer, U., Fischmeister, F. P. S., \& Bauer, H. (2010). Effects of learning on feedbackrelated brain potentials in a decision-making task. Brain Research, 1342, 85-93. 
Sander, D., Grafman, J., \& Zalla, T. (2003). The human amygdala: an evolved system for relevance detection. Reviews in the Neurosciences, 14(4), 303-316.

San Martín, R. (2012). Event-related potential studies of outcome processing and feedbackguided learning. Frontiers in Human Neuroscience, 6(304), 1-17.

Santesso, D. L., Meuret, A. E., Hofmann, S. G., Mueller, E. M., Ratner, K. G., Roesch, E. B., \& Pizzagalli, D. A. (2008). Electrophysiological correlates of spatial orienting towards angry faces: a source localization study. Neuropsychologia, 46(5), 13381348.

Sato, A., Yasuda, A., Ohira, H., Miyawaki, K., Nishikawa, M., Kumano, H., \& Kuboki, T. (2005). Effects of value and reward magnitude on feedback negativity and P300. NeuroReport, 16(4), 407-411.

Sato, W., Kochiyama, T., Yoshikawa, S., \& Matsumura, M. (2001). Emotional expression boosts early visual processing of the face: ERP recording and its decomposition by independent component analysis. NeuroReport, 12(4), 709-714.

Satterthwaite, T. D., Green, L., Myerson, J., Parker, J., Ramaratnam, M., \& Buckner, R. L. (2007). Dissociable but inter-related systems of cognitive control and reward during decision making: evidence from pupillometry and event-related fMRI. NeuroImage, 37(3), 1017-1031.

Saxe, R., \& Haushofer, J. (2008). For love or money: a common neural currency for social and monetary reward. Neuron, 58(2), 164-165.

Schacht, A., Adler, N., Chen, P., Guo, T., \& Sommer, W. (2012). Association with positive outcome induces early effects in event-related brain potentials. Biological Psychology, $89(1), 130-136$.

Schacht, A., \& Sommer, W. (2009a). Emotions in word and face processing: early and late cortical responses. Brain and Cognition, 69(3), 538-550.

Schacht, A., \& Sommer, W. (2009b). Time course and task dependence of emotion effects in word processing. Cognitive, Affective, \& Behavioral Neuroscience, 9(1), 28-43.

Scherer, K. R. (2005). What are emotions? And how can they be measured? Social Science Information, 44(4), 695-729.

Scherer, K. R. (2009). The dynamic architecture of emotion: Evidence for the component process model. Cognition and Emotion, 23(7), 1307-1351.

Scherg, M. (2003). Artifacts: Using calibration data to generate artifact coefficients. Manual for BESA: Brain-Electrical Source Analysis software (Version 5.0). Munich, Germany: Megis Software GmbH.

Scheuthle, H., Carabias-Hütter, V., \& Kaiser, F. G. (2005). The motivational and instantaneous behavior effects of contexts: Steps toward a theory of goal-directed behavior. Journal of Applied Social Psychology, 35(10), 2076-2093.

Schultz, W. (2006). Behavioral theories and the neurophysiology of reward. Annual Review of Psychology, 57, 87-115.

Schupp, H. T., Markus, J., Weike, A. I., \& Hamm, A. O. (2003). Emotional facilitation of sensory processing in the visual cortex. Psychological Science, 14(1), 7-13.

Schupp, H. T., Öhman, A., Junghöfer, M., Weike, A. I., Stockburger, J., \& Hamm, A. O. 
(2004). The facilitated processing of threatening faces: an ERP analysis. Emotion, $4(2), 189-200$.

Seitz, A. R., Kim, D., \& Watanabe, T. (2009). Rewards evoke learning of unconsciously processed visual stimuli in adult humans. Neuron, 61(5), 700-707.

Sescousse, G., Caldú, X., Segura, B., \& Dreher, J.-C. (2013). Processing of primary and secondary rewards: a quantitative meta-analysis and review of human functional neuroimaging studies. Neuroscience \& Biobehavioral Reviews, 37(4), 681-696.

Sharot, T., Delgado, M. R., \& Phelps, E. A. (2004). How emotion enhances the feeling of remembering. Nature Neuroscience, 7(12), 1376-1380.

Sims, T. B., Van Reekum, C. M., Johnstone, T., \& Chakrabarti, B. (2012). How reward modulates mimicry: EMG evidence of greater facial mimicry of more rewarding happy faces. Psychophysiology, 49(7), 998-1004.

Skinner, B. (1953). Science and human behavior. The Macmillan Company, New York $(\mathrm{NY})$.

Skrandies, W. (1990). Global field power and topographic similarity. Brain Topography, 3(1), 137-141.

Smallwood, J., Brown, K. S., Tipper, C., Giesbrecht, B., Franklin, M. S., Mrazek, M. D., ... Schooler, J. W. (2011). Pupillometric evidence for the decoupling of attention from perceptual input during offline thought. PloS One, 6(3), e18298.

Steinberg, C., Dobel, C., Schupp, H. T., Kissler, J., Elling, L., Pantev, C., \& Junghöfer, M. (2012). Rapid and highly resolving: affective evaluation of olfactorily conditioned faces. Journal of Cognitive Neuroscience, 24(1), 17-27.

Steyer, R., Schwenkmezger, P., Notz, P., \& Eid, M. (1997). MDBF-Mehrdimensionaler Befindlichkeitsfragebogen. Göttingen, Germany: Hogrefe.

Stolarova, M., Keil, A., \& Moratti, S. (2006). Modulation of the C1 visual event-related component by conditioned stimuli: evidence for sensory plasticity in early affective perception. Cerebral Cortex, 16(6), 876-887.

Suess, F., Rabovsky, M., \& Abdel Rahman, R. (2014). Perceiving emotions in neutral faces: expression processing is biased by affective person knowledge. Social Cognitive and Affective Neuroscience, 10(4), 531-536.

Theeuwes, J. (2010). Top-down and bottom-up control of visual selection. Acta Psychologica, 135(2), 77-99.

Thorndike, E. L. (1927). The law of effect. The American Journal of Psychology, 39(1/4), $212-222$.

Tom, S. M., Fox, C. R., Trepel, C., \& Poldrack, R. A. (2007). The neural basis of loss aversion in decision-making under risk. Science, 315(5811), 515-518.

Trepel, C., Fox, C. R., \& Poldrack, R. A. (2005). Prospect theory on the brain? Toward a cognitive neuroscience of decision under risk. Cognitive Brain Research, 23(1), $34-50$.

Tversky, A., \& Kahneman, D. (1992). Advances in prospect theory: cumulative representation of uncertainty. Journal of Risk and Uncertainty, 5(4), 297-323.

Umemoto, A., HajiHosseini, A., Yates, M. E., \& Holroyd, C. B. (2017). Reward-based 
contextual learning supported by anterior cingulate cortex. Cognitive, Affective, $\mathfrak{G}$ Behavioral Neuroscience, 17(3), 642-651.

Unakafov, A. M. (2017). An exact test for equality of several binomial proportions to a specified standard, MATLAB Central File Exchange, retrieved Nov 08, 2017. https:// de.mathworks. com/matlabcentral/fileexchange/64987-an-exact-test-for -equality-of-several-binomial-proportions-to-a-specified-standard.

Urai, A. E., Braun, A., \& Donner, T. H. (2017). Pupil-linked arousal is driven by decision uncertainty and alters serial choice bias. Nature Communications, 8, 1-11.

Valdés-Conroy, B., Aguado, L., Fernández-Cahill, M., Romero-Ferreiro, V., \& DiéguezRisco, T. (2014). Following the time course of face gender and expression processing: a task-dependent ERP study. International Journal of Psychophysiology, 92(2), 5966.

Van Strien, J. W., De Sonneville, L. M., \& Franken, I. H. (2010). The late positive potential and explicit versus implicit processing of facial valence. NeuroReport, 21(9), 656661.

Ventura-Bort, C., Löw, A., Wendt, J., Dolcos, F., Hamm, A. O., \& Weymar, M. (2016). When neutral turns significant: brain dynamics of rapidly formed associations between neutral stimuli and emotional contexts. European Journal of Neuroscience, $44(5), 2176-2183$.

Vuilleumier, P. (2005). How brains beware: neural mechanisms of emotional attention. Trends in Cognitive Sciences, 9(12), 585-594.

Vuilleumier, P. (2015). Affective and motivational control of vision. Current Opinion in Neurology, 28(1), 29-35.

Vuilleumier, P., \& Pourtois, G. (2007). Distributed and interactive brain mechanisms during emotion face perception: evidence from functional neuroimaging. Neuropsychologia, 45 (1), 174-194.

Wächter, T., Lungu, O. V., Liu, T., Willingham, D. T., \& Ashe, J. (2009). Differential effect of reward and punishment on procedural learning. Journal of Neuroscience, $29(2), 436-443$.

Walentowska, W., Moors, A., Paul, K., \& Pourtois, G. (2016). Goal relevance influences performance monitoring at the level of the FRN and P3 components. Psychophysiology, 53(7), 1020-1033.

Walker, M. P. (2009). The role of sleep in cognition and emotion. Annals of the New York Academy of Sciences, 1156(1), 168-197.

Wei, P., \& Kang, G. (2014). Task relevance regulates the interaction between reward expectation and emotion. Experimental Brain Research, 232(6), 1783-1791.

Wei, P., Wang, D., \& Ji, L. (2016). Reward expectation regulates brain responses to task-relevant and task-irrelevant emotional words: ERP evidence. Social Cognitive and Affective Neuroscience, 11(2), 191-203.

Wieser, M. J., \& Brosch, T. (2012). Faces in context: a review and systematization of contextual influences on affective face processing. Frontiers in Psychology, 3(471), $1-13$. 
Wieser, M. J., Gerdes, A. B. M., Büngel, I., Schwarz, K. A., Mühlberger, A., \& Pauli, P. (2014). Not so harmless anymore: how context impacts the perception and electrocortical processing of neutral faces. NeuroImage, 92, 74-82.

Yao, S., Ding, C., Qi, S., \& Yang, D. (2014). Value associations of emotional faces can modify the anger superiority effect: behavioral and electrophysiological evidence. Social Cognitive and Affective Neuroscience, 9(6), 849-856.

Yeung, N., \& Sanfey, A. G. (2004). Independent coding of reward magnitude and valence in the human brain. Journal of Neuroscience, 24 (28), 6258-6264.

Zeelenberg, R., Wagenmakers, E.-J., \& Rotteveel, M. (2006). The impact of emotion on perception: Bias or enhanced processing? Psychological Science, 17(4), 287-291.

Zheng, Y., Li, Q., Zhang, Y., Shen, H., Gao, Q., \& Zhou, S. (2017). Reward processing in gain versus loss context: An ERP study. Psychophysiology, 54(7), 104-1053. 



\section{Acknowledgments}

First, I would like to thank Prof. Dr. Annekathrin Schacht. Her enthusiasm for research impressed and motivated me, especially in difficult situations. I could not have imagine having a better supervisor.

Furthermore, I would like to thank my thesis committee, Prof. Dr. Julia Fischer and Dr. Igor Kagan, for lively discussions and helpful advice throughout the last years.

I would particularly like to thank Adi Lausen for his time, patience and support especially in the last months, and for simply being the best office partner ever. Special thanks go to Simon Stephan and Dr. Louisa Kulke for their support in the last weeks of writing my dissertation.

I thank my ANaP Lab colleagues for the pleasant working atmosphere, the emotional encouragement and the fun we always had in the last years.

In a personal note, I want to thank my family and friends for all the mental support during my PhD. Words cannot express how grateful I am. 